\title{
A Convergent Synthesis of Alternating Fluorene-p-Xylene Oligomers and Delineation of the (Silver) Cation-Induced Folding
}

\author{
Vincent J. Chebny and Rajendra Rathore* \\ Department of Chemistry, Marquette University, \\ P.O. Box 1881, Milwaukee, Wisconsin 53201-1881
}

General Experimental Section $\quad$ S2

Synthetic Details of Intermediates $\quad$ S3-S24

${ }^{1} \mathrm{H} /{ }^{13} \mathrm{C}$ NMR Spectra of Intermediates $\quad$ S25-S37

MALDI-TOF MS spectrum of cyclic oligomers B (Figure S1) S38

Comparison of ${ }^{1} \mathrm{H}$ NMR spectra of Z1-Z9 (Figure S2) $\quad$ S39

Comparison of ${ }^{13} \mathrm{C}_{\text {NMR spectra of } \mathbf{Z 1 - Z 9} \text { (Figure S3) }} \quad$ S39

Comparison of $\mathrm{Ag}^{+}$binding by $\mathbf{Z 2}, \mathbf{Z 4}, \mathbf{Z 6}, \mathbf{Z 8}$ (Figure S4) $\quad \mathrm{S} 40$

Calculated molecular structures of $\mathbf{Z 1}, \mathbf{Z 3}, \mathbf{Z 5}, \mathbf{Z 7}, \mathbf{Z 9}$ (Figure S5) S41

Calculated molecular structures of $\mathbf{Z 2}, \mathbf{Z 4}, \mathbf{Z 6}, \mathbf{Z 8}$ (Figure S6) S41

${ }^{1} \mathrm{H}$ NMR Titrations with $\mathrm{Ag}^{+}$(Figure S7-S14) S42-S52

X-Ray Structure data of $\mathbf{Z 1}$ S53-S56 


\section{Experimental Section}

Materials. Fluorene, iodomethane, $n$-Butylithium, $\alpha, \alpha$-dichloro- $p$-xylene, silver trifluoromethanesulfonate, ethyl-4-(bromomethyl)benzoate, potassium hydroxide, tetrabutylammoniumbromide, potassium tert-butoxide, thionyl chloride, lithium tetrahydroaluminate, chloroform, methanol, hexanes, tetrahydrofuran, toluene, and ethyl acetate were commercially available and were used as received unless otherwise specified.

Anhydrous tetrahydrofuran (THF) was prepared by refluxing the commercial tetrahydrofuran (Aldrich) over lithium tetrahydroaluminate under an argon atmosphere for 24 hours followed by distillation. It was stored under an argon atmosphere in a Schlenk flask equipped with a Teflon valve fitted with Viton O-rings. Dichloromethane (Aldrich) was repeatedly stirred with fresh aliquots of conc. sulfuric acid ( $\sim 10 \%$ by volume) until the acid layer remained colorless. After separation it was washed successively with water, aqueous sodium bicarbonate, water, and saturated aqueous sodium chloride and dried over anhydrous calcium chloride. The dichloromethane was distilled twice from $\mathrm{P}_{2} \mathrm{O}_{5}$ under an argon atmosphere and stored in a Schlenk flask equipped with a Teflon valve fitted with Viton O-rings. The hexanes and toluene were distilled from $\mathrm{P}_{2} \mathrm{O}_{5}$ under an argon atmosphere and then refluxed over calcium hydride ( $\sim 12 \mathrm{hrs})$. After distillation from $\mathrm{CaH}_{2}$, the solvents were stored in Schlenk flasks under argon atmosphere. 


\section{Synthesis of Cyclic Oligomer B (method P1).}

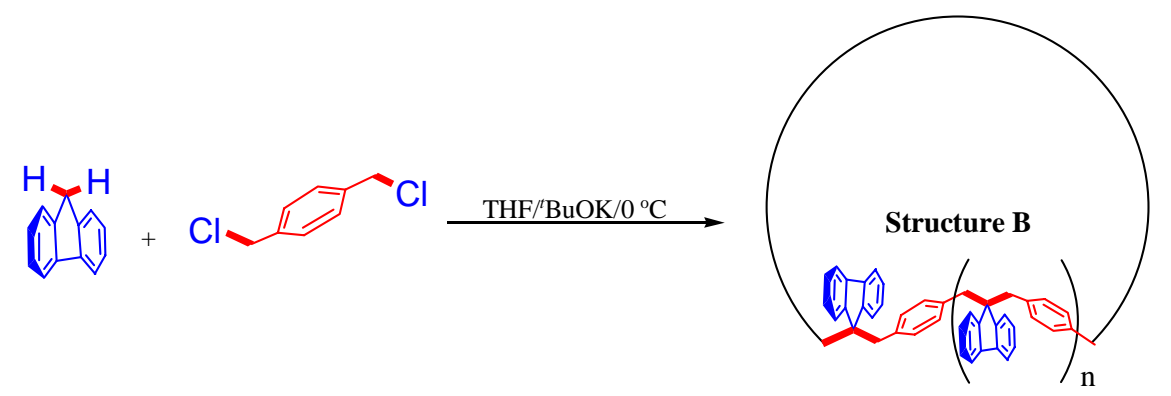

To a solution of fluorene $(0.17 \mathrm{~g}, 1.0 \mathrm{mmol})$ in anhydrous tetrahydrofuran $(20 \mathrm{ml})$ was added potassium tert-butoxide $(0.28 \mathrm{~g}, 2.5 \mathrm{mmol})$ at $\sim 0{ }^{\circ} \mathrm{C}$. After $10 \mathrm{~min}$. of stirring, $\alpha, \alpha-$ dichloro- $p$-xylene $(0.18 \mathrm{~g}, 1.0 \mathrm{mmol})$ was added and the mixture was stirred for $15 \mathrm{~min}$. The cooling bath was removed and the reaction mixture was stirred for an additional $12 \mathrm{~h}$. It was then poured onto water $(100 \mathrm{~mL})$ and the resulting mixture was extracted with dichloromethane (3 x $50 \mathrm{~mL}$ ). The combined dichloromethane extracts were dried over anhydrous magnesium sulfate, filtered, and evaporated under reduced pressure to afford a pale-yellow solid material. The crude material was purified by flash chromatography on silica gel using $5 \%$ ethyl acetate/hexanes as an eluent to afford a white solid. Yield: $0.3 \mathrm{~g}$; mp $270-272{ }^{\circ} \mathrm{C} ;{ }^{1} \mathrm{H}$ NMR $\left(\mathrm{CDCl}_{3}\right) \delta: 2.95(\mathrm{~s}, 4 \mathrm{H}), 6.21(\mathrm{~s}, 4 \mathrm{H}), 6.92(\mathrm{~d}, 2 \mathrm{H}), 7.18(\mathrm{~m}, 4 \mathrm{H}), 7.38(\mathrm{~d}, 2 \mathrm{H}) .{ }^{13} \mathrm{C}$ NMR

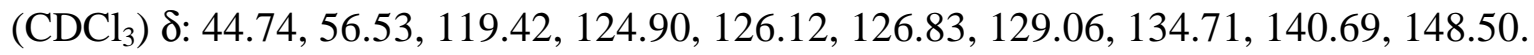

\section{Synthesis of Cyclic Oligomer B (method P2).}

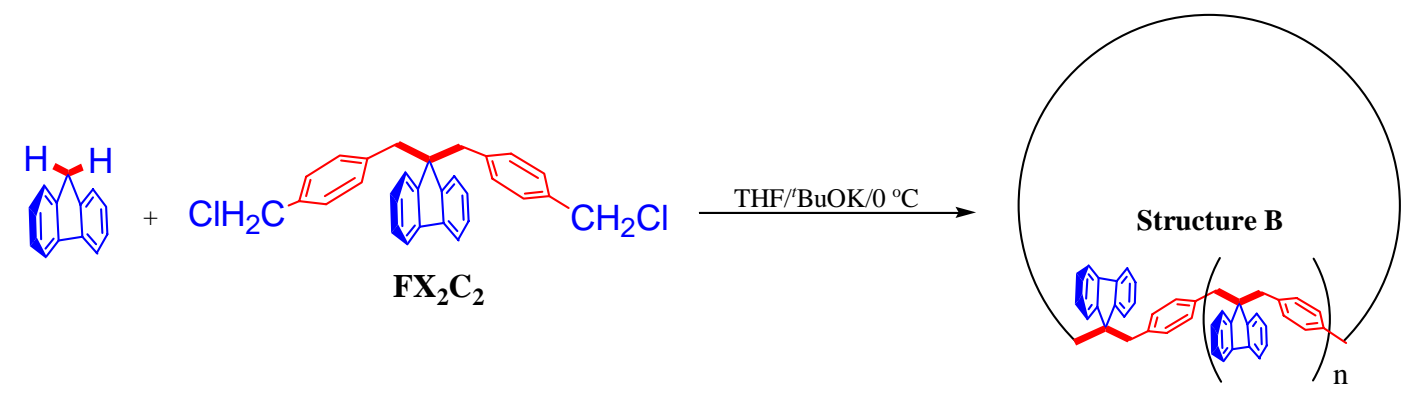

To a solution of fluorene $(0.16 \mathrm{~g}, 1.0 \mathrm{mmol})$ in anhydrous tetrahydrofuran $(20 \mathrm{ml})$ was added potassium tert-butoxide $(0.45 \mathrm{~g}, 4.0 \mathrm{mmol})$ to $\sim{ }^{\circ} \mathrm{C}$. After $10 \mathrm{~min}$. of stirring, $\alpha, \alpha-$ 
dichloro-fluorene derivative $\mathbf{F X}_{2} \mathbf{C}_{2}(0.44 \mathrm{~g}, 1.0 \mathrm{mmol})$ was added and the mixture was stirred for $15 \mathrm{~min}$. The cooling bath was removed and the reaction mixture was stirred for an additional 12 h. It was then poured onto water $(100 \mathrm{~mL})$ and the resulting mixture was extracted with dichloromethane $(3 \times 50 \mathrm{~mL})$. The combined dichloromethane extracts were dried over anhydrous magnesium sulfate, filtered, and evaporated under reduced pressure to afford a yellow solid material. The crude material was purified by flash chromatography on silica gel using $5 \%$ ethyl acetate / hexanes as an eluent to afford a white solid. Yield: $0.52 \mathrm{~g}$, with identical melting point and spectral data as listed above in method $\mathrm{P} 1$.

\section{Synthesis of Cyclic Oligomer B (method P3).}

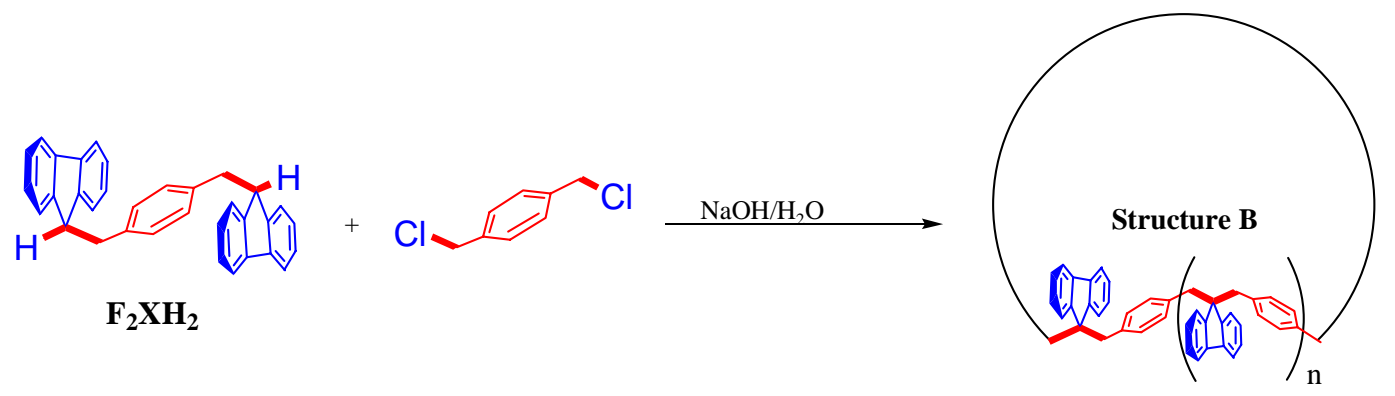

To a stirred solution of bifluorene derivative $\mathbf{F}_{2} \mathbf{X H}_{2}(2.0 \mathrm{~g}, 4.6 \mathrm{mmol})$ dissolved in toluene $(50 \mathrm{ml})$ was added successively $\mathrm{NaOH} / \mathrm{H}_{2} \mathrm{O}(50 \%$ by weight, $30 \mathrm{ml}), \alpha, \alpha$-dichloro- $p$ xylene $(0.70 \mathrm{~g}, 4.0 \mathrm{mmol})$, and tetra- $n$-butylammonium bromide $(0.3 \mathrm{~g})$ and the resulting mixture was heated and stirred vigorously at $\sim 50{ }^{\circ} \mathrm{C}$ for $12 \mathrm{~h}$. The reaction mixture was cooled to room temperature, poured onto ice-cold water $(100 \mathrm{~mL})$ and extracted with ethyl acetate $(3 \mathrm{x} 50 \mathrm{~mL})$. The combined ethyl acetate extracts were dried over anhydrous magnesium sulfate, filtered, and evaporated under reduced pressure to afford a yellow solid material. The crude material was purified by flash chromatography on silica gel using 5\% ethyl acetate/hexanes as an eluent to 
afford a pale yellow solid. Yield: $1.4 \mathrm{~g}$, with identical melting point and spectral data as listed above in method P1.

\section{Synthesis of Cyclic Oligomer B (method P4).}

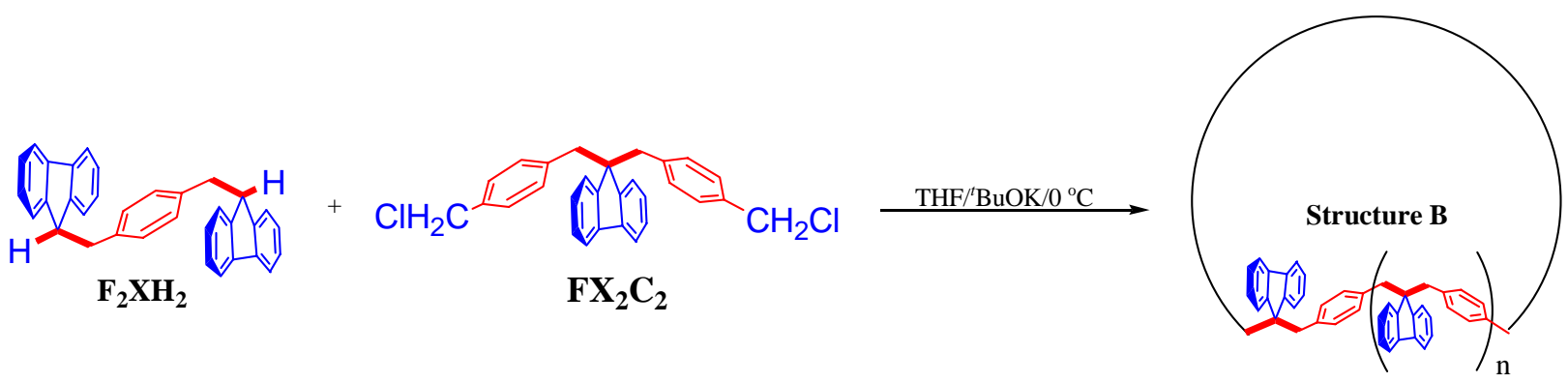

To a solution of difluorene derivative $\mathbf{F}_{2} \mathbf{X H}_{2}(0.43 \mathrm{~g}, 1.0 \mathrm{mmol})$ in anhydrous tetrahydrofuran $(20 \mathrm{ml})$ was added potassium tert-butoxide $(0.45 \mathrm{~g}, 4.0 \mathrm{mmol})$ at $\sim 0{ }^{\circ} \mathrm{C}$. After 10 min. of stirring, $\alpha, \alpha$-dichloro-fluorene derivative $\mathbf{F X}_{\mathbf{2}} \mathbf{C}_{\mathbf{2}}(0.44 \mathrm{~g}, 1.0 \mathrm{mmol})$ was added and the mixture was stirred for $15 \mathrm{~min}$. The cooling bath was removed and the resulting mixture was stirred for an additional $12 \mathrm{~h}$. It was then poured onto water $(100 \mathrm{~mL})$ and the aqueous layer was extracted with dichloromethane $(3 \times 50 \mathrm{~mL})$. The combined dichloromethane extracts were dried over anhydrous magnesium sulfate, filtered, and evaporated under reduced pressure to afford an orange solid material. The crude material was purified by flash chromatography on silica gel using 5\% ethyl acetate/hexanes as an eluent to afford a pale-yellow solid. Yield: $0.45 \mathrm{~g}$, with identical melting point and spectral data as listed above in method P1.

\section{Synthesis of Cyclic Oligomer B (method P5).}

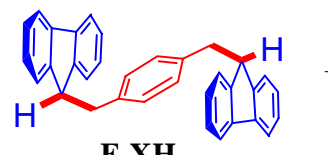

$\mathbf{F}_{2} \mathbf{X H}_{2}$

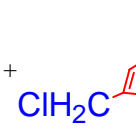

$\mathrm{ClH}_{2} \mathrm{C}$<smiles>CC12CC3CC(C1)CC(Cc1ccccc1)(C3)C2</smiles>
$\mathrm{F}_{2} \mathrm{X}_{3} \mathrm{C}_{2}$

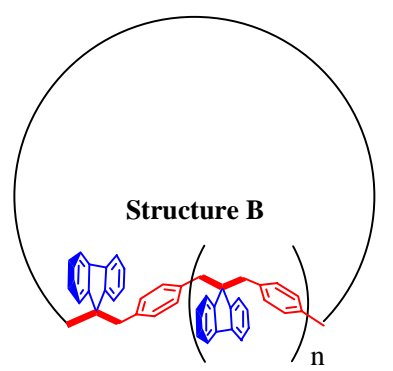


To a solution of bifluorene derivative $\mathbf{F}_{2} \mathbf{X H}_{2}(0.52 \mathrm{~g}, 1.2 \mathrm{mmol})$ in anhydrous tetrahydrofuran $(20 \mathrm{ml})$ was added potassium tert-butoxide $(0.45 \mathrm{~g}, 4.0 \mathrm{mmol})$ at $\sim 0{ }^{\circ} \mathrm{C}$. After 10 min. of stirring, $\alpha, \alpha$-dichloro-bifluorene derivative $\mathbf{F}_{2} \mathbf{X}_{3} \mathbf{C}_{2}(0.71 \mathrm{~g}, 1.0 \mathrm{mmol})$ was added and the mixture was stirred for $15 \mathrm{~min}$. The cooling bath was removed and the reaction mixture was stirred for an additional $12 \mathrm{~h}$. To the resulting solution iodomethane $(0.25 \mathrm{ml}, 4 \mathrm{mmol})$ was added and the reaction mixture stirred for another $2 \mathrm{~h}$. It was than poured onto water $(100 \mathrm{~mL})$ and the resulting mixture was extracted with dichloromethane $(3 \times 50 \mathrm{~mL})$. The combined dichloromethane extracts were dried over anhydrous magnesium sulfate, filtered, and evaporated under reduced pressure to afford an orange solid material. The crude material was purified by flash chromatography on silica gel using 5\% ethyl acetate/hexanes as an eluent to afford a paleyellow solid. Yield: $0.98 \mathrm{~g}$, with identical melting point and spectral data as listed above in method P1.

\section{Synthesis of FXEM via a One-Pot Reaction.}

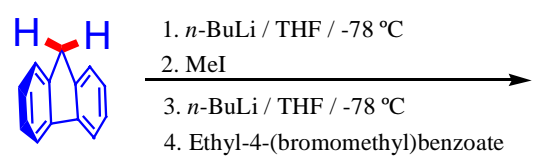

4. Ethyl-4-(bromomethyl)benzoate

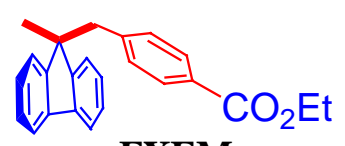

FXEM

To a pre-chilled $\left(-78{ }^{\circ} \mathrm{C}\right)$ solution of fluorene $(8.3 \mathrm{~g}, 50 \mathrm{mmol})$ in anhydrous tetrahydrofuran $(75 \mathrm{~mL})$ was added dropwise $n$-butyllithium $(2.5 \mathrm{M}$ in hexanes, $20 \mathrm{~mL})$ under an argon atmosphere. [Note that it is critical to maintain the temperature at $-78{ }^{\circ} \mathrm{C}$ throughout the reaction in order to avoid the formation of 9,9-dimethylfluorene.] After 10 min. of stirring at -78 ${ }^{\circ} \mathrm{C}$, iodomethane $(3.11 \mathrm{~mL}, 50 \mathrm{mmol})$ was added and the resulting mixture was stirred for 15 min. Any unreacted iodomethane which may have remained in the reaction mixture was removed in vacuo while warming the solution to room temperature. The resulting mixture was 
again cooled to $-78{ }^{\circ} \mathrm{C}$ and $n$-butyllithium $(2.5 \mathrm{M}$ solution in hexanes, $20 \mathrm{ml}$ ) was added dropwise to afford a dark-orange mixture which was stirred for $10 \mathrm{~min}$. To this orange-colored mixture was added solid ethyl-4-(bromomethyl)benzoate $(12.2 \mathrm{~g}, 50 \mathrm{mmol})$. The cooling bath was removed and the reaction mixture was stirred for $1 \mathrm{~h}$. It was then poured onto water (200 $\mathrm{mL})$ and the aqueous layer was extracted with dichloromethane $(3 \times 100 \mathrm{~mL})$. The combined dichloromethane extracts were dried over anhydrous magnesium sulfate, filtered, and evaporated under reduced pressure to afford a cream-colored solid. The crude solid was recrystallized from a mixture of $\mathrm{CH}_{2} \mathrm{Cl}_{2} / \mathrm{CH}_{3} \mathrm{OH}$ to afford a white crystalline solid. Yield: $15.0 \mathrm{~g}$ (88\%); mp 164-166 ${ }^{\circ} \mathrm{C} ;{ }^{1} \mathrm{H}$ NMR $\left(\mathrm{CDCl}_{3}\right) \delta: 1.31(\mathrm{t}, 3 \mathrm{H}), 1.61(\mathrm{~s}, 3 \mathrm{H}), 4.28(\mathrm{q}, 2 \mathrm{H}), 6.71(\mathrm{~d}, 2 \mathrm{H}), 7.29(\mathrm{~m}, 3 \mathrm{H}), 7.36$ (m, 3H), $7.56(\mathrm{~m}, 2 \mathrm{H}), 7.63(\mathrm{~d}, 2 \mathrm{H}) .{ }^{13} \mathrm{C} \mathrm{NMR}\left(\mathrm{CDCl}_{3}\right) \delta:$ 14.67, 25.90, 46.98, 51.75, 61.03, $120.13,123.64,127.04,127.36,128.22,128.56,130.19,140.06,143.06,150.62,166.80$.

\section{Synthesis of $\mathrm{FX}_{2} \mathrm{E}_{2}$ via a One-Pot Reaction}

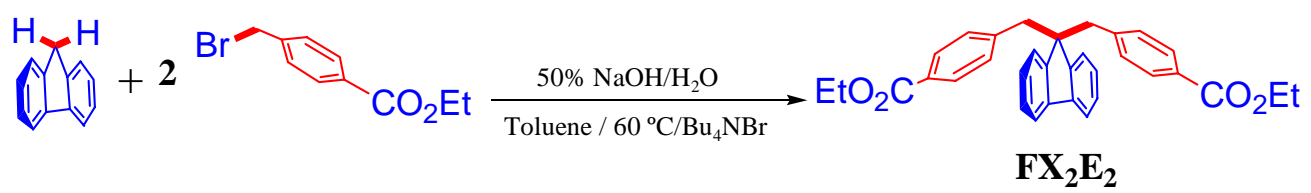

To a solution of fluorene $(1.33 \mathrm{~g}, 8 \mathrm{mmol})$, ethyl-4-(bromomethyl)benzoate $(5.0 \mathrm{~g}, 20$ mmol), and tetra- $n$-butylammonium bromide $(0.5 \mathrm{~g})$ in toluene $(25 \mathrm{ml})$ was added a solution of $\mathrm{NaOH} / \mathrm{H}_{2} \mathrm{O}$ (50\% by weight, $\left.25 \mathrm{ml}\right)$. The resulting mixture was heated and stirred vigorously at $60{ }^{\circ} \mathrm{C}$ and under an argon atmosphere for $12 \mathrm{~h}$. Resulting mixture was cooled to room temperature and the organic layer was separated. The aqueous layer was then extracted with ethyl acetate $(3 \times 100 \mathrm{ml})$, organic layers were combined, dried over anhydrous magnesium sulfate and evaporated under reduced pressure to obtain a yellow solid. The crude product was recrystallized with $\mathrm{CH}_{2} \mathrm{Cl}_{2} / \mathrm{CH}_{3} \mathrm{OH}$ to afford a white crystalline solid. Yield: $3.5 \mathrm{~g}$ (89\%); mp 
118-120 ${ }^{\circ} \mathrm{C} ;{ }^{1} \mathrm{H}$ NMR $\left(\mathrm{CDCl}_{3}\right) \delta: 1.31(\mathrm{t}, 6 \mathrm{H}), 3.45(\mathrm{~s}, 4 \mathrm{H}), 4.24(\mathrm{q}, 4 \mathrm{H}), 6.69(\mathrm{~d}, 4 \mathrm{H}), 7.28(\mathrm{~m}$, $8 \mathrm{H}), 7.43(\mathrm{~d}, 2 \mathrm{H}), 7.58(\mathrm{~d}, 2 \mathrm{H}) .{ }^{13} \mathrm{C} \mathrm{NMR}\left(\mathrm{CDCl}_{3}\right) \delta: 14.55,45.82,56.85,61.02,120.24$, $124.55,126.80,127.59,128.35,128.66,130.34,141.14,142.40,147.38,166.91$.

\section{Synthesis of $\mathbf{F}_{2} \mathrm{XH}_{2}$.}

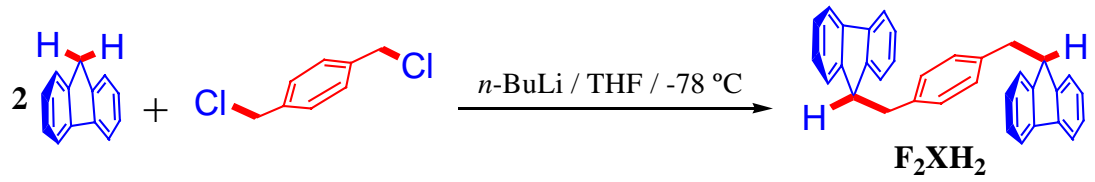

To a pre-chilled $\left(-78{ }^{\circ} \mathrm{C}\right)$ solution of fluorene $(8.0 \mathrm{~g}, 48 \mathrm{mmol})$ in anhydrous tetrahydrofuran $(100 \mathrm{~mL})$ under an argon atmosphere was added dropwise $n$-butyllithium $(2.5 \mathrm{M}$ in hexanes, $18 \mathrm{~mL}$ ) under an argon atmosphere to afford a red-orange solution. After $10 \mathrm{~min}$. of stirring, $\alpha, \alpha$-dichloro- $p$-xylene $(4.2 \mathrm{~g}, 24.0 \mathrm{mmol})$ was added and the mixture was stirred for 15 min. The cooling bath was removed and the reaction mixture was stirred for an additional $1 \mathrm{~h}$. It was then poured onto water $(100 \mathrm{~mL})$ and the resulting white precipitate was filtered and washed with ether (2 x $30 \mathrm{ml})$. Yield: $9.0 \mathrm{~g}(\sim 87 \%)$; mp 208-210 ${ }^{\circ} \mathrm{C} ;{ }^{1} \mathrm{H}$ NMR $\left(\mathrm{CDCl}_{3}\right) \delta: 3.22(\mathrm{~d}, 4 \mathrm{H})$, $4.39(\mathrm{t}, 2 \mathrm{H}), 7.01(\mathrm{~s}, 4 \mathrm{H}), 7.12(\mathrm{~m}, 8 \mathrm{H}), 7.29(\mathrm{t}, 4 \mathrm{H}), 7.68(\mathrm{~d}, 4 \mathrm{H}) ;{ }^{13} \mathrm{C} \mathrm{NMR}\left(\mathrm{CDCl}_{3}\right) \delta: 38.88$ $49.93,120.01,125.09,126.84,127.31,129.58,137.82,141.12,147.04$.

\section{Synthesis of $F_{2} X_{3} E_{2}$.}

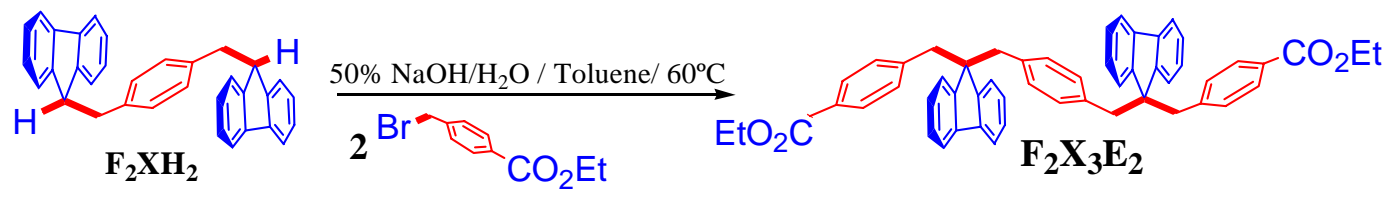

To a solution of $\mathbf{F}_{2} \mathbf{X H}_{2}$ (2.7 g, $6.2 \mathrm{mmol}$ ), ethyl-4-(bromomethyl)benzoate (2.7 g, 6.2 mmol), and tetra- $n$-butylammonium bromide $(0.5 \mathrm{~g})$ in toluene $(50 \mathrm{ml})$ was added $\mathrm{NaOH} / \mathrm{H}_{2} \mathrm{O}$ (50\% by weight, $30 \mathrm{ml}$ ), and the resulting mixture was stirred at $60{ }^{\circ} \mathrm{C}$ for $12 \mathrm{~h}$ under an argon 
atmosphere. Reaction mixture was then cooled to room temperature and the organic layer was separated from the aqueous layer. Aqueous layer was extracted with ethyl acetate $(3 \times 100 \mathrm{ml})$ and the combined organic extracts were dried over anhydrous magnesium sulfate and evaporated under reduced pressure to afford a yellow solid. The crude material was purified by flash chromatography on silica gel using $15 \%$ ethyl acetate/hexanes as eluent to afford a colorless solid. Yield: $3.1 \mathrm{~g} \mathrm{(79 \% );} \mathrm{mp} \mathrm{158-160}{ }^{\circ} \mathrm{C} ;{ }^{1} \mathrm{H}$ NMR $\left(\mathrm{CDCl}_{3}\right) \delta: 1.33(\mathrm{t}, 6 \mathrm{H}), 3.01(\mathrm{~s}, 4 \mathrm{H}), 3.14$ (s, $4 \mathrm{H}), 4.28(\mathrm{q}, 4 \mathrm{H}), 6.23(\mathrm{~s}, 4 \mathrm{H}), 6.51(\mathrm{~d}, 4 \mathrm{H}), 7.13(\mathrm{~m}, 12 \mathrm{H}), 7.31(\mathrm{~m}, 4 \mathrm{H}), 7.42(\mathrm{~d}, 4 \mathrm{H}) ;{ }^{13} \mathrm{C}$ $\operatorname{NMR}\left(\mathrm{CDCl}_{3}\right) \delta: 14.57,44.74,45.94,56.73,60.87,119.74,124.65,126.40,127.21,128.00$ $128.38,129.29,130.01,134.65,140.86,142.64,147.84,166.68$.

\section{Synthesis of FXAM.}

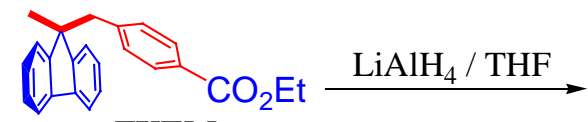

FXEM

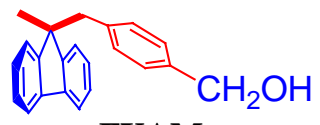

FXAM

To a solution of ester FXEM $(9.0 \mathrm{~g}, 26.0 \mathrm{mmol})$ in tetrahydrofuran $(75 \mathrm{~mL})$ under an argon atmosphere was added carefully lithium aluminum hydride $(1.4 \mathrm{~g}, 34.0 \mathrm{mmol})$. The resulting suspension was refluxed for $2 \mathrm{~h}$ and was then cooled to room temperature. To this mixture was successively and slowly added $\mathrm{H}_{2} \mathrm{O}(1.4 \mathrm{ml}), 15 \%$ aqueous sodium hydroxide (1.4 $\mathrm{ml})$, and $\mathrm{H}_{2} \mathrm{O}(4.2 \mathrm{~mL})$ to afford a white slurry which upon stirring separated the inorganic component into a solid mass. The organic layer can be easily separated by a simple filtration. The solid mass was triturated with dichloromethane $(3 \times 100 \mathrm{~mL})$ and filtered. The combined organic layers were dried over anhydrous magnesium sulfate and filtered. Evaporation of the solvent under reduced pressure afforded a colorless solid which was sufficiently pure and was used in the next step without further purification. Yield: $8.0 \mathrm{~g}(\sim 99 \%) ; \mathrm{mp} 78-80{ }^{\circ} \mathrm{C} ;{ }^{1} \mathrm{H}$ NMR $\left(\mathrm{CDCl}_{3}\right) \delta: 1.53(\mathrm{~s}, 3 \mathrm{H}), 3.09(\mathrm{~s}, 2 \mathrm{H}), 4.42(\mathrm{~s}, 2 \mathrm{H}), 6.66(\mathrm{~d}, 2 \mathrm{H}), 6.91(\mathrm{~d}, 2 \mathrm{H}), 7.23(\mathrm{~m}, 3 \mathrm{H}), 7.28$ 
(m, 3H), 7.52 (m, 2H); ${ }^{13} \mathrm{C}$ NMR $\left(\mathrm{CDCl}_{3}\right) \delta: 25.69,46.53,51.63,65.21,119.95,123.66,125.99$, $126.85,127.09,130.44,137.10,138.51,139.95,151.06$.

\section{Synthesis of $\mathrm{FX}_{2} \mathrm{~A}_{2}$.}

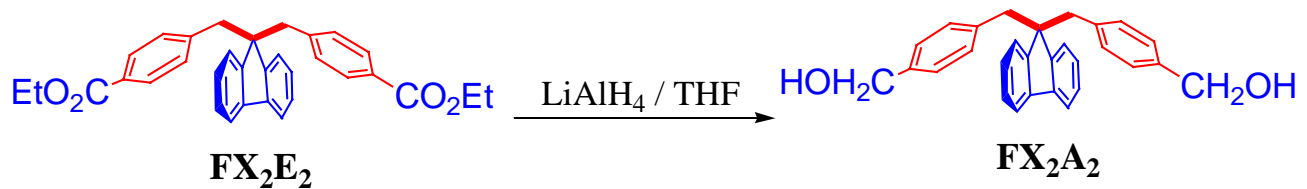

The di-ester $\mathbf{F X}_{2} \mathbf{E}_{2}(3.5 \mathrm{~g}, 7.0 \mathrm{mmol})$ in tetrahydrofuran $(75 \mathrm{~mL})$ was reacted with lithium aluminum hydride $(1.0 \mathrm{~g}, 24.0 \mathrm{mmol})$ as above to afford a colorless solid which was sufficiently pure and was used in the next step without any further purification. Yield: $2.5 \mathrm{~g}$ (86\%); mp 98-100 ${ }^{\circ} \mathrm{C} ;{ }^{1} \mathrm{H}$ NMR $\left(\mathrm{CDCl}_{3}\right) \delta: 3.38$ (s, 4H), $4.36(\mathrm{~s}, 4 \mathrm{H}), 6.56(\mathrm{~d}, 4 \mathrm{H}), 6.81(\mathrm{~d}, 4 \mathrm{H})$, $7.10(\mathrm{t}, 2 \mathrm{H}), 7.20(\mathrm{~m}, 4 \mathrm{H}), 7.36(\mathrm{~d}, 2 \mathrm{H}) ;{ }^{13} \mathrm{C} \mathrm{NMR}\left(\mathrm{CDCl}_{3}\right)$ 8: 45.53, 57.04, 65.38, 119.99, $124.73,126.09,126.49,127.23,130.57,136.67,138.45,141.03,148.11$.

\section{Synthesis of $\mathrm{F}_{2} \mathrm{X}_{3} \mathrm{~A}_{2}$.}

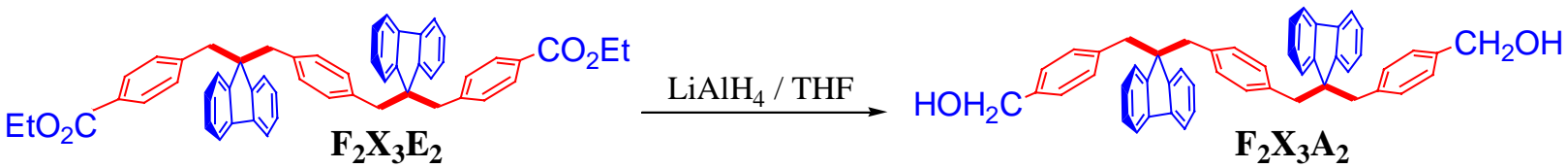

The di-ester $\mathbf{F}_{2} \mathbf{X}_{\mathbf{3}} \mathbf{E}_{\mathbf{2}}(3.6 \mathrm{~g}, 4.7 \mathrm{mmol})$ in tetrahydrofuran $(75 \mathrm{~mL})$ was reacted with lithium aluminum hydride $(0.76 \mathrm{~g}, 20 \mathrm{mmol})$ as above to afford $\mathbf{F}_{2} \mathbf{X}_{\mathbf{3}} \mathbf{A}_{\mathbf{2}}$ as a colorless solid which was sufficiently pure and was used in the next step without any further purification. Yield: $2.4 \mathrm{~g}(76 \%) ; \mathrm{mp} 126-128{ }^{\circ} \mathrm{C} ;{ }^{1} \mathrm{H}$ NMR $\left(\mathrm{CDCl}_{3}\right) \delta: 3.10$ (s, 4H), 3.22 (s, 4H), 4.37 (s, 4H), 6.29 (s, 4H), $6.57(\mathrm{~d}, 4 \mathrm{H}), 6.80(\mathrm{~d}, 4 \mathrm{H}), 7.18(\mathrm{~m}, 12 \mathrm{H}), 7.34(\mathrm{~m}, 4 \mathrm{H}) ;{ }^{13} \mathrm{C} \mathrm{NMR}\left(\mathrm{CDCl}_{3}\right) \delta: \quad 44.34$ $45.84,56.75,65.28,119.63,124.76,125.93,126.28,126.99,129.18,130.34,134.64,136.76$ $138.19,140.82,148.24$. 


\section{Synthesis of FXCM.}

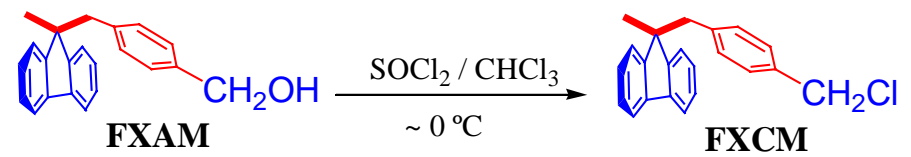

To a cooled $\left(\sim 0^{\circ} \mathrm{C}\right)$ solution of alcohol FXAM $(6.4 \mathrm{~g}, 21 \mathrm{mmol})$ in chloroform $(75 \mathrm{~mL})$ under an argon atmosphere was slowly added a solution of thionyl chloride $(1.8 \mathrm{ml}, 25 \mathrm{mmol})$ in chloroform $(50 \mathrm{ml})$ with the aid of a dropping funnel. The resulting mixture was stirred for 30 min. at $0{ }^{\circ} \mathrm{C}$ and for an additional $10 \mathrm{~h}$ at room temperature. To this mixture was then added slowly a $5 \%$ aqueous $\mathrm{NaHCO}_{3}$ solution $(150 \mathrm{~mL})$ and the resulting mixture was extracted with dichloromethane $(3 \times 100 \mathrm{~mL})$. The combined dichloromethane extracts were dried over anhydrous magnesium sulfate, filtered, and evaporated under reduced pressure to afford a white solid. The crude product was recrystallized from $\mathrm{CH}_{2} \mathrm{Cl}_{2} / \mathrm{CH}_{3} \mathrm{OH}$ to afford $\mathbf{F X C M}$ as a white crystalline solid. Yield: $6.7 \mathrm{~g}(98 \%) ; \mathrm{mp} 90-92{ }^{\circ} \mathrm{C} ;{ }^{1} \mathrm{H}$ NMR $\left(\mathrm{CDCl}_{3}\right) \delta: 1.56$ (s, 3H), 3.13 (s, 2H), $4.43(\mathrm{~s}, 2 \mathrm{H}), 6.69(\mathrm{~d}, 2 \mathrm{H}), 7.01(\mathrm{~d}, 2 \mathrm{H}), 7.29(\mathrm{~m}, 6 \mathrm{H}), 7.59(\mathrm{~m}, 2 \mathrm{H}) ;{ }^{13} \mathrm{C} \mathrm{NMR}\left(\mathrm{CDCl}_{3}\right) \delta$ : $25.81,46.61,46.72,51.76,120.17,123.85,127.08,127.35,127.73,130.78,135.24,138.22$ $140.12,151.09$.

\section{Synthesis of $\mathrm{FX}_{2} \mathrm{C}_{2}$.}

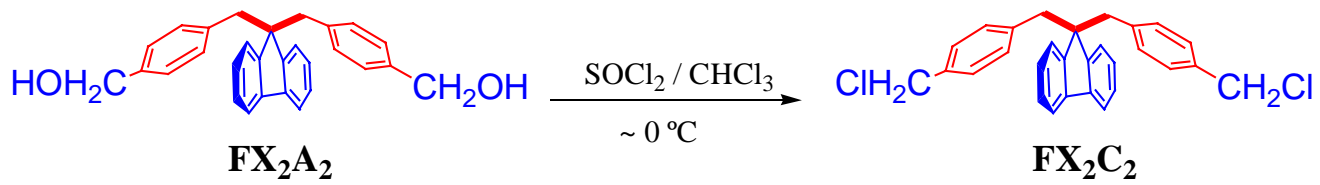

The di-alcohol $\mathbf{F X}_{2} \mathbf{A}_{2}$ (2.5 g, $\left.6 \mathrm{mmol}\right)$ in chloroform $(60 \mathrm{~mL})$ was reacted with a solution of thionyl chloride $(1.3 \mathrm{ml}, 18 \mathrm{mmol})$ in chloroform $(50 \mathrm{ml})$ at $0{ }^{\circ} \mathrm{C}$ as above to afford $\mathbf{F X}_{2} \mathbf{C}_{2}$ as a white solid which was recrystallized from $\mathrm{CH}_{2} \mathrm{Cl}_{2} / \mathrm{CH}_{3} \mathrm{OH}$ to afford a white crystalline solid. Yield: 2.2 g (77 \%); mp 183-185 ${ }^{\circ} \mathrm{C} ;{ }^{1} \mathrm{H}$ NMR $\left(\mathrm{CDCl}_{3}\right) \delta: 3.26$ (s, 4H), 4.26 (s, 4H), $6.54(\mathrm{~d}$, 
4H), $6.82(\mathrm{~d}, 4 \mathrm{H}), 7.16(\mathrm{~m}, 4 \mathrm{H}), 7.31(\mathrm{~m}, 4 \mathrm{H}),{ }^{13} \mathrm{C} \mathrm{NMR}\left(\mathrm{CDCl}_{3}\right) \delta: 45.38,46.37,56.76,119.93$, 124.52, 126.47, 127.23, 127.48, 130.55, 134.96, 137.35, 140.90, 147.78.

\section{Synthesis of $\mathrm{F}_{2} \mathbf{X}_{3} \mathbf{C}_{2}$.}

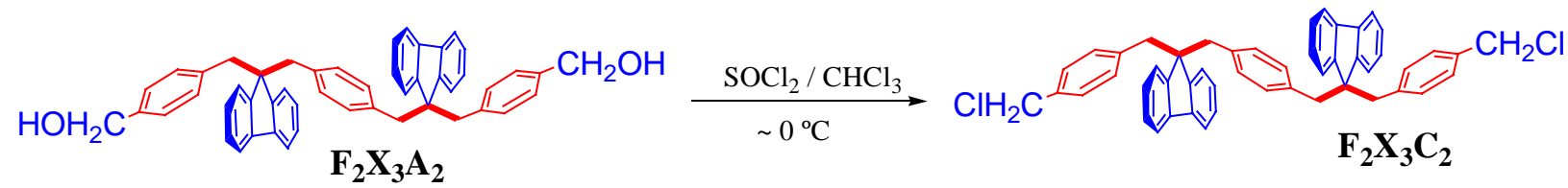

The di-alcohol $\mathbf{F}_{2} \mathbf{X}_{\mathbf{3}} \mathbf{A}_{\mathbf{2}}(2.4 \mathrm{~g}, 3.5 \mathrm{mmol})$ in chloroform $(50 \mathrm{~mL})$ was reacted with a solution of thionyl chloride $(0.8 \mathrm{ml}, 11 \mathrm{mmol})$ in chloroform $(50 \mathrm{ml})$ as above to afford $\mathbf{F}_{2} \mathbf{X}_{3} \mathbf{C}_{2}$ as a white solid. The crude material was purified by flash chromatography on silica gel using $15 \%$ ethyl acetate/hexanes as an eluent to afford a white solid. Yield: $1.4 \mathrm{~g}(56 \%)$; mp 176-178 ${ }^{\circ} \mathrm{C} ;{ }^{1} \mathrm{H}$ NMR $\left(\mathrm{CDCl}_{3}\right)$ 8: $3.04(\mathrm{~s}, 4 \mathrm{H}), 3.14(\mathrm{~s}, 4 \mathrm{H}) 4.28(\mathrm{~s}, 4 \mathrm{H}), 6.21(\mathrm{~s}, 4 \mathrm{H}), 6.49(\mathrm{~d}, 4 \mathrm{H}), 6.81$ (d, 4H), $7.13(\mathrm{~m}, 12 \mathrm{H}), 7.31(\mathrm{~m}, 4 \mathrm{H}) ;{ }^{13} \mathrm{C} \mathrm{NMR}\left(\mathrm{CDCl}_{3}\right) \delta: 44.37,45.78,46.41,56.69,119.65$, $124.71,126.31,127.04,127.42,129.18,130.45,134.59,134.79,137.62,140.80,148.10$.

\section{Synthesis of $\mathrm{F}_{2} \mathrm{XHM}$.}
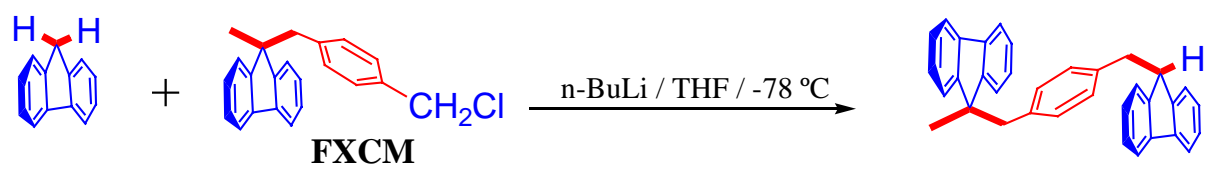

$\mathrm{F}_{2} \mathrm{XHM}$

To a solution of fluorene $(2.1 \mathrm{~g}, 12.5 \mathrm{mmol})$ in anhydrous tetrahydrofuran $(20 \mathrm{~mL})$ cooled to $-78{ }^{\circ} \mathrm{C}$, under an argon atmosphere, was added dropwise $n$-butyllithium (2.5 $\mathrm{M}$ in hexanes, $5.1 \mathrm{ml})$. After $10 \mathrm{~min}$. of stirring, solid FXCM (1.9 g, $6.0 \mathrm{mmol})$ was added and the resulting mixture was stirred for $15 \mathrm{~min}$ at $-78{ }^{\circ} \mathrm{C}$. The cooling bath was then removed and the reaction mixture was stirred for an additional $1 \mathrm{~h}$. It was then poured onto water $(100 \mathrm{~mL})$ and the resulting mixture was extracted with dichloromethane $(3 \times 100 \mathrm{~mL})$. The combined 
dichloromethane extracts were dried over anhydrous magnesium sulfate, filtered, and evaporated under reduced pressure to afford a white solid material. The crude material was purified by flash chromatography on silica gel using 5\% ethyl acetate/hexanes as an eluent to afford a white solid. Yield: $5.2 \mathrm{~g} \mathrm{(92 \% );} \mathrm{mp} \mathrm{122-124}{ }^{\circ} \mathrm{C} ;{ }^{1} \mathrm{H}$ NMR $\left(\mathrm{CDCl}_{3}\right) \delta: 1.61$ (s, 3H), 2.89 (d, 2H), 3.18 (s, 2H), $4.05(\mathrm{t}, 1 \mathrm{H}) 6.58(\mathrm{~d}, 2 \mathrm{H}), 6.82(\mathrm{~d}, 2 \mathrm{H}), 6.90(\mathrm{~d}, 2 \mathrm{H}), 7.19(\mathrm{t}, 2 \mathrm{H}) 7.33(\mathrm{~m}, 8 \mathrm{H}), 7.63(\mathrm{~m}$, 2H), $7.71(\mathrm{~d}, 2 \mathrm{H}) ;{ }^{13} \mathrm{C}$ NMR $\left(\mathrm{CDCl}_{3}\right) \delta: 25.90,40.23,46.99,49.21,52.09,120.09,124.02$, $125.20,126.91,127.14,127.36,128.59,130.30,135.88,137.63,140.41,141.06,147.18,151.40$.

\section{Synthesis of $F_{2} X_{2} E M$.}
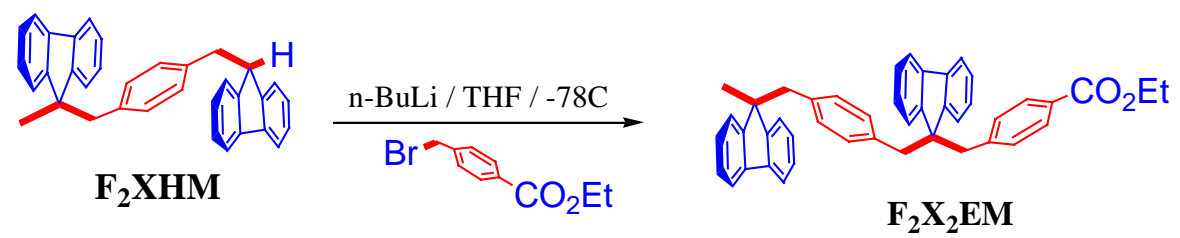

To a cooled $\left(-78{ }^{\circ} \mathrm{C}\right)$ solution of $\mathbf{F}_{2} \mathbf{X H M}(4.0 \mathrm{~g}, 8.9 \mathrm{mmol})$ in anhydrous tetrahydrofuran (30 $\mathrm{mL})$, under an argon atmosphere, was added $n$-butyllithium $(2.5 \mathrm{M}$ in hexanes, $3.6 \mathrm{~mL})$ dropwise. After 10 min., solid ethyl-4-(bromomethyl)benzoate (2.2 g, 8.9 mmol) was added and resulting mixture was stirred for $15 \mathrm{~min}$ at $-78{ }^{\circ} \mathrm{C}$. The cooling bath was removed and the reaction mixture was stirred for an additional $1 \mathrm{~h}$. It was then poured onto water $(100 \mathrm{~mL})$ and the aqueous layer was extracted with dichloromethane $(3 \mathrm{x} 100 \mathrm{~mL})$. The combined dichloromethane extracts were dried over anhydrous magnesium sulfate, filtered, and evaporated under reduced pressure to afford a yellow solid material. The crude material was purified by flash chromatography on silica gel using $10 \%$ ethyl acetate/hexanes as an eluent to afford $\mathbf{F}_{2} \mathbf{X}_{2} \mathbf{E M}$ as a white solid. Yield: $5.4 \mathrm{~g}(98 \%) ; \mathrm{mp} 188-190{ }^{\circ} \mathrm{C} ;{ }^{1} \mathrm{H} \mathrm{NMR}\left(\mathrm{CDCl}_{3}\right) \delta: 1.21(\mathrm{t}, 3 \mathrm{H})$, $1.32(\mathrm{~s}, 3 \mathrm{H}), 2.82(\mathrm{~s}, 2 \mathrm{H}), 3.08(\mathrm{~s}, 2 \mathrm{H}) 3.24(\mathrm{~s}, 2 \mathrm{H}), 4.15(\mathrm{q}, 2 \mathrm{H}), 6.28(\mathrm{q}, 4 \mathrm{H}), 6.40(\mathrm{~d}, 2 \mathrm{H}) 7.05$ $(\mathrm{d}, 2 \mathrm{H}), 7.16(\mathrm{~m}, 10 \mathrm{H}), 7.32(\mathrm{~m}, 2 \mathrm{H}), 7.45(\mathrm{~d}, 2 \mathrm{H}), 7.51(\mathrm{~d}, 2 \mathrm{H}) ;{ }^{13} \mathrm{C} \mathrm{NMR}\left(\mathrm{CDCl}_{3}\right) \delta: 24.69$ 
$44.38,44.40,44.68,45.81,46.53,51.36,56.78,119.59,119.69,123.76,124.21,124.68,125.84$, $126.22,126.67,126.73,126.91,126.95,127.44,128.71,129.07,129.13,129.47,130.23,134.59$, $135.14,136.53,138.29,139.73,140.80,148.18,148.30,151.20,166.23$.

\section{Synthesis of $\mathbf{F}_{2} \mathbf{X}_{2} \mathrm{AM}$.}

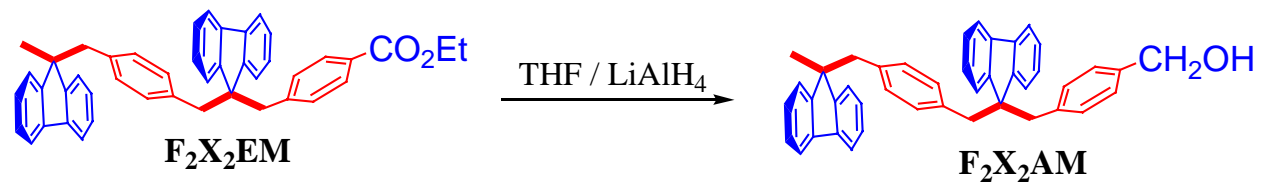

A solution of ester $\mathbf{F}_{2} \mathbf{X}_{2} \mathbf{E M}$ (7.9 g, $13.0 \mathrm{mmol}$ ), obtained above, in tetrahydrofuran (75 $\mathrm{mL}$ ) was reacted with lithium aluminum hydride $(0.76 \mathrm{~g}, 20 \mathrm{mmol})$ as above to afford $\mathbf{F}_{2} \mathbf{X}_{2} \mathbf{A M}$ as a colorless solid, which was sufficiently pure and was used in the next step without further purification. Yield: $6.55 \mathrm{~g}(88 \%)$; mp 130-132 ${ }^{\circ} \mathrm{C} ;{ }^{1} \mathrm{H} \mathrm{NMR}\left(\mathrm{CDCl}_{3}\right) \delta: 1.37$ (s, 3H), $2.86(\mathrm{~s}$, 2H), $3.15(\mathrm{~s}, 2 \mathrm{H}), 3.25(\mathrm{~s}, 2 \mathrm{H}), 4.31(\mathrm{~d}, 2 \mathrm{H}), 6.33(\mathrm{~s}, 4 \mathrm{H}), 6.59(\mathrm{~d}, 2 \mathrm{H}), 6.82(\mathrm{~d}, 2 \mathrm{H}), 6.95(\mathrm{~d}$, 2H), $7.23(\mathrm{~m}, 10 \mathrm{H}), 7.40(\mathrm{~d}, 2 \mathrm{H}), 7.64(\mathrm{~d}, 2 \mathrm{H}) ;{ }^{13} \mathrm{C} \mathrm{NMR}\left(\mathrm{CDCl}_{3}\right)$ 8: 24.69, 44.38, 45.81, 46.53, $51.36,56.78,68.05,119.59,119.69,123.76,124.21,124.68,125.84,126.22,126.67,126.73$ $126.91,126.95,127.44,128.71,129.07,129.13,129.47,130.23,134.59,135.14,136.53,138.29$, $139.73,140.80,148.18,148.30,151.20$.

\section{Synthesis of $\mathrm{F}_{2} \mathrm{X}_{2} \mathrm{CM}$.}

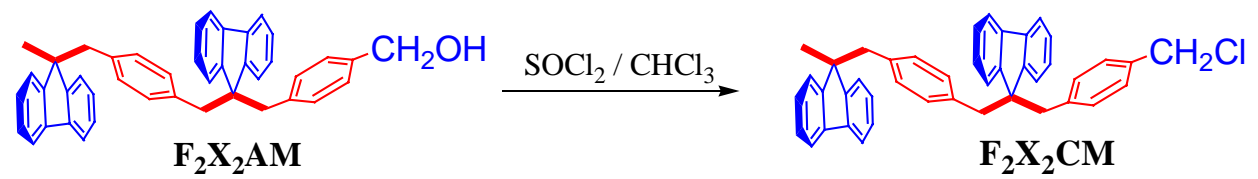

A solution of alcohol $\mathbf{F}_{2} \mathbf{X}_{2} \mathbf{A M}(6.6 \mathrm{~g}, 11.5 \mathrm{mmol})$, obtained above, in chloroform (75 $\mathrm{mL})$ was reacted with a solution of thionyl chloride $(1.5 \mathrm{ml}, 20 \mathrm{mmol})$ in chloroform $(50 \mathrm{ml})$ as above to afford $\mathbf{F}_{2} \mathbf{X}_{2} \mathbf{C M}$ as a white solid. The crude material was purified by flash chromatography on silica gel using $10 \%$ ethyl acetate/hexanes as an eluent to afford a colorless 
crystalline solid. Yield: $6.4 \mathrm{~g}(95 \%) ; \mathrm{mp} 100-102{ }^{\circ} \mathrm{C} ;{ }^{1} \mathrm{H} \mathrm{NMR}\left(\mathrm{CDCl}_{3}\right) \delta: 1.37$ (s, 3H), 2.86 (s, 2H) $3.14(\mathrm{~s}, 2 \mathrm{H}), 3.24(\mathrm{~s}, 2 \mathrm{H}), 4.30(\mathrm{~s}, 2 \mathrm{H}), 6.31(\mathrm{~s}, 4 \mathrm{H}), 6.57(\mathrm{~d}, 2 \mathrm{H}), 6.85(\mathrm{~d}, 2 \mathrm{H}), 7.03(\mathrm{~d}, 2 \mathrm{H})$, $7.21(\mathrm{~m}, 10 \mathrm{H}), 7.35(\mathrm{~d}, 2 \mathrm{H}), 7.55(\mathrm{~d}, 2 \mathrm{H}) ;{ }^{13} \mathrm{C} \mathrm{NMR}\left(\mathrm{CDCl}_{3}\right) \delta: \quad 24.77,44.48,45.79,46.35$, $46.58,51.43,56.74,119.66,119.72,123.80,124.68,126.30,126.72,126.96,127.05,127.42$, 129.13, 129.17, 130.44, 134.56, 134.81, 135.24, 137.63, 139.80, 140.86, 148.11, 151.23.

\section{Synthesis of $\mathbf{F}_{3} \mathbf{X}_{2} \mathbf{H M}$.}

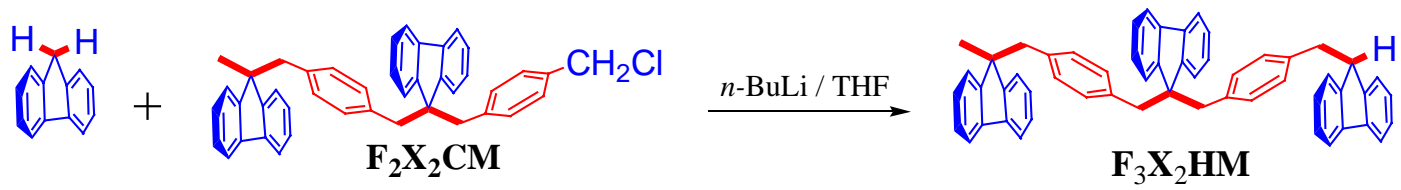

To a cooled $\left(-78{ }^{\circ} \mathrm{C}\right)$ solution of fluorene $(1.6 \mathrm{~g}, 9.5 \mathrm{mmol})$ in anhydrous tetrahydrofuran (30 $\mathrm{mL}$ ), under an argon atmosphere, was added dropwise a solution of $n$-butyllithium in hexanes $(2.5 \mathrm{M}, 3.8 \mathrm{~mL})$ to afford an orange-colored solution. After $10 \mathrm{~min}$. of stirring, solid $\mathbf{F}_{2} \mathbf{X}_{2} \mathbf{C M}$ (5.6 g, $9.5 \mathrm{mmol}$ ) was added and the resulting mixture was stirred for $15 \mathrm{~min}$. The cooling bath was removed and the reaction mixture was stirred for an additional $1 \mathrm{~h}$. It was then poured onto water $(100 \mathrm{~mL})$ and the aqueous layer was extracted with dichloromethane $(3 \times 100$ $\mathrm{mL}$ ). The combined dichloromethane extracts were dried over anhydrous magnesium sulfate, filtered, and evaporated under reduced pressure to afford a white solid material which was sufficiently pure and was used in the next step without further purification. Yield: $6.7 \mathrm{~g} \mathrm{(98 \% );}$ mp 136-138 ${ }^{\circ} \mathrm{C} ;{ }^{1} \mathrm{H}$ NMR $\left(\mathrm{CDCl}_{3}\right) \delta: 1.39$ (s, 3H), 2.80 (d, 2H), 2.88 (s, 2H), 3.19 (s, 2H) 3.28 (s, 2H), $3.97(\mathrm{t}, 1 \mathrm{H}), 6.75(\mathrm{q}, 4 \mathrm{H}), 6.49(\mathrm{~d}, 2 \mathrm{H}) 6.75(\mathrm{q}, 4 \mathrm{H}), 7.06(\mathrm{~d}, 2 \mathrm{H}), 7.21(\mathrm{~m}, 12 \mathrm{H}), 7.43$ (m, 4H), $7.59(\mathrm{~d}, 2 \mathrm{H}), 7.70(\mathrm{~d}, 2 \mathrm{H}) ;{ }^{13} \mathrm{C} \mathrm{NMR}\left(\mathrm{CDCl}_{3}\right) \delta: 25.88,31.86,39.95,45.41,45.81$, $48.90,57.10,68.19,119.72,119.74,124.76,124.87,125.17,125.50,126.38,126.50,126.59$, 
127.03, 127.14, 127.41, 127.60, 127.63, 128.19, 129.05, 129.07, 130.01, 131.89, 132.88, 135.01, $137.28,140.69,141.03,146.84,148.23$.

\section{Synthesis of $F_{3} X_{3} E M$.}

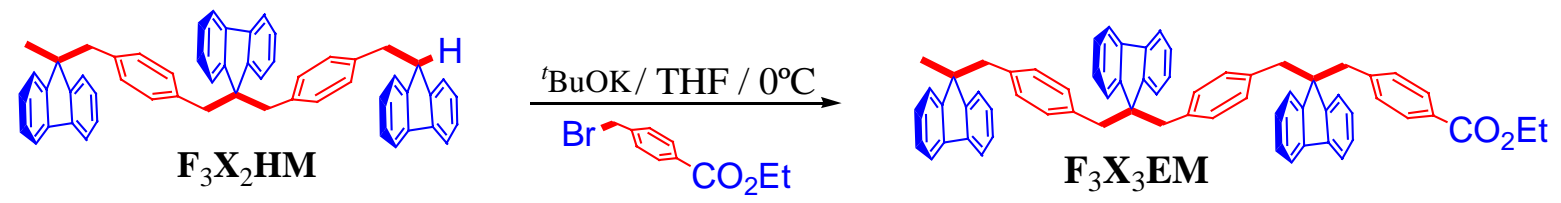

To a cooled solution $\left(\sim 0{ }^{\circ} \mathrm{C}\right)$ of $\mathbf{F}_{3} \mathbf{X}_{2} \mathbf{H M}(5.0 \mathrm{~g}, 7 \mathrm{mmol})$ in anhydrous tetrahydrofuran $(150 \mathrm{ml})$ solid potassium tert-butoxide $(2.6 \mathrm{~g}, 2.3 \mathrm{mmol})$ was added. After $10 \mathrm{~min}$. of stirring, solid ethyl-4-(bromomethyl)benzoate $(1.7 \mathrm{~g}, 7 \mathrm{mmol})$ was added and the resulting mixture was stirred for an additional $15 \mathrm{~min}$. at $0{ }^{\circ} \mathrm{C}$. The cooling bath was removed and the reaction mixture was stirred for $12 \mathrm{~h}$. It was then poured onto water $(100 \mathrm{~mL})$ and the aqueous layer was extracted with dichloromethane $(3 \times 100 \mathrm{~mL})$. The combined dichloromethane extracts were dried over anhydrous magnesium sulfate, filtered, and evaporated under reduced pressure to afford a yellow solid material. The crude material was purified by flash chromatography on silica gel using 5\% ethyl acetate/hexanes as an eluent to afford $\mathbf{F}_{\mathbf{3}} \mathbf{X}_{\mathbf{3}} \mathbf{E M}$ as a white solid. Yield: $6.0 \mathrm{~g}(98 \%) ; \mathrm{mp} 196-198{ }^{\circ} \mathrm{C} ;{ }^{1} \mathrm{H}$ NMR $\left(\mathrm{CDCl}_{3}\right) \delta: 1.22(\mathrm{t}, 3 \mathrm{H}), 1.34(\mathrm{~s}, 3 \mathrm{H}), 2.84(\mathrm{~s}, 2 \mathrm{H}), 3.07$ (t, 6H) $3.19(\mathrm{~s}, 2 \mathrm{H}), 4.18(\mathrm{q}, 2 \mathrm{H}), 6.27(\mathrm{~d}, 8 \mathrm{H}), 6.89(\mathrm{~d}, 2 \mathrm{H}) 7.09(\mathrm{~m}, 18 \mathrm{H}), 7.39(\mathrm{~m}, 4 \mathrm{H}), 7.59$ $(\mathrm{d}, 2 \mathrm{H}) ;{ }^{13} \mathrm{C} \mathrm{NMR}\left(\mathrm{CDCl}_{3}\right) \delta: 24.33,30.56,42.54,44.23,44.78,44.89,45.77,51.35,56.72$, $56.79,119.36,119.66,119.89,123.44,124.63,124.69,125.33,126.13,126.21,126.66,126.86$ $126.99,129.02,129.18,130.28,134.59,134.88,135.28,136.68,138.14,139.75,140.76,140.83$ $148.49,148.61,151.39,166.59$. 


\section{Synthesis of $\mathbf{F}_{3} \mathbf{X}_{3} \mathbf{A M}$.}

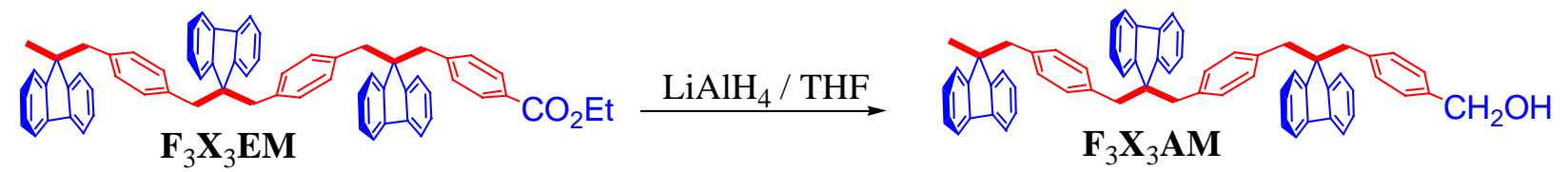

A solution of ester $\mathbf{F}_{\mathbf{3}} \mathbf{X}_{\mathbf{3}} \mathbf{E M}(12.0 \mathrm{~g}, 16.3 \mathrm{mmol})$, obtained above, in tetrahydrofuran (100 $\mathrm{mL})$ was reacted with lithium aluminum hydride $(1.9 \mathrm{~g}, 50 \mathrm{mmol})$ as above to afford $\mathbf{F}_{\mathbf{3}} \mathbf{X}_{\mathbf{3}} \mathbf{A M}$ as a colorless solid, which was used in the next step without further purification. Yield: $10.5 \mathrm{~g}$ (93\%); mp 144-146 ${ }^{\circ} \mathrm{C} ;{ }^{1} \mathrm{H}$ NMR $\left(\mathrm{CDCl}_{3}\right) \delta: 1.36(\mathrm{~s}, 3 \mathrm{H}), 2.82(\mathrm{~s}, 2 \mathrm{H}), 3.05(\mathrm{t}, 6 \mathrm{H}), 3.19(\mathrm{~s}, 2 \mathrm{H})$, $4.35(\mathrm{~d}, 2 \mathrm{H}), 6.29(\mathrm{~d}, 8 \mathrm{H}), 6.51(\mathrm{~d}, 2 \mathrm{H}), 6.82(\mathrm{~d}, 2 \mathrm{H}), 7.18(\mathrm{~m}, 18 \mathrm{H}), 7.39(\mathrm{~m}, 4 \mathrm{H}), 7.55(\mathrm{~d}, 2 \mathrm{H})$; ${ }^{13} \mathrm{C} \mathrm{NMR}\left(\mathrm{CDCl}_{3}\right) \delta: 24.63,30.55,44.78,44.89,45.86,46.55,51.35,56.69,65.12,119.36$, $119.61,119.73,123.80,124.72,124.87,125.85,126.14,126.21,126.68,126.85,126.92,129.02$, $129.15,130.26,134.49,134.82,135.05,136.65,138.19,139.73,140.74,140.78,148.20,148.43$, 151.24 .

\section{Synthesis of $\mathrm{F}_{3} \mathbf{X}_{3} \mathbf{C M}$.}

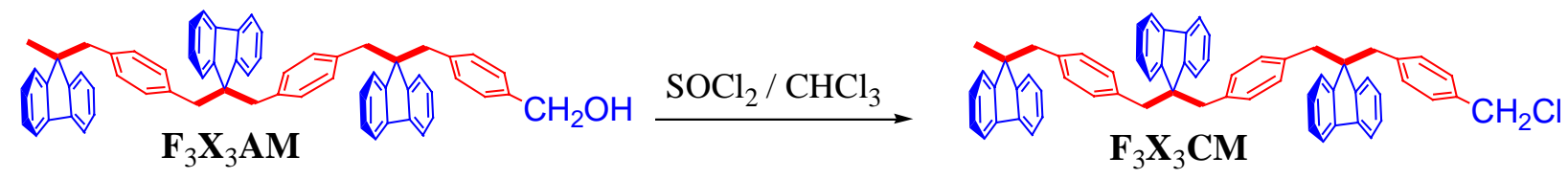

A solution of alcohol $\mathbf{F}_{3} \mathbf{X}_{\mathbf{3}} \mathbf{A M}(5.5 \mathrm{~g}, 6.6 \mathrm{mmol})$, obtained above, in chloroform $(75 \mathrm{~mL})$ was reacted with a solution of thionyl chloride $(1.5 \mathrm{ml}, 20 \mathrm{mmol})$ in chloroform $(50 \mathrm{ml})$ as above to afford $\mathbf{F}_{\mathbf{3}} \mathbf{X}_{\mathbf{3}} \mathbf{C M}$ as a white solid. The crude material was purified by flash chromatography on silica gel using $12 \%$ ethyl acetate/hexanes as an eluent to afford a colorless solid. Yield: $5.5 \mathrm{~g}$ (97\%); mp 110-112 ${ }^{\circ} \mathrm{C} ;{ }^{1} \mathrm{H} \mathrm{NMR}\left(\mathrm{CDCl}_{3}\right) \delta: 1.37(\mathrm{~s}, 3 \mathrm{H}), 2.86(\mathrm{~s}, 2 \mathrm{H}) 3.14(\mathrm{t}, 6 \mathrm{H}), 3.24(\mathrm{~s}, 2 \mathrm{H})$, $4.34(\mathrm{~s}, 2 \mathrm{H}), 6.29(\mathrm{~d}, 8 \mathrm{H}), 6.51(\mathrm{~d}, 2 \mathrm{H}), 6.82(\mathrm{~d}, 2 \mathrm{H}), 7.18(\mathrm{~m}, 18 \mathrm{H}), 7.39(\mathrm{~m}, 4 \mathrm{H}), 7.55(\mathrm{~d}, 2 \mathrm{H}))$;

${ }^{13} \mathrm{C} \mathrm{NMR}\left(\mathrm{CDCl}_{3}\right) \delta:$ 24.77, 30.67, 44.48, 44.72, 45.79, 46.35, 46.58, 51.43, 56.74, 119.33, 
$119.68,119.76,123.86,124.74,124.89,125.88,126.14,126.24,126.65,126.81,126.93,129.10$, $129.16,130.28,134.48,134.83,135.28,136.05,138.19,139.74,140.05,140.70,148.23,148.45$, 151.39.

\section{Synthesis of $\mathbf{F}_{4} \mathbf{X}_{3} \mathbf{H M}$.}

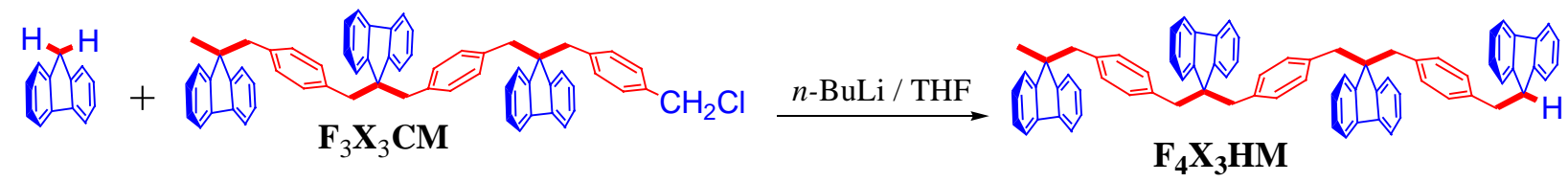

To a solution of fluorene $(0.5 \mathrm{~g}, 3.0 \mathrm{mmol})$ in anhydrous tetrahydrofuran $(30 \mathrm{~mL})$ at -78 ${ }^{\circ} \mathrm{C}$ was reacted with $n$-butyllithium $(2.5 \mathrm{M}$ in hexanes, $1.2 \mathrm{~mL})$ and $\mathbf{F}_{\mathbf{3}} \mathbf{X}_{\mathbf{3}} \mathbf{C M}(2.6 \mathrm{~g}, 3.0 \mathrm{mmol})$ as above to afford an yellow solid material. The crude material was purified by flash chromatography on silica gel using $15 \%$ ethyl acetate/hexanes as an eluent to afford $\mathbf{F}_{\mathbf{4}} \mathbf{X}_{\mathbf{3}} \mathbf{H M}$ as a white solid. Yield: $6.7 \mathrm{~g}(68 \%)$; mp 148-150 ${ }^{\circ} \mathrm{C} ;{ }^{1} \mathrm{H} \mathrm{NMR}\left(\mathrm{CDCl}_{3}\right) \delta: 1.35$ (s, 3H), $2.76(\mathrm{~d}$, 2H), $2.83(\mathrm{~s}, 2 \mathrm{H}), 3.06(\mathrm{~d}, 6 \mathrm{H}) 3.22(\mathrm{~s}, 2 \mathrm{H}), 3.94(\mathrm{t}, 1 \mathrm{H}), 6.31(\mathrm{~m}, 8 \mathrm{H}), 6.46(\mathrm{~d}, 2 \mathrm{H}) 6.73(\mathrm{q}, 4 \mathrm{H})$, $7.06(\mathrm{~d}, 2 \mathrm{H}), 7.21(\mathrm{~m}, 20 \mathrm{H}), 7.40(\mathrm{~m}, 4 \mathrm{H}), 7.57(\mathrm{~d}, 2 \mathrm{H}), 7.68(\mathrm{~d}, 2 \mathrm{H}) ;{ }^{13} \mathrm{C}$ NMR $\left(\mathrm{CDCl}_{3}\right) \delta$ : $24.65,39.98,44.47,44.83,44.95,45.62,46.58,48.96,51.38,56.73,56.87,119.41,119.46$, $123.83,124.90,126.18,126.25,126.61,126.70,126.89,126.95,127.03,128.15,129.13,129.24$ $129.94,134.69,134.88,135.08,135.23,137.20,139.77,140.70,140.83,140.93,146.85,148.40$, $148.48,151.28$.

\section{Synthesis of Z2.}

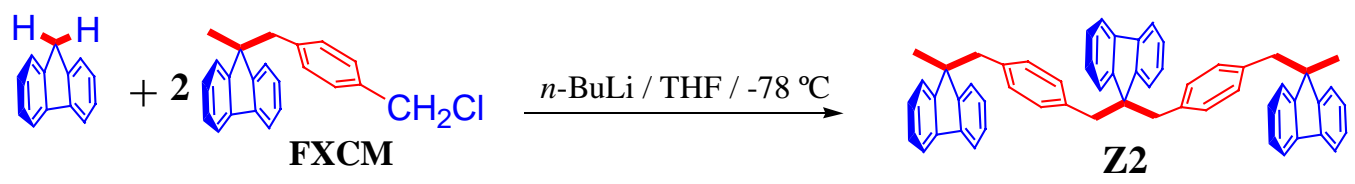

A solution of fluorene $(1.0 \mathrm{~g}, 6.0 \mathrm{mmol})$ in anhydrous tetrahydrofuran $(20 \mathrm{ml})$ was reacted with $n$-butyllithium $(2.5 \mathrm{M}$ in hexanes, $3.0 \mathrm{ml})$ at $-78{ }^{\circ} \mathrm{C}$. After $10 \mathrm{~min}$. of stirring, solid 
FXCM (1.9 g, $6.0 \mathrm{mmol}$ ) was added and the mixture was stirred for an additional $15 \mathrm{~min}$. at -78 ${ }^{\circ} \mathrm{C}$, then warmed to room temperature. The resulting reaction mixture was re-cooled to $-78{ }^{\circ} \mathrm{C}$ and $n$-butyllithium (2.5 M in hexanes, $3.0 \mathrm{ml}$ ) was added dropwise and the resulting dark-orange mixture was stirred for $10 \mathrm{~min}$. To this solution, solid FXCM $(1.9 \mathrm{~g}, 6.0 \mathrm{mmol})$ was added and the mixture was stirred for $15 \mathrm{~min}$. at $-78{ }^{\circ} \mathrm{C}$. The cooling bath was removed and the reaction mixture was stirred for an additional $1 \mathrm{~h}$. It was then poured onto water $(200 \mathrm{~mL})$ and the aqueous layer was extracted with dichloromethane $\left(\begin{array}{llll}3 & \mathrm{x} & 100 & \mathrm{~mL}\end{array}\right)$. The combined dichloromethane extracts were dried over anhydrous magnesium sulfate, filtered, and evaporated under reduced pressure to afford a white solid material. The crude material was purified by flash chromatography on silica gel using $2 \%$ ethyl acetate/hexanes as an eluent to afford $\mathbf{Z 2}$ as a white solid. Yield: $4.0 \mathrm{~g}(91 \%)$; mp 208-210 ${ }^{\circ} \mathrm{C} ;{ }^{1} \mathrm{H}$ NMR $\left(\mathrm{CDCl}_{3}\right) \delta$ : 1.29 (s, 6H), 2.77 (s, 4H), 3.01 $(\mathrm{s}, 4 \mathrm{H}), 6.24(\mathrm{~s}, 8 \mathrm{H}), 6.93(\mathrm{~d}, 4 \mathrm{H}), 7.03(\mathrm{~m}, 2 \mathrm{H}), 7.15(\mathrm{~m}, 12 \mathrm{H}) 7.33(\mathrm{~m}, 2 \mathrm{H}), 7.49(\mathrm{~d}, 4 \mathrm{H}),{ }^{13} \mathrm{C}$ NMR $\left(\mathrm{CDCl}_{3}\right) \delta: 24.91,44.98,45.24,46.84,51.63,56.91,119.66,120.02,124.01,125.42$, 126.40, 126.96, 127.11, 127.02, 129.32, 129.36, 134.92, 135.12, 135.02, 140.00, 140.03, 141.05, 148.71, 151.5.

\section{Synthesis of Z3.}

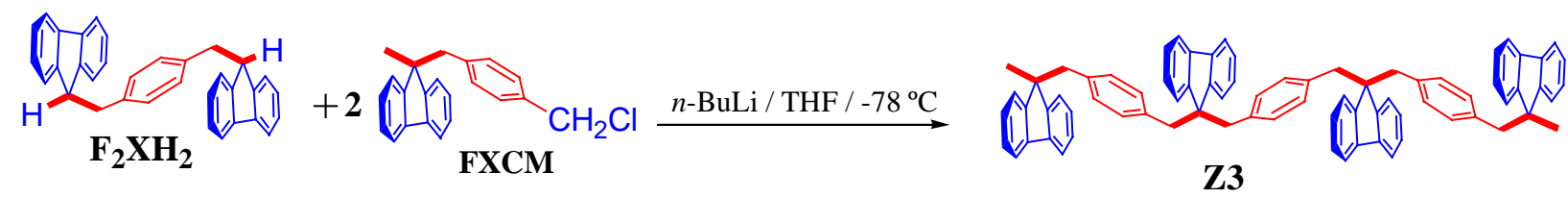

To a cooled $\left(-78{ }^{\circ} \mathrm{C}\right)$ solution of $\mathbf{F}_{2} \mathbf{X H}_{\mathbf{2}}(1.2 \mathrm{~g}, 2.8 \mathrm{mmol})$ in anhydrous tetrahydrofuran $(40 \mathrm{~mL})$ under an argon atmosphere was added a solution of $n$-butyllithium in hexanes $(2.5 \mathrm{M}$, $2.5 \mathrm{~mL})$. After $10 \mathrm{~min}$. of stirring, FXCM $(1.8 \mathrm{~g}, 5.6 \mathrm{mmol})$ was added and the resulting mixture was stirred for $15 \mathrm{~min}$. The cooling bath was removed and the reaction mixture was 
stirred for an additional $1 \mathrm{~h}$. It was then poured onto water $(200 \mathrm{~mL})$ and the aqueous layer was extracted with dichloromethane $(3 \times 100 \mathrm{~mL})$. The combined dichloromethane extracts were dried over anhydrous magnesium sulfate, filtered, and evaporated under reduced pressure to afford a white solid. The crude material was purified by flash chromatography on silica gel using $10 \%$ ethyl acetate/hexanes as an eluent to afford $\mathbf{Z 3}$ as a white solid which was recrystallized from a mixture of $\mathrm{CHCl}_{3} / \mathrm{CH}_{3} \mathrm{OH}$ to afford a white crystalline solid. Yield: $2.6 \mathrm{~g}$ (92\%); mp 195-197 ${ }^{\circ} \mathrm{C} ;{ }^{1} \mathrm{H}$ NMR $\left(\mathrm{CDCl}_{3}\right) \delta: 1.36(\mathrm{~s}, 6 \mathrm{H}), 2.83(\mathrm{~s}, 4 \mathrm{H}), 3.03(\mathrm{~d}, 8 \mathrm{H}), 6.28(\mathrm{~d}$, 12H), $7.06(\mathrm{~m}, 10 \mathrm{H}), 7.21(\mathrm{~m}, 14 \mathrm{H}), 7.38(\mathrm{~m}, 4 \mathrm{H}) 7.56(\mathrm{~d}, 4 \mathrm{H}) ;{ }^{13} \mathrm{C} \mathrm{NMR}\left(\mathrm{CDCl}_{3}\right) \delta: 24.90$ 44.98, 45.24, 46.84, 51.63, 56.92, 119.68, 120.02, 124.08, 125.14, 126.39, 126.96, 127.11, 127.20, 129.32, 129.36, 134.98, 135.12, 135.30, 140.04, 140.02, 141.04, 148.73, 151.53.

\section{Synthesis of Z4.}

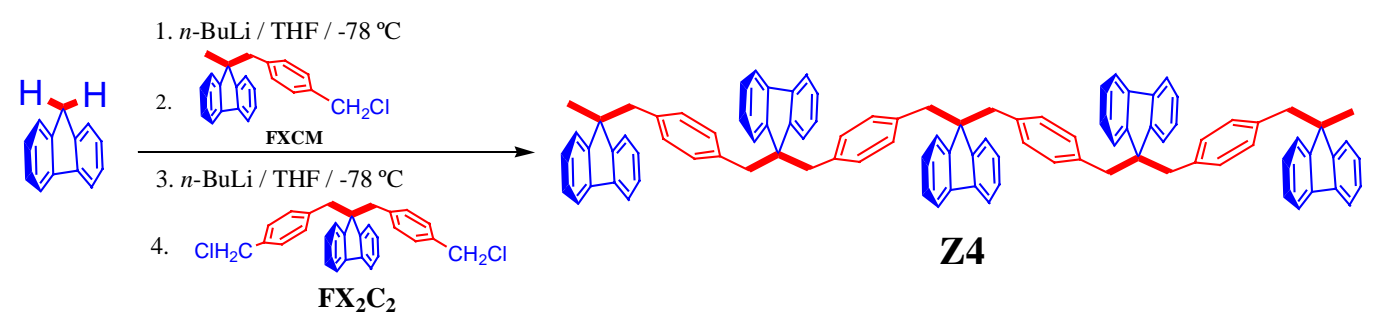

To a cold $\left(-78{ }^{\circ} \mathrm{C}\right)$ solution of fluorene $(1.6 \mathrm{~g}, 9.7 \mathrm{mmol})$ in anhydrous tetrahydrofuran (40 ml), $n$-butyllithium (2.5 $\mathrm{M}$ in hexanes, $4.0 \mathrm{ml}$ ) was added dropwise under an argon atmosphere. After $10 \mathrm{~min}$. of stirring, solid FXCM (3.1 g, $9.7 \mathrm{mmol})$ was added and the mixture was stirred for $15 \mathrm{~min}$. at $-78{ }^{\circ} \mathrm{C}$, then warmed to room temperature. The resulting reaction mixture was again cooled to $-78{ }^{\circ} \mathrm{C}$ and $n$-butyllithium (2.5 M in hexanes, $\left.4.0 \mathrm{ml}\right)$ was added dropwise. The resulting dark-orange mixture was stirred for $10 \mathrm{~min}$. and solid $\mathbf{F X}_{\mathbf{2}} \mathbf{C}_{2}(2.15 \mathrm{~g}$, $4.85 \mathrm{mmol}$ ) was added and the mixture was stirred for $15 \mathrm{~min}$ at $-78{ }^{\circ} \mathrm{C}$. The cooling bath was removed and the reaction mixture was stirred for an additional $1 \mathrm{~h}$. It was then poured onto 
water $(200 \mathrm{~mL})$ and the aqueous layer was extracted with dichloromethane $(3 \mathrm{x} 100 \mathrm{~mL})$. The combined dichloromethane extracts were dried over anhydrous magnesium sulfate, filtered, and evaporated under reduced pressure to afford a white solid. The crude material was purified by flash chromatography on silica gel using $12 \%$ ethyl acetate/hexanes as an eluent to afford $\mathbf{Z 4}$ as a white solid. Yield: $3.1 \mathrm{~g}(25 \%)$; mp 184-186 ${ }^{\circ} \mathrm{C} ;{ }^{1} \mathrm{H}$ NMR $\left(\mathrm{CDCl}_{3}\right) \delta: 1.38(\mathrm{~s}, 6 \mathrm{H}), 2.82(\mathrm{~s}, 4 \mathrm{H})$, $3.01(\mathrm{~m}, 12 \mathrm{H}), 6.23(\mathrm{~d}, 16 \mathrm{H}), 7.03(\mathrm{~m}, 10 \mathrm{H}), 7.20(\mathrm{~m}, 20 \mathrm{H}), 7.40(\mathrm{~m}, 6 \mathrm{H}), 7.57(\mathrm{~d}, 4 \mathrm{H}) ;{ }^{13} \mathrm{C}$ NMR $\left(\mathrm{CDCl}_{3}\right) \delta: 24.64,44.72,46.60,51.39,56.61,56.69,119.42,119.77,123.84,124.90$ $126.14,126.71,126.87,126.95,129.08,134.71,134.76,134.90,135.08,139.77,140.74,140.80$, $148.50,151.32$.

\section{Synthesis of Z5.}

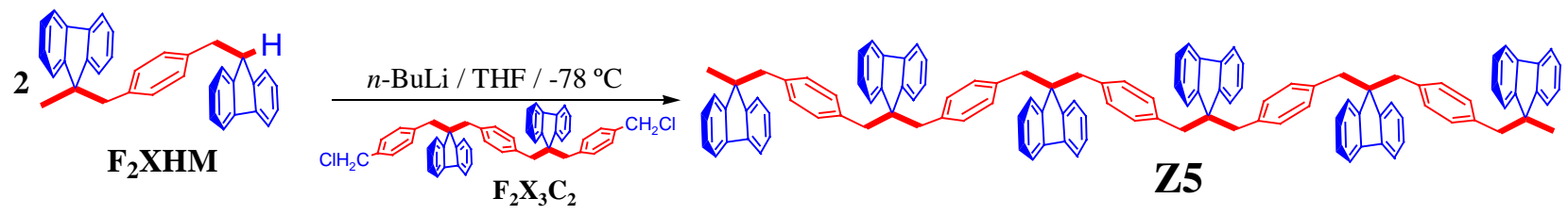

To a solution of $\mathbf{F}_{2} \mathbf{X H M}(1.26 \mathrm{~g}, 2.82 \mathrm{mmol})$ in anhydrous tetrahydrofuran $(20 \mathrm{~mL})$ under an argon atmosphere and cooled to $-78{ }^{\circ} \mathrm{C}, n$-butyllithium $(2.5 \mathrm{M}$ in hexanes, $1.1 \mathrm{~mL})$ was added dropwise. After $10 \mathrm{~min}$. of stirring, solid $\mathbf{F}_{2} \mathbf{X}_{3} \mathbf{C}_{2}(1.0 \mathrm{~g}, 1.4 \mathrm{mmol})$ was added and the mixture was stirred for $15 \mathrm{~min}$. at $-78{ }^{\circ} \mathrm{C}$. The cooling bath was removed and the reaction mixture was stirred for an additional $1 \mathrm{~h}$. It was then poured onto water $(100 \mathrm{~mL})$ and the aqueous layer was extracted with dichloromethane $(3 \quad x \quad 100 \quad \mathrm{~mL})$. The combined dichloromethane extracts were dried over anhydrous magnesium sulfate, filtered, and evaporated under reduced pressure to afford a pale-yellow solid. The crude material was purified by flash chromatography on silica gel using $15 \%$ ethyl acetate/hexanes as an eluent to afford $\mathbf{Z 5}$ as a white solid. Yield: $1.9 \mathrm{~g}(89 \%)$; mp 230-232 ${ }^{\circ} \mathrm{C} ;{ }^{1} \mathrm{H}$ NMR $\left(\mathrm{CDCl}_{3}\right) \delta: 1.30(\mathrm{~s}, 6 \mathrm{H}), 2.78(\mathrm{~s}, 4 \mathrm{H})$, 
$2.978(\mathrm{~m}, 16 \mathrm{H}), 6.20(\mathrm{~m}, 20 \mathrm{H}) 6.72(\mathrm{~m}, 12 \mathrm{H}), 7.14(\mathrm{~m}, 20 \mathrm{H}), 7.33(\mathrm{~m}, 10 \mathrm{H}), 7.52(\mathrm{~d}, 6 \mathrm{H}) ;{ }^{13} \mathrm{C}$ $\operatorname{NMR}\left(\mathrm{CDCl}_{3}\right) \delta: 24.63,25.89,44.69,45.00,45.59,51.38,56.76,56.67,68.18,119.41,119.78$ $123.83,124.84,126.12,126.70,126.85,126.94,129.05,134.69,134.75,134.89,135.05,139.76$, $140.71,140.79,148.48,151.37$.

\section{Synthesis of Z6.}
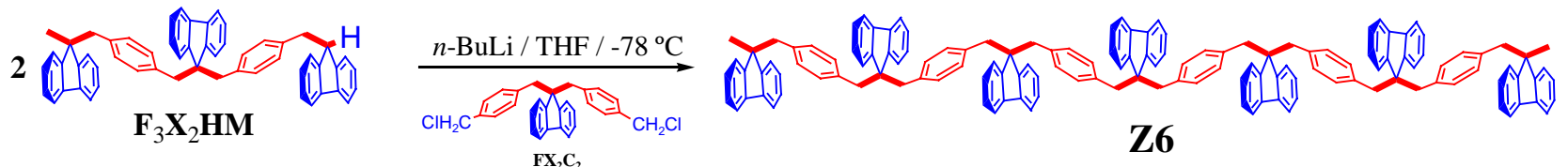

A solution of $\mathbf{F}_{3} \mathbf{X}_{2} \mathbf{H M}(1.6 \mathrm{~g}, 2.2 \mathrm{mmol})$ in anhydrous tetrahydrofuran $(15 \mathrm{~mL})$ at $-78{ }^{\circ} \mathrm{C}$ was reacted with $n$-butyllithium ( $2.5 \mathrm{M}$ in hexanes, $0.88 \mathrm{~mL})$ and solid $\mathbf{F X}_{\mathbf{2}} \mathbf{C}_{\mathbf{2}}(0.5 \mathrm{~g}, 1.1 \mathrm{mmol})$ as described above to afford a pale-yellow solid material. The crude material was purified by flash chromatography on silica gel using 20\% ethyl acetate/hexanes as an eluent to afford $\mathbf{Z 6}$ as a white solid. Yield: $2.0 \mathrm{~g}(50 \%)$; mp 180-182 ${ }^{\circ} \mathrm{C} ;{ }^{1} \mathrm{H}$ NMR $\left(\mathrm{CDCl}_{3}\right) \delta: 1.35$ (s, 6H), $2.82(\mathrm{~s}, 4 \mathrm{H})$, $3.00(\mathrm{~m}, 20 \mathrm{H}), 6.24(\mathrm{~m}, 24 \mathrm{H}) 6.97(\mathrm{~m}, 12 \mathrm{H}), 7.19(\mathrm{~m}, 30 \mathrm{H}), 7.38(\mathrm{~m}, 10 \mathrm{H}), 7.55(\mathrm{~d}, 4 \mathrm{H}) ;{ }^{13} \mathrm{C}$ $\operatorname{NMR}\left(\mathrm{CDCl}_{3}\right) \delta: 23.24,43.35,45.19,49.99,55.17,55.25,118.03,118.39,122.46,123.51$, $124.74,125.33,125.45,125.57,127.67,133.31,139.31,139.40,147.09,149.92$.

\section{Synthesis of Z7.}

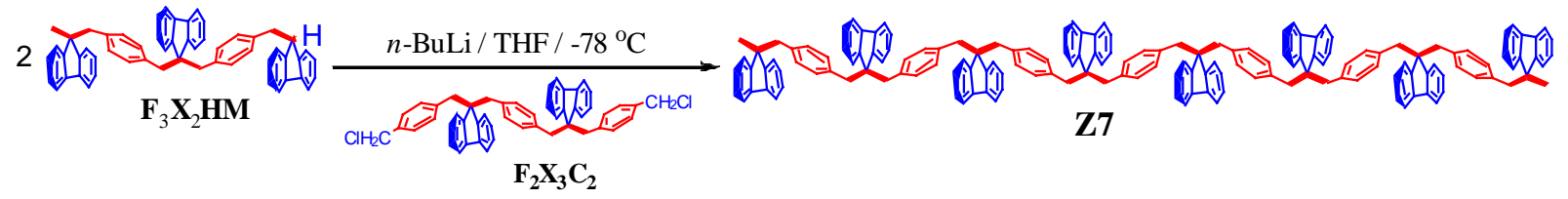

A solution of $\mathbf{F}_{3} \mathbf{X}_{2} \mathbf{H M}(1.4 \mathrm{~g}, 2.0 \mathrm{mmol})$ in anhydrous tetrahydrofuran $(15 \mathrm{~mL})$ at $-78{ }^{\circ} \mathrm{C}$ was reacted with $n$-butyllithium $(2.5 \mathrm{M}$ in hexanes, $0.80 \mathrm{~mL})$ and solid $\mathbf{F}_{2} \mathbf{X}_{\mathbf{3}} \mathbf{C}_{2}(0.71 \mathrm{~g}, 1.0$ mmol) as described above to afford a white solid. The crude material was purified by flash 
chromatography on silica gel using $20 \%$ ethyl acetate/hexanes as an eluent to afford $\mathbf{Z 7}$ as a white solid. Yield: $1.9 \mathrm{~g}(46 \%)$; m 209-211 ${ }^{\circ} \mathrm{C} ;{ }^{1} \mathrm{H} \mathrm{NMR}\left(\mathrm{CDCl}_{3}\right) \delta: 1.38(\mathrm{~s}, 6 \mathrm{H}), 2.83(\mathrm{~s}, 4 \mathrm{H})$, $3.00(\mathrm{~m}, 24 \mathrm{H}), 6.24(\mathrm{~m}, 28 \mathrm{H}) 6.97(\mathrm{~m}, 16 \mathrm{H}), 7.19(\mathrm{~m}, 34 \mathrm{H}), 7.38(\mathrm{~m}, 10 \mathrm{H}), 7.56(\mathrm{~d}, 4 \mathrm{H}) ;{ }^{13} \mathrm{C}$ $\operatorname{NMR}\left(\mathrm{CDCl}_{3}\right) \delta: 24.63,44.72,44.98,46.58,51.37,56.53,56.55,56.66,119.40,119.75,123.82$, $124.87,126.11,126.70,126.81,126.93,129.05,134.68,134.73,134.88,135.05,139.75,140.69$, $140.77,148.47,151.30$.

\section{Synthesis of Z8.}

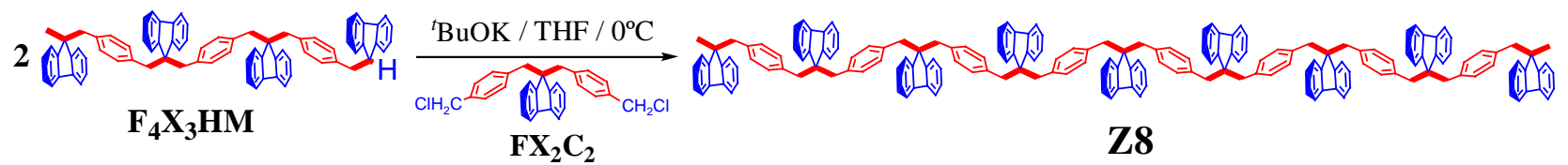

To a solution of $\mathbf{F}_{\mathbf{4}} \mathbf{X}_{\mathbf{3}} \mathbf{H M}(0.25 \mathrm{~g}, 0.25 \mathrm{mmol})$ in anhydrous tetrahydrofuran $(20 \mathrm{ml})$ at $\sim 0$ ${ }^{\circ} \mathrm{C}$, potassium tert-butoxide $(0.12 \mathrm{~g}, 1.0 \mathrm{mmol})$ was added. After $10 \mathrm{~min}$. of stirring, solid $\mathbf{F X}_{2} \mathbf{C}_{2}(0.06 \mathrm{~g}, 0.13 \mathrm{mmol})$ was added and the mixture was stirred for $15 \mathrm{~min}$. at $\sim 0{ }^{\circ} \mathrm{C}$. The cooling bath was removed and the reaction mixture was stirred for $12 \mathrm{~h}$. It was then poured onto water $(100 \mathrm{~mL})$ and the aqueous layer was extracted with dichloromethane $(3 \times 100 \mathrm{~mL})$. The combined dichloromethane extracts were dried over anhydrous magnesium sulfate, filtered, and evaporated under reduced pressure to afford a yellow solid. The crude material was purified by flash chromatography on silica gel using 5\% ethyl acetate/hexanes as an eluent to afford $\mathbf{Z 8}$ as a white solid. Yield: $0.4 \mathrm{~g}$ (68\%); mp 215-217 ${ }^{\circ} \mathrm{C} ;{ }^{1} \mathrm{H}$ NMR $\left(\mathrm{CDCl}_{3}\right) \delta: 1.36$ (s, 6H), 2.83 (s, 4H), $3.00(\mathrm{~m}, 28 \mathrm{H}), 6.24(\mathrm{~m}, 32 \mathrm{H}) 6.95(\mathrm{~m}, 16 \mathrm{H}), 7.19(\mathrm{~m}, 42 \mathrm{H}), 7.39(\mathrm{~m}, 10 \mathrm{H}), 7.56(\mathrm{~d}, 4 \mathrm{H}) ;{ }^{13} \mathrm{C}$ $\operatorname{NMR}\left(\mathrm{CDCl}_{3}\right) \delta: 24.75,44.81,44.84,46.71,51.50,51.52,56.65,56.68,56.79,119.51,119.87$, $123.95,125.02,126.22,126.24,126.82,126.93,126.96,127.05,129.15,129.23,134.79,134.86$ $135.00,135.14,139.87,140.80,140.89,148.58,151.41$. 


\section{Synthesis of Z9.}

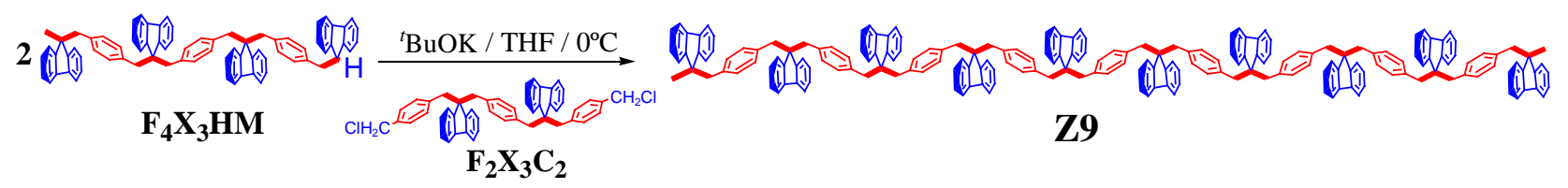

A solution of $\mathbf{F}_{\mathbf{4}} \mathbf{X}_{\mathbf{3}} \mathbf{H M}(0.25 \mathrm{~g}, 0.25 \mathrm{mmol})$ in anhydrous tetrahydrofuran $(20 \mathrm{ml})$ at $0{ }^{\circ} \mathrm{C}$ was reacted with potassium tert-butoxide $(0.12 \mathrm{~g}, 1.0 \mathrm{mmol})$ and $\mathbf{F}_{\mathbf{2}} \mathbf{X}_{\mathbf{3}} \mathbf{C}_{\mathbf{2}}(0.1 \mathrm{~g}, 0.13 \mathrm{mmol})$ as described above to afford a yellow solid. The crude material was purified by flash chromatography on silica gel using 5\% ethyl acetate/hexanes as an eluent to afford a white solid. Yield: $0.5 \mathrm{~g}$ (77\%); mp 220-222 ${ }^{\circ} \mathrm{C} ;{ }^{1} \mathrm{H}$ NMP $\left(\mathrm{CDCl}_{3}\right) \delta: 1.35$ (s, 6H), 2.82 (s, 4H), 3.00 (m, 32H), $6.24(\mathrm{~m}, 36 \mathrm{H}) 6.97(\mathrm{~m}, 24 \mathrm{H}), 7.19(\mathrm{~m}, 42 \mathrm{H}), 7.38(\mathrm{~m}, 10 \mathrm{H}), 7.55(\mathrm{~d}, 4 \mathrm{H}) ;{ }^{13} \mathrm{C}$ NMR $\left(\mathrm{CDCl}_{3}\right) \delta: 24.62,44.69,51.36,56.51,56.54,56.65,119.51,119.86,123.94,124.00,126.21$, $126.24,126.82,126.92,127.05,129.14,129.23,134.81,134.88,135.02,135.18,139.89,140.81$, 140.84, 140.92, 148.63, 151.42. 


\section{${ }^{1} \mathrm{H}$ NMR spectrum of FXEM}

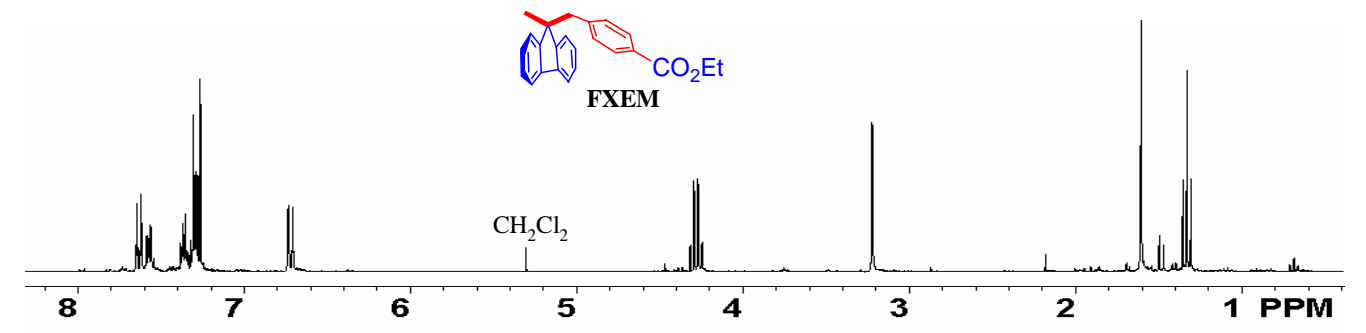

\section{${ }^{13} \mathrm{C}$ NMR spectrum of FXEM}

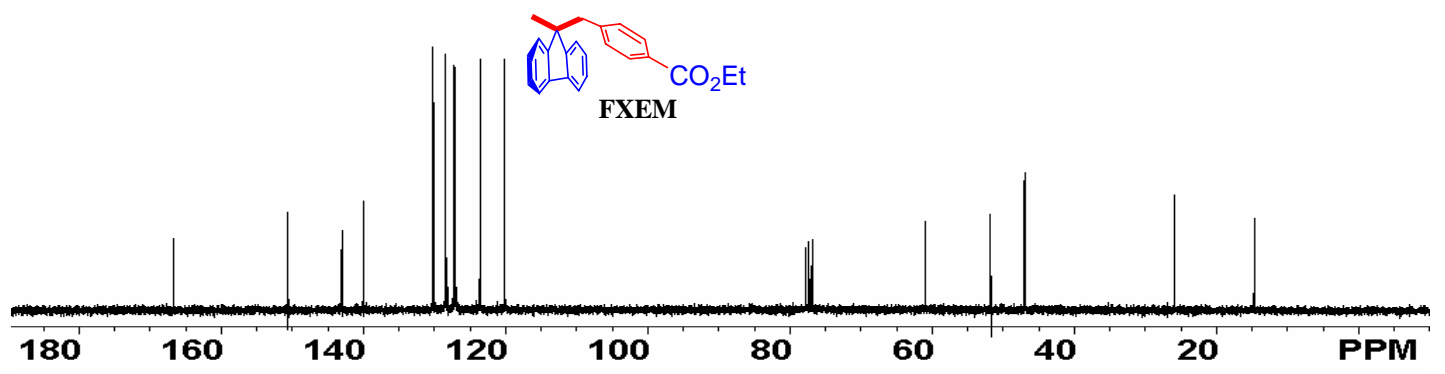

\section{${ }^{1} \mathrm{H}$ NMR spectram of FXAM FXAM}

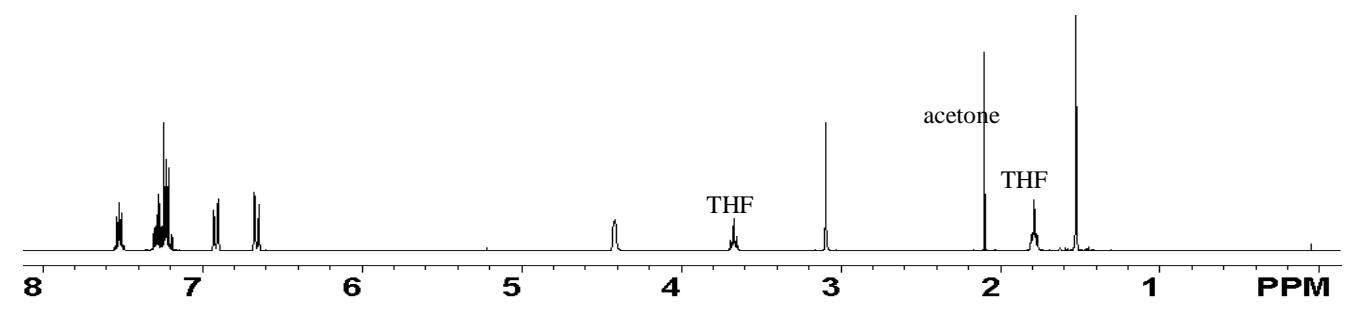




\section{${ }^{13} \mathrm{C}$ NMR spectrum of FXAM}

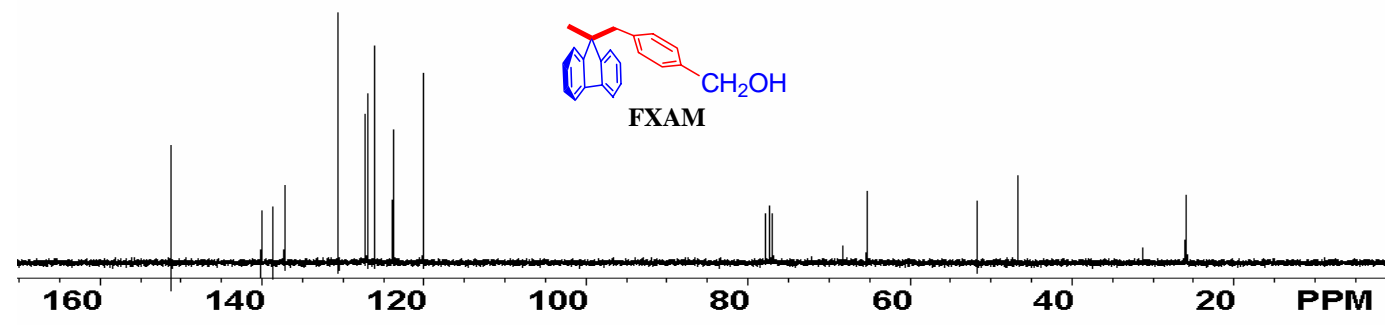

${ }^{1} \mathrm{H}$ NMR spectrum of $\mathbf{F X C M}$

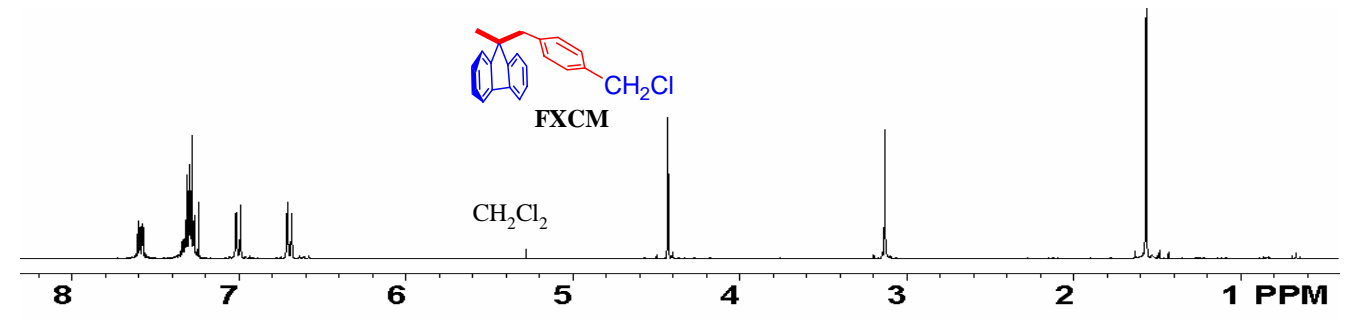

\section{${ }^{13} \mathrm{C}$ NMR spectrum of FXCM}

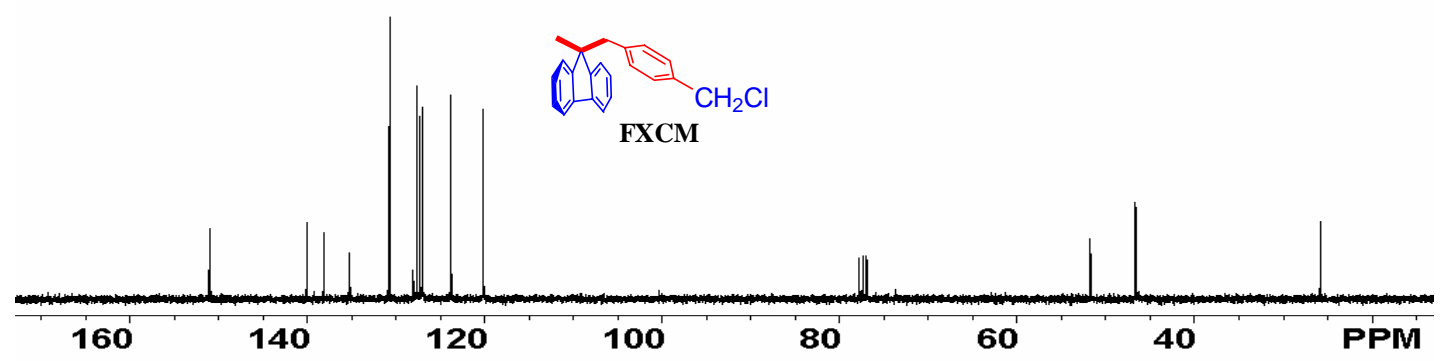




\section{${ }^{1} \mathrm{H}$ NMR spectrum of $\mathbf{F}_{\mathbf{2}} \mathbf{X H M}$}

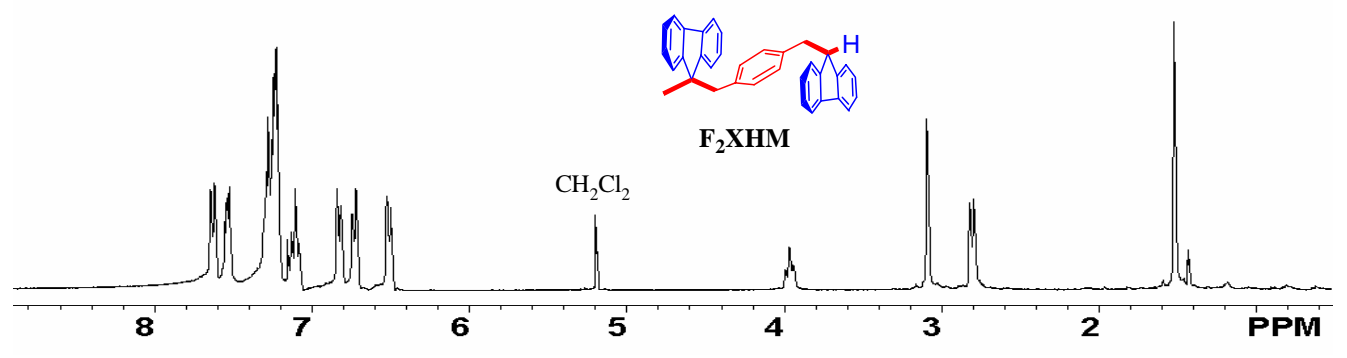

${ }^{13} \mathrm{C}$ NMR spectrum of $\mathbf{F}_{\mathbf{2}} \mathbf{X H M}$

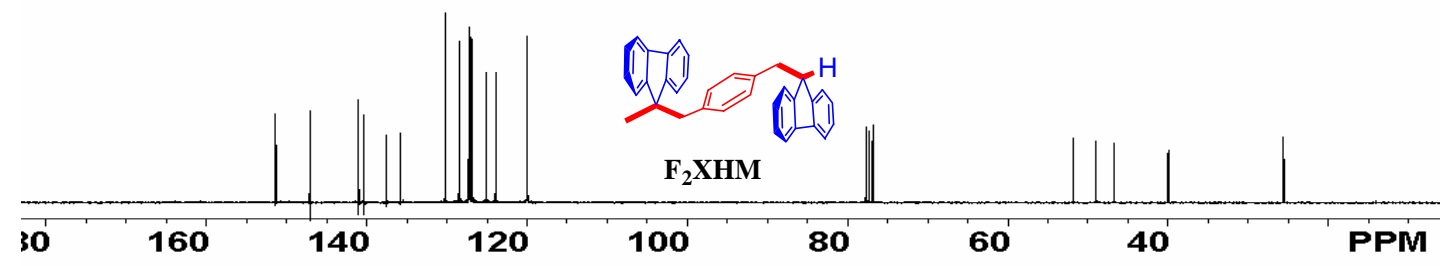

${ }^{1} \mathrm{H}$ NMR spectrum of $\mathbf{F}_{\mathbf{2}} \mathbf{X}_{\mathbf{2}} \mathbf{E M}$

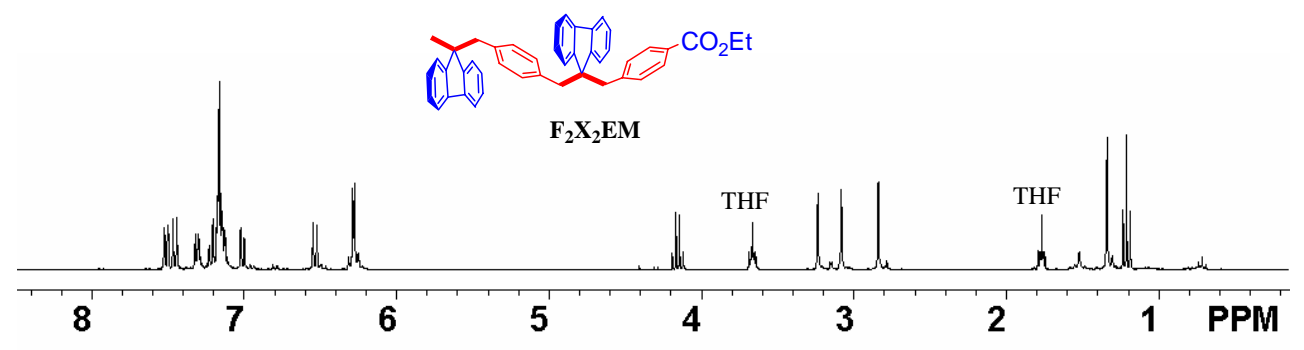




\section{${ }^{13} \mathrm{C}$ NMR spectrum of $\mathbf{F}_{\mathbf{2}} \mathbf{X}_{\mathbf{2}} \mathbf{E M}$}

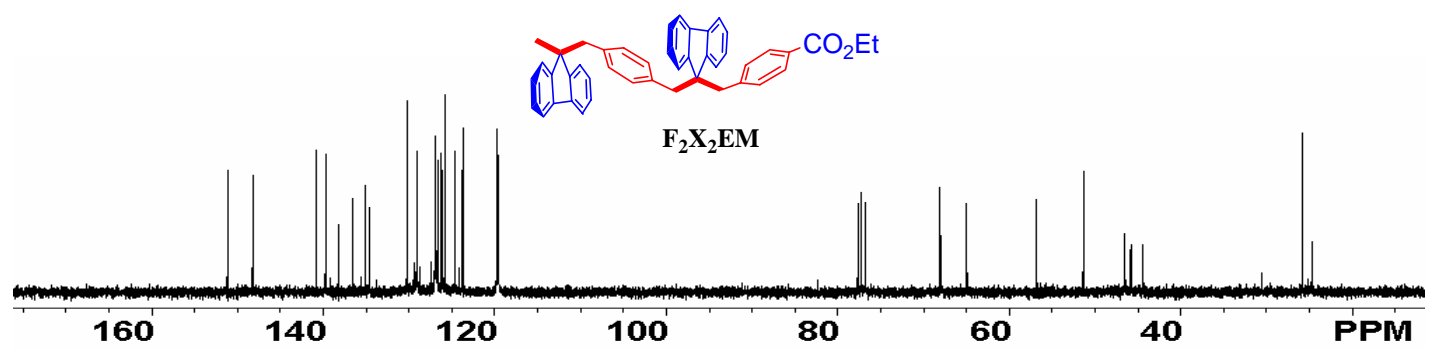

${ }^{1} \mathrm{H}$ NMR spectrum of $\mathbf{F}_{\mathbf{2}} \mathbf{X}_{\mathbf{2}} \mathbf{A M}$ (crude)

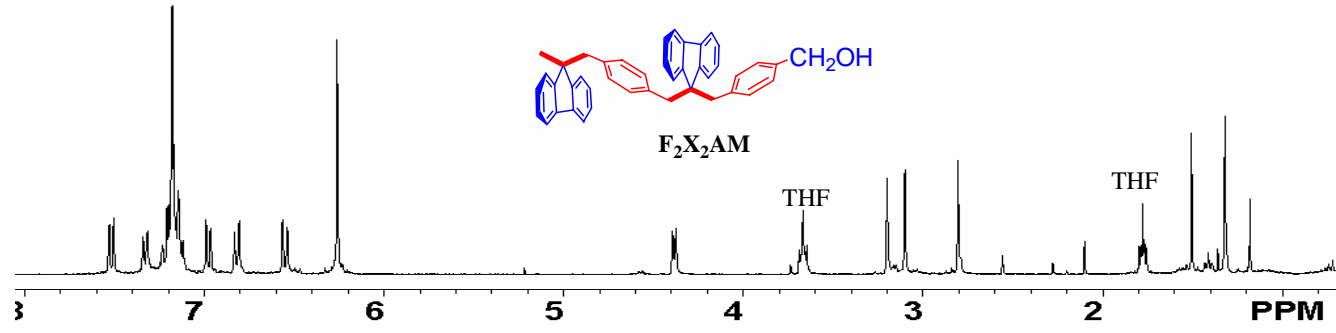

${ }^{13} \mathrm{C}$ NMR spectrum of $\mathbf{F}_{\mathbf{2}} \mathbf{X}_{\mathbf{2}} \mathbf{A M}$

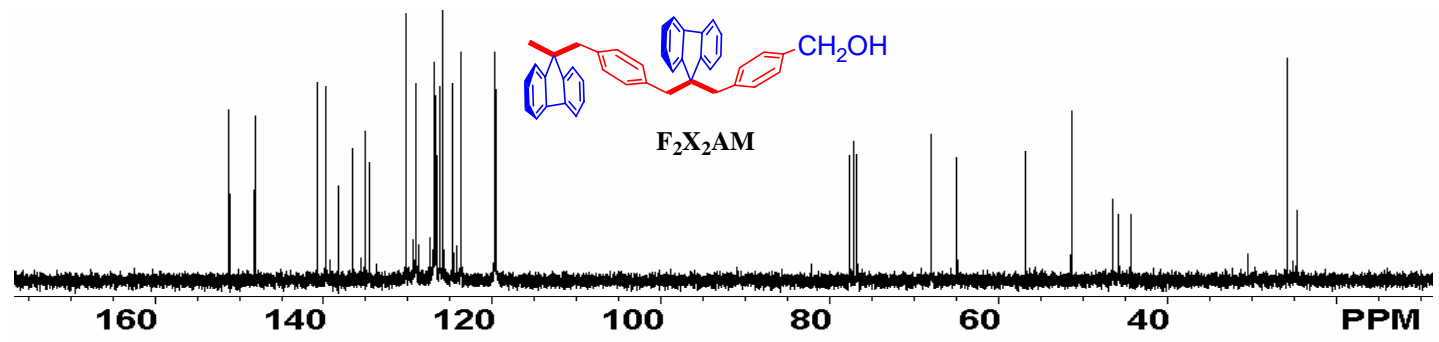




\section{${ }^{1} \mathrm{H}$ NMR spectrum of $\mathbf{F}_{\mathbf{2}} \mathbf{X}_{\mathbf{2}} \mathbf{C M}$}

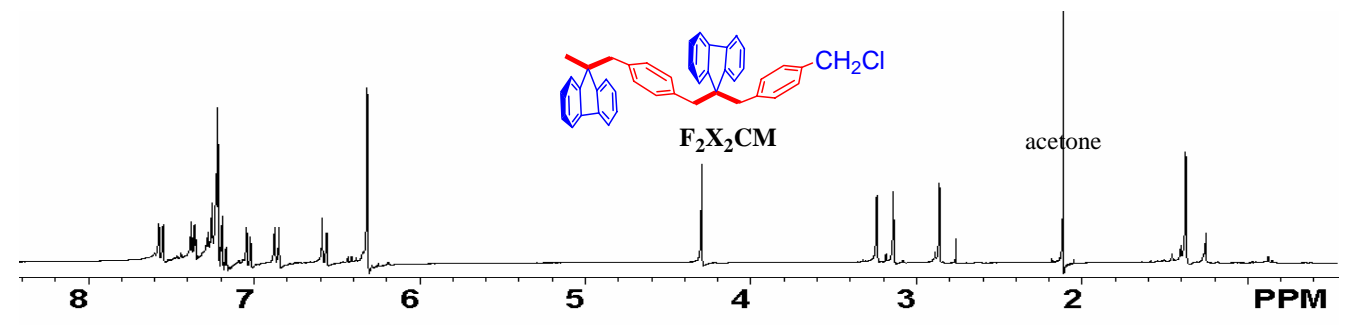

\section{${ }^{13} \mathrm{C}$ NMR spectrum of $\mathbf{F}_{\mathbf{2}} \mathbf{X}_{\mathbf{2}} \mathbf{C M}$}

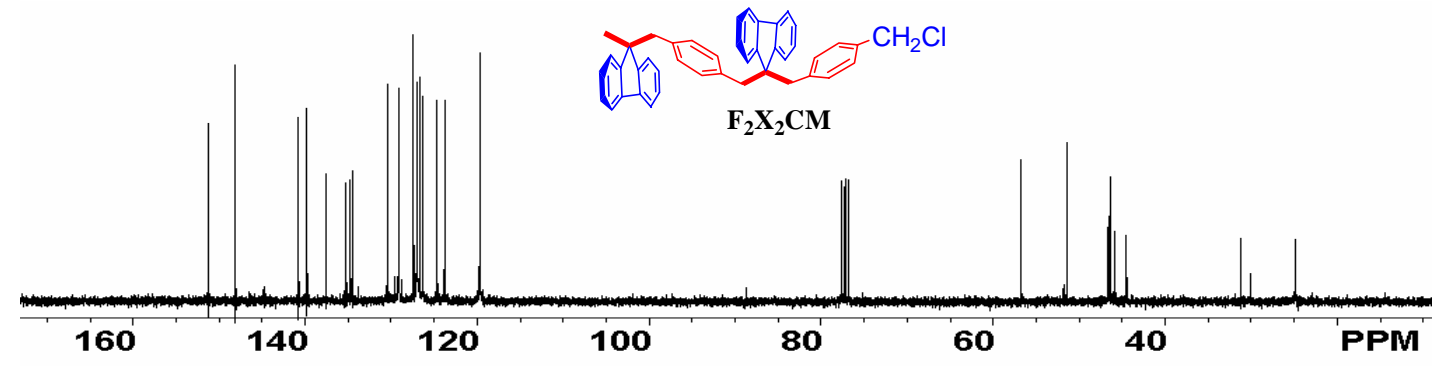

${ }^{1} \mathrm{H}$ NMR spectrum of $\mathbf{F}_{\mathbf{3}} \mathbf{X}_{\mathbf{2}} \mathbf{H M}$ (crude)

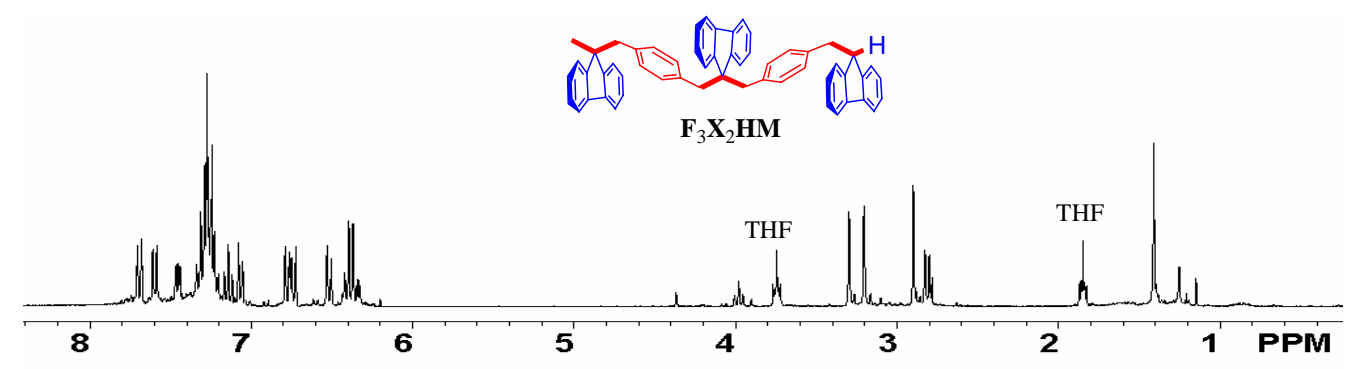




\section{${ }^{13} \mathrm{C}$ NMR spectrum of $\mathbf{F}_{\mathbf{3}} \mathbf{X}_{\mathbf{2}} \mathbf{H M}$}

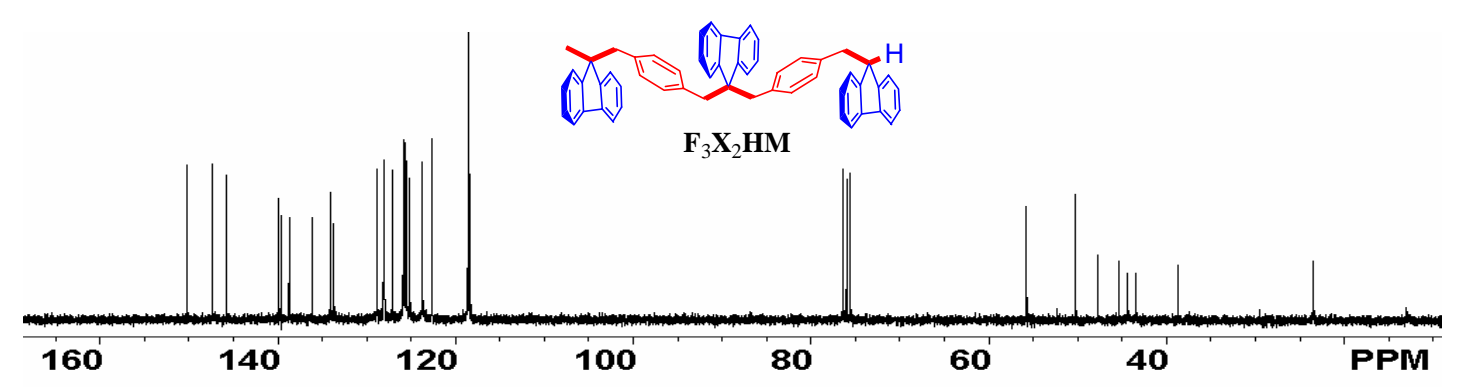

${ }^{1} \mathrm{H}$ NMR spectrum of $\mathbf{F}_{\mathbf{3}} \mathbf{X}_{\mathbf{2}} \mathbf{E M}$

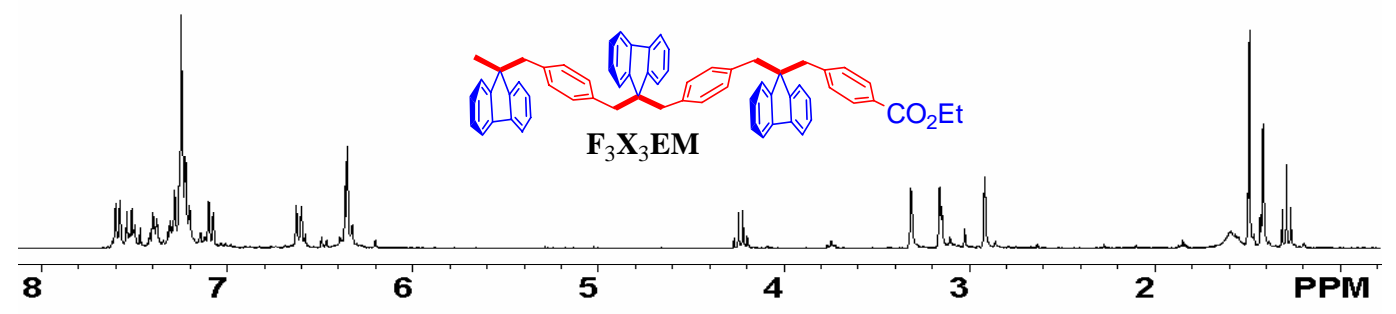

${ }^{13} \mathrm{C}$ NMR spectrum of $\mathbf{F}_{\mathbf{3}} \mathbf{X}_{\mathbf{2}} \mathbf{E M}$

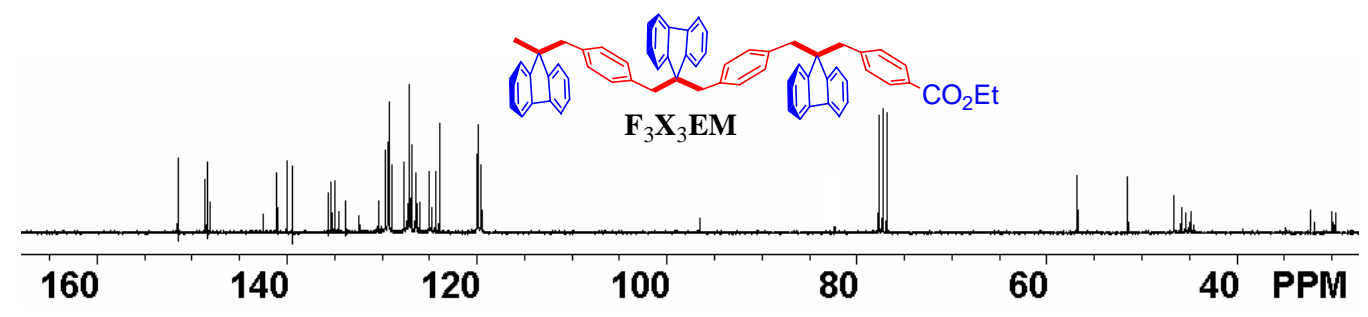




\section{${ }^{1} \mathrm{H}$ NMR spectrum of $\mathbf{F}_{\mathbf{3}} \mathbf{X}_{\mathbf{3}} \mathbf{A M}$ (crude)}

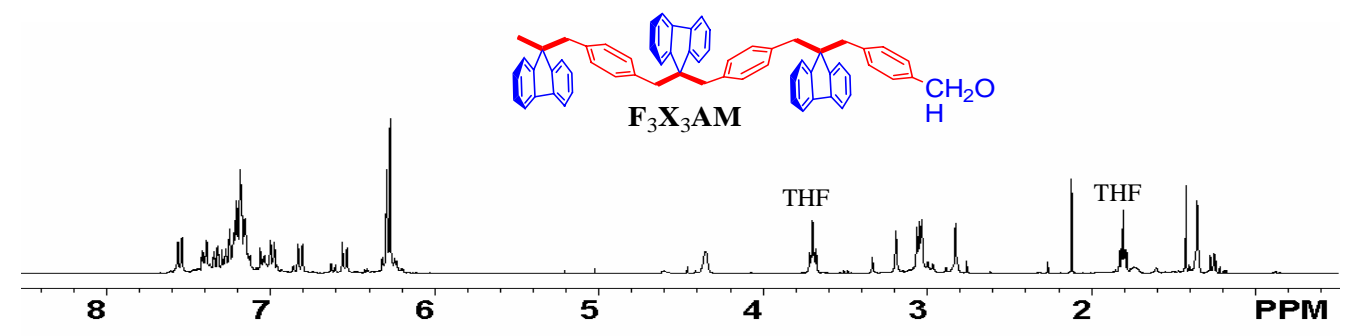

\section{${ }^{13} \mathrm{C}$ NMR spectrum of $\mathbf{F}_{\mathbf{3}} \mathbf{X}_{\mathbf{3}} \mathbf{A M}$}

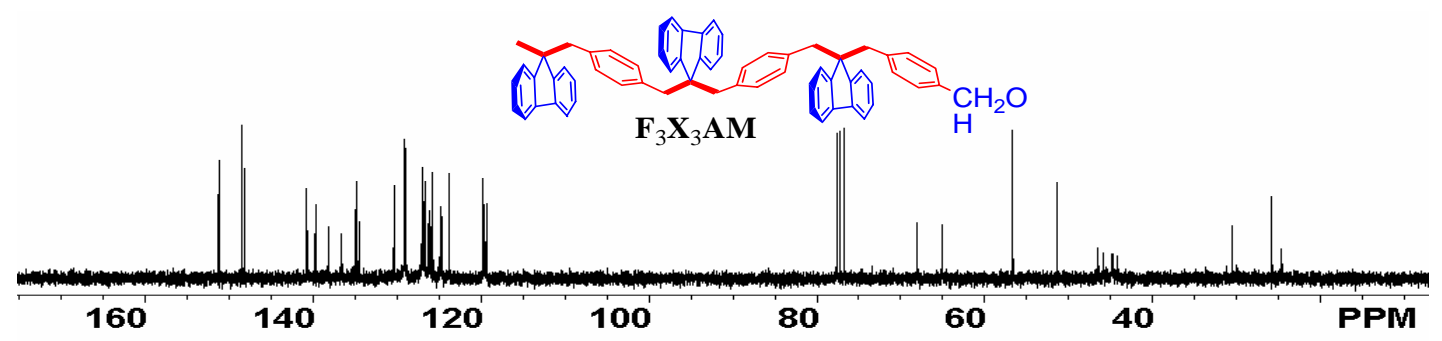

\section{${ }^{1} \mathrm{H}$ NMR spectrum of $\mathbf{F}_{\mathbf{3}} \mathbf{X}_{\mathbf{3}} \mathbf{C M}$}

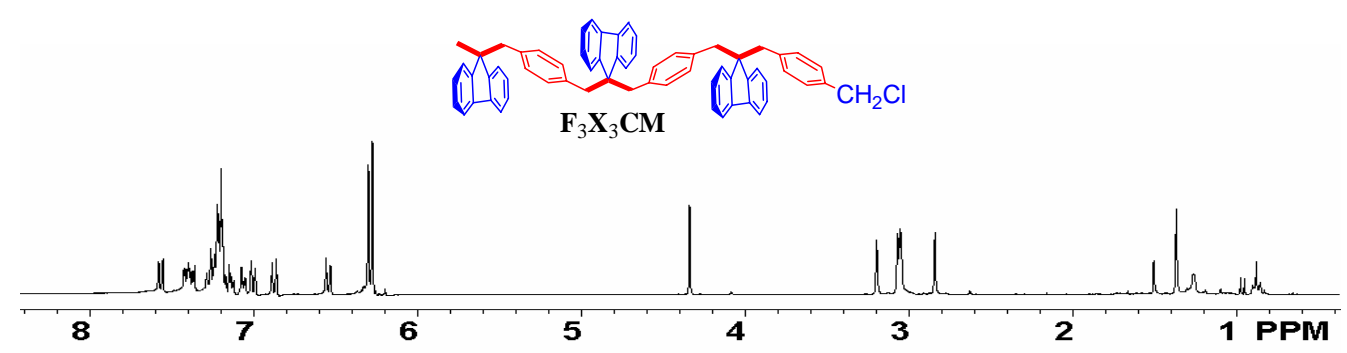




\section{${ }^{13} \mathrm{C}$ NMR spectrum of $\mathbf{F}_{\mathbf{3}} \mathbf{X}_{\mathbf{3}} \mathbf{C M}$}

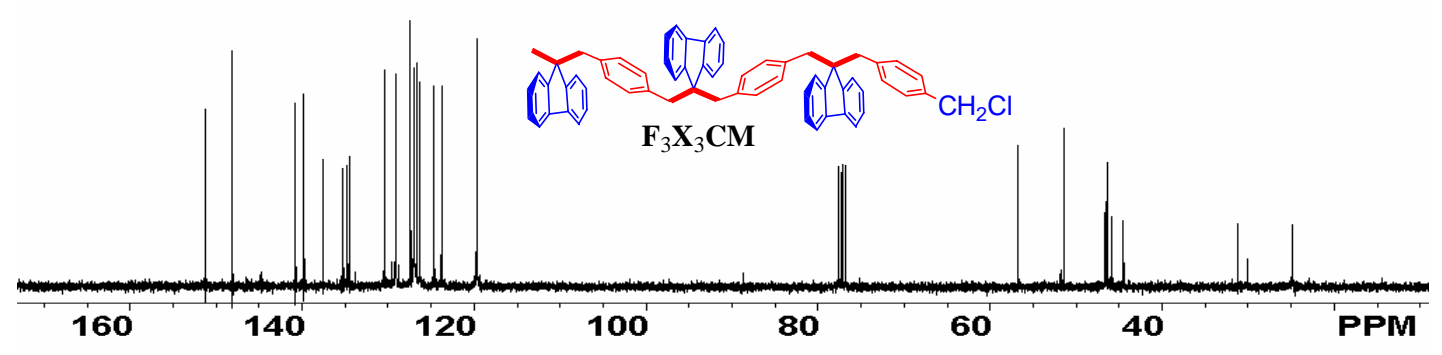

\section{${ }^{1} \mathrm{H}$ NMR spectrum of $\mathbf{F}_{\mathbf{4}} \mathbf{X}_{\mathbf{3}} \mathbf{H M}$}

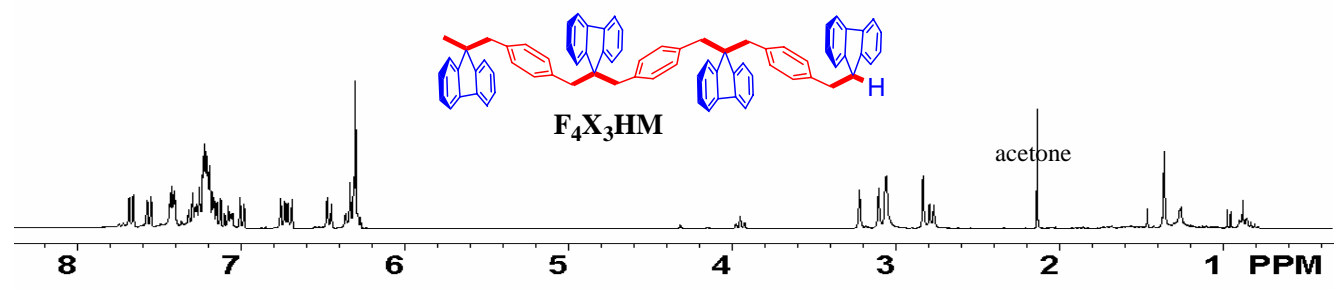

\section{${ }^{13} \mathrm{C}$ NMR spectrum of $\mathbf{F}_{\mathbf{4}} \mathbf{X}_{\mathbf{3}} \mathbf{H M}$}

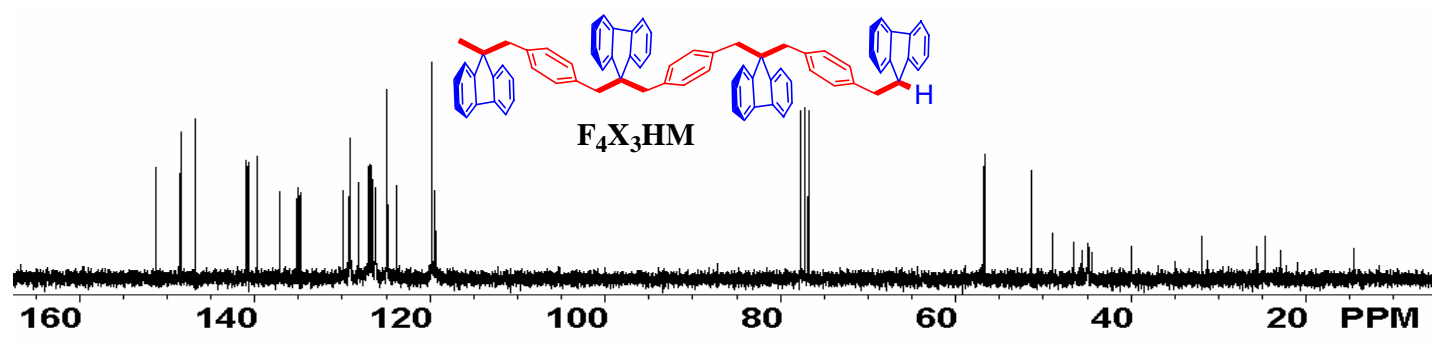




\section{${ }^{1} \mathrm{H}$ NMR spectrum of $\mathbf{F} \mathbf{X}_{\mathbf{2}} \mathbf{E}_{\mathbf{2}}$}
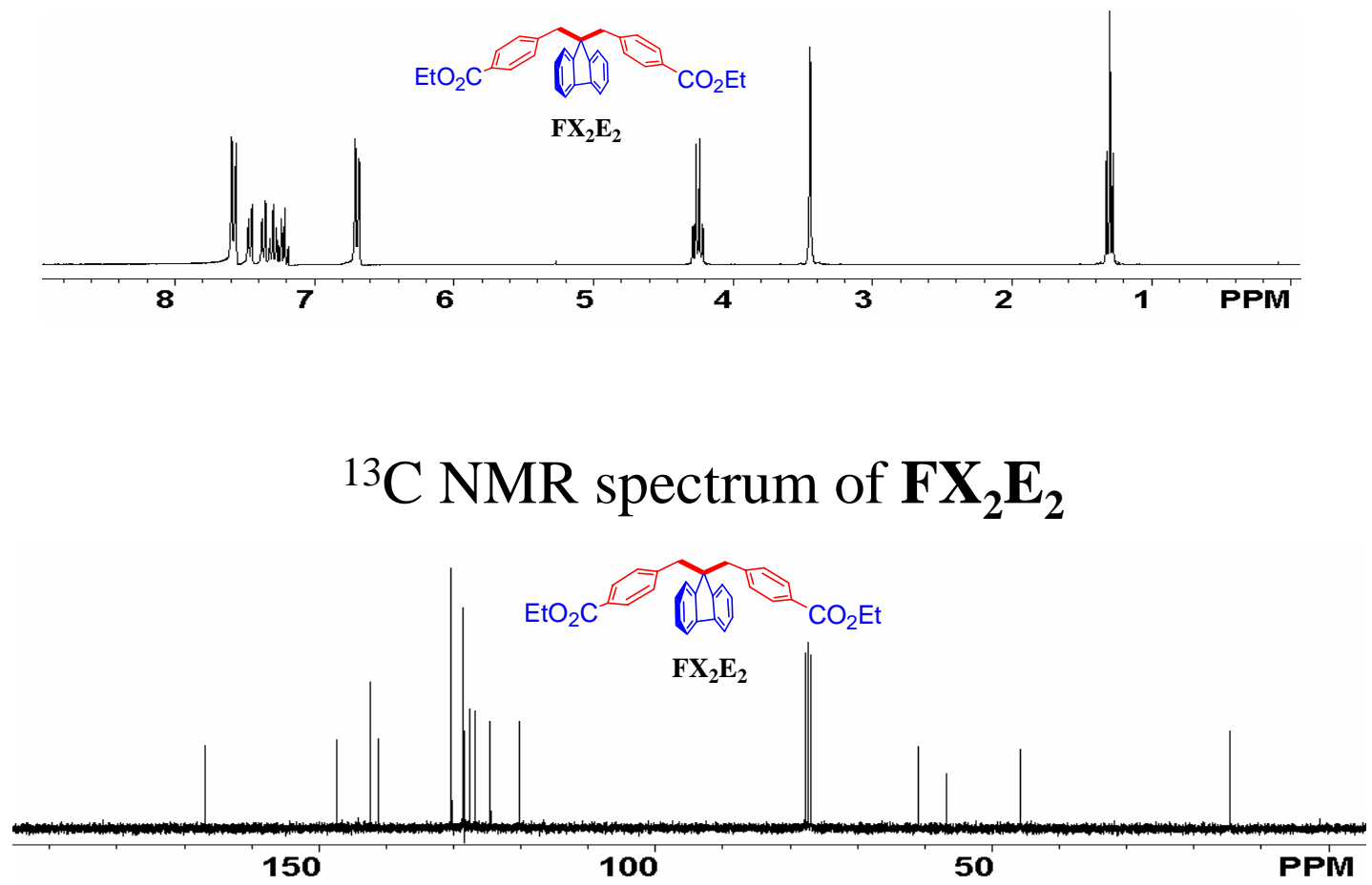

${ }^{1} \mathrm{H}$ NMR spectrum of $\mathbf{F} \mathbf{X}_{\mathbf{2}} \mathbf{A}_{\mathbf{2}}$

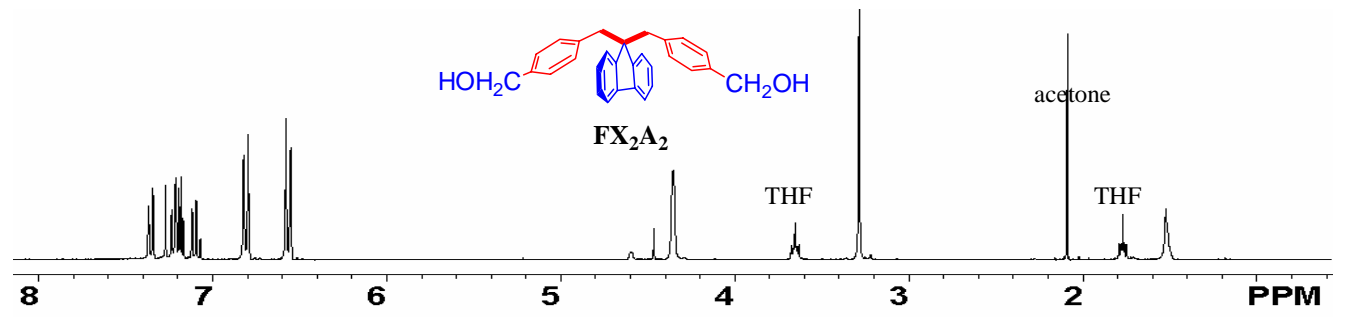

\section{${ }^{13} \mathrm{C}$ NMR spectrum of $\mathbf{F} \mathbf{X}_{\mathbf{2}} \mathbf{A}_{\mathbf{2}}$}

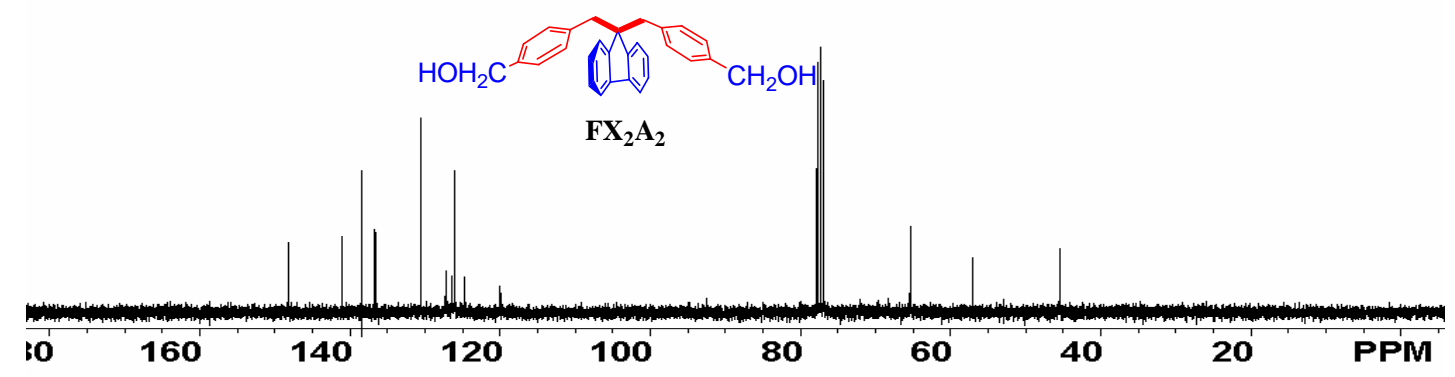




\section{${ }^{1} \mathrm{H}$ NMR spectrum of $\mathbf{F} \mathbf{X}_{\mathbf{2}} \mathbf{C}_{\mathbf{2}}$}

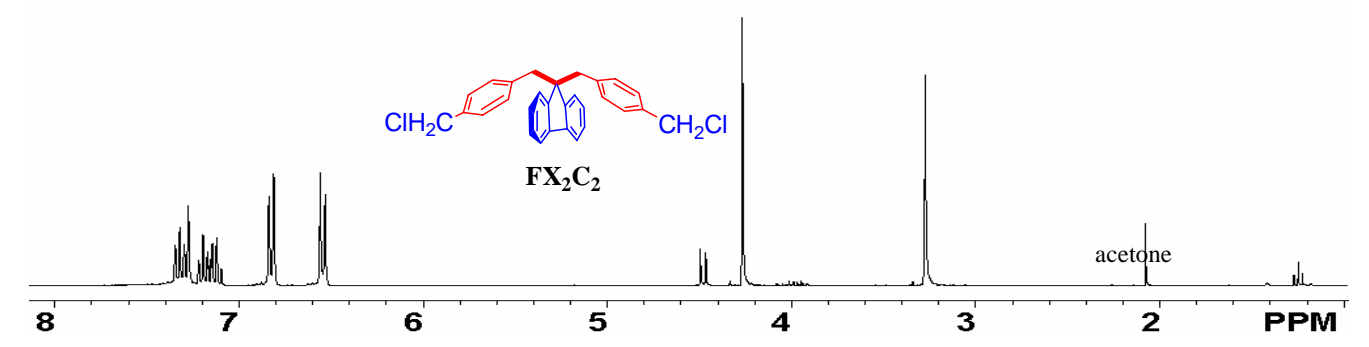

\section{${ }^{13} \mathrm{C}$ NMR spectrum of $\mathbf{F} \mathbf{X}_{\mathbf{2}} \mathbf{C}_{\mathbf{2}}$}

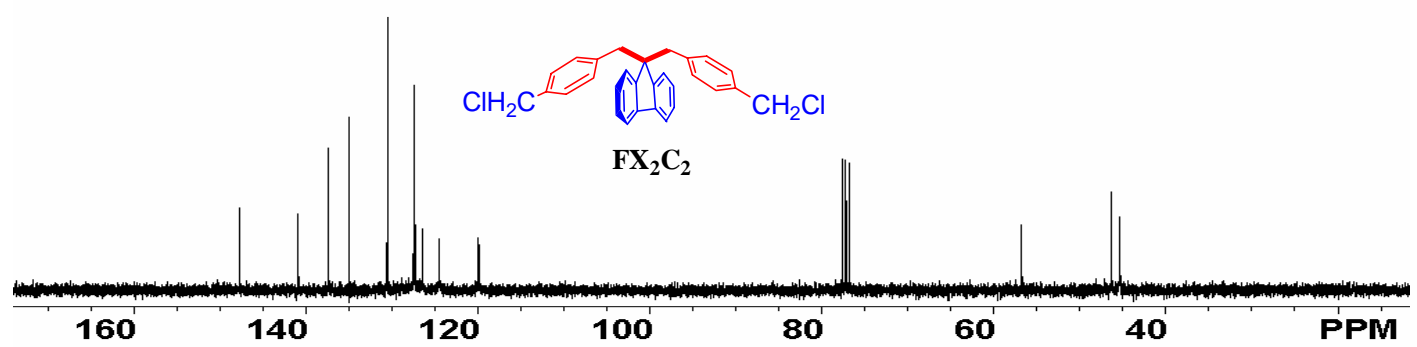

\section{${ }^{1} \mathrm{H}$ NMR spectrum of $\mathbf{F}_{\mathbf{2}} \mathbf{X} \mathbf{H}_{\mathbf{2}}$}

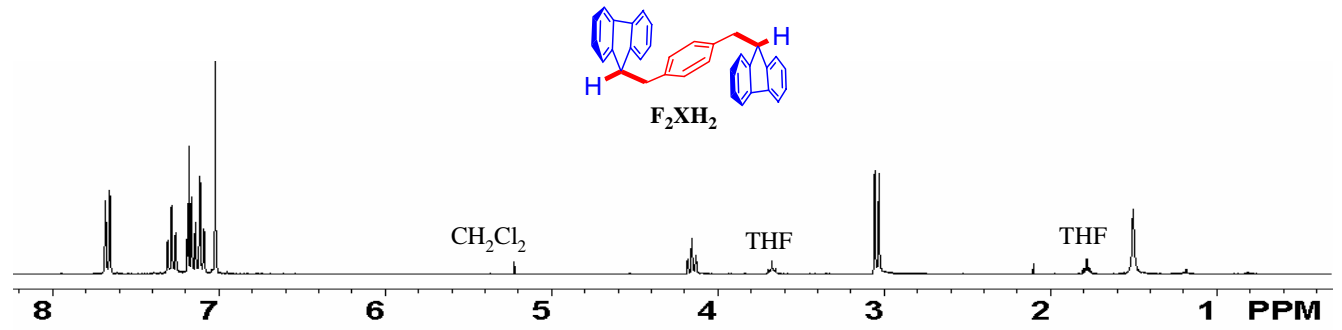




\section{${ }^{13} \mathrm{C}$ NMR spectrum of $\mathbf{F}_{\mathbf{2}} \mathbf{X H}_{\mathbf{2}}$}

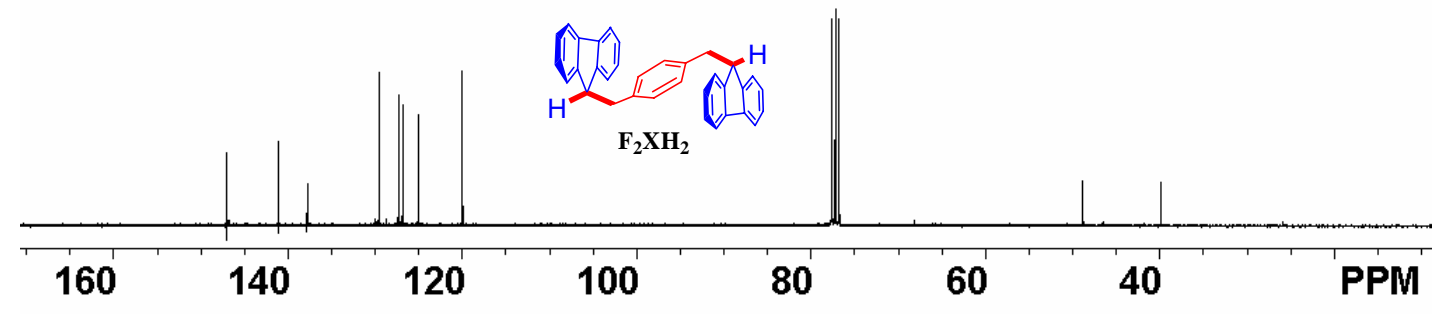

\section{${ }^{1} \mathrm{H}$ NMR spectrum of $\mathbf{F}_{\mathbf{2}} \mathbf{X}_{\mathbf{3}} \mathbf{E}_{\mathbf{2}}$}

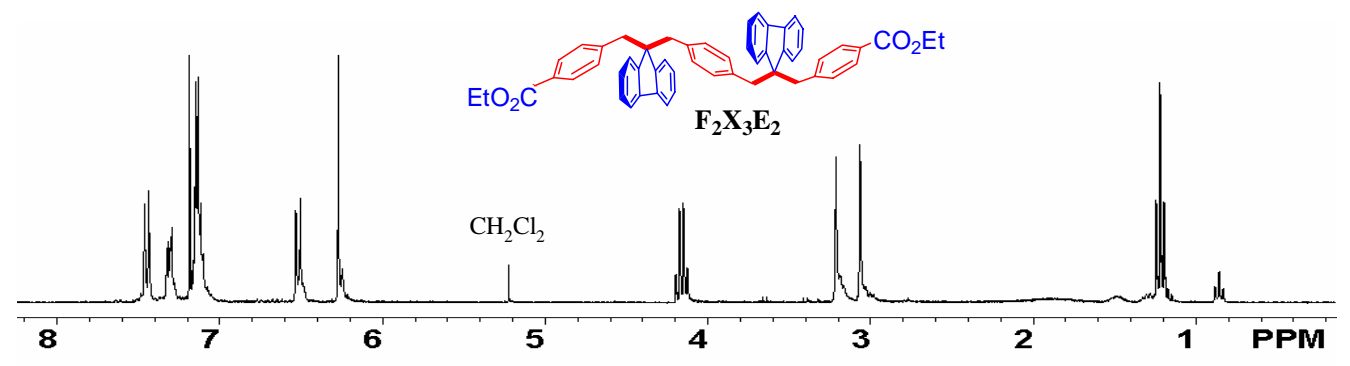

\section{${ }^{13} \mathrm{C}$ NMR spectrum of $\mathbf{F}_{\mathbf{2}} \mathbf{X}_{\mathbf{3}} \mathbf{E}_{\mathbf{2}}$}

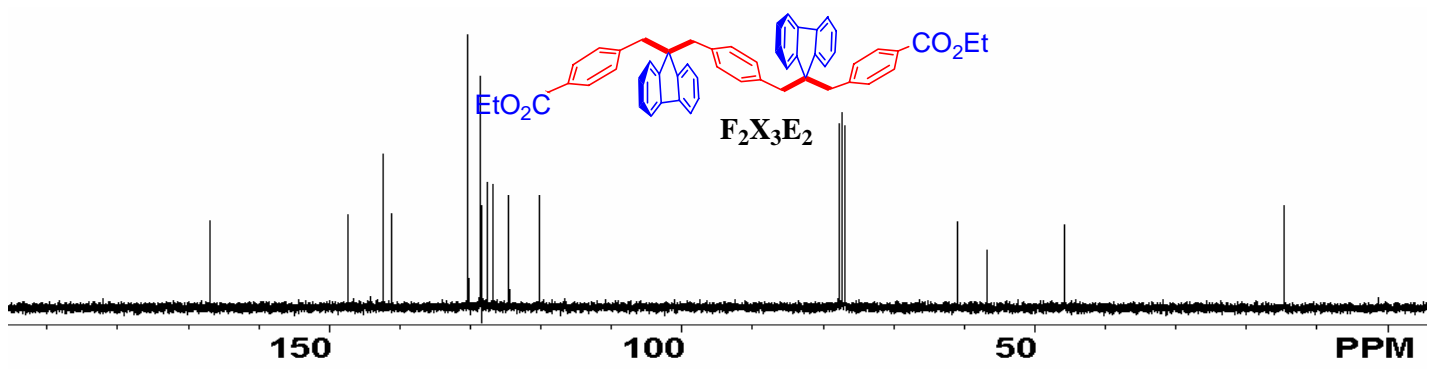




\section{${ }^{1} \mathrm{H}$ NMR spectrum of $\mathbf{F}_{\mathbf{2}} \mathbf{X}_{\mathbf{3}} \mathbf{A}_{\mathbf{2}}$}

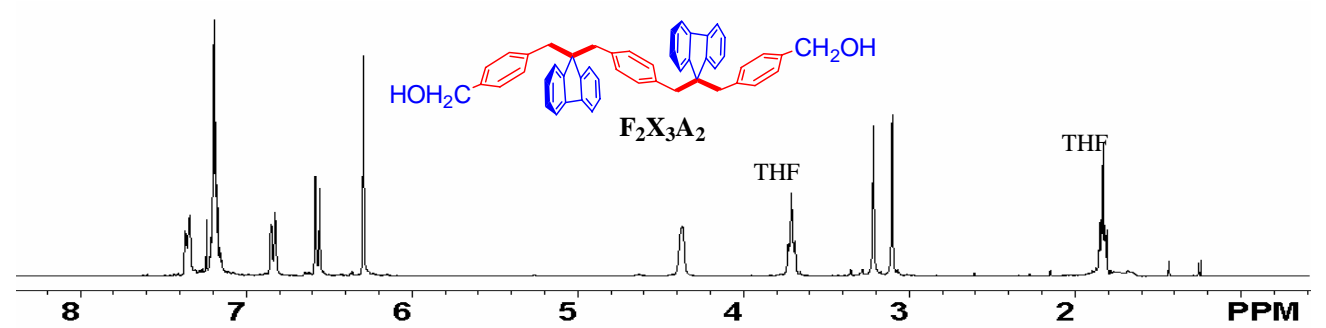

\section{${ }^{13} \mathrm{C}$ NMR spectrum of $\mathbf{F}_{\mathbf{2}} \mathbf{X}_{\mathbf{3}} \mathbf{A}_{\mathbf{2}}$}

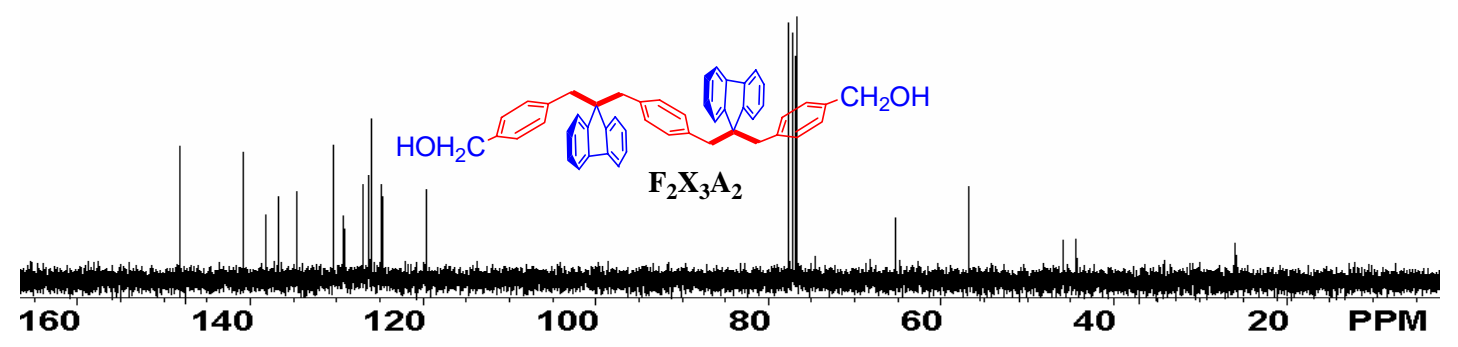

${ }^{1} \mathrm{H}$ NMR spectrum of $\mathbf{F}_{\mathbf{2}} \mathbf{X}_{\mathbf{3}} \mathbf{C}_{\mathbf{2}}$

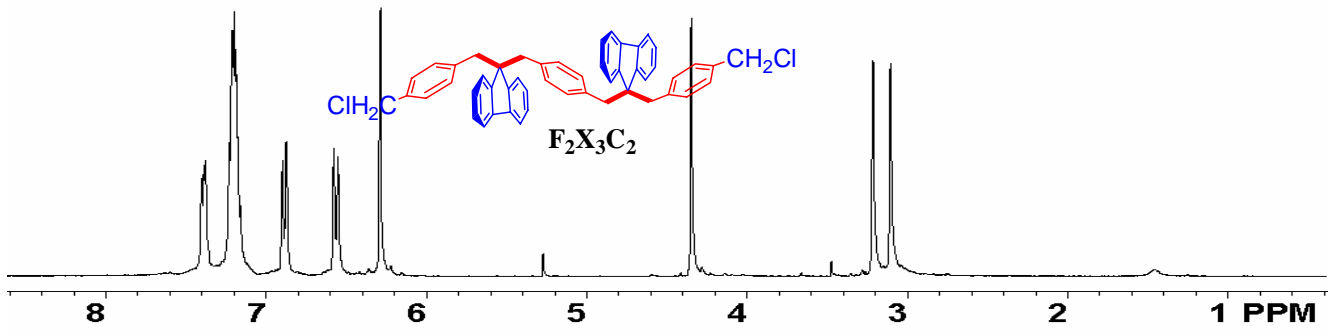




\section{${ }^{13} \mathrm{C}$ NMR spectrum of $\mathbf{F}_{\mathbf{2}} \mathbf{X}_{\mathbf{3}} \mathbf{C}_{\mathbf{2}}$}

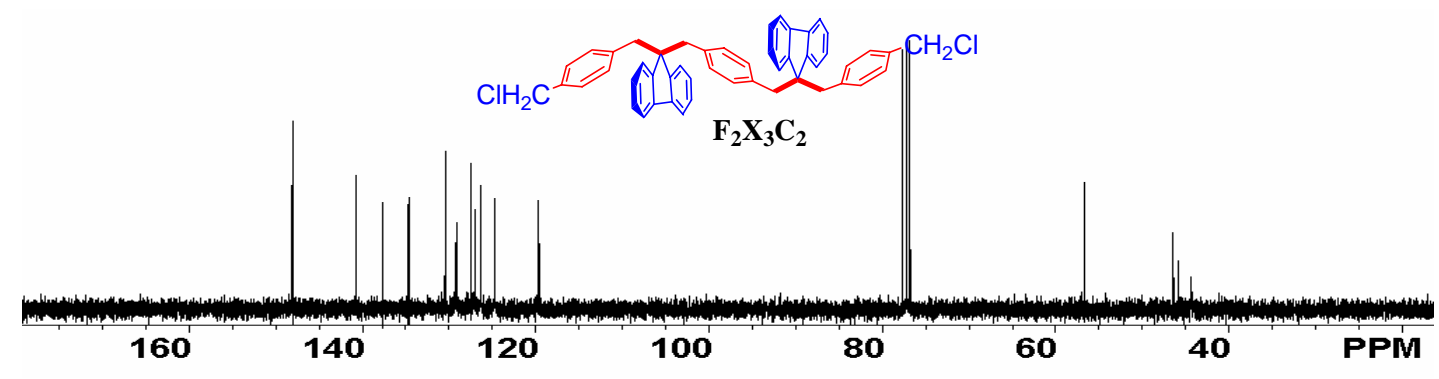


A

B
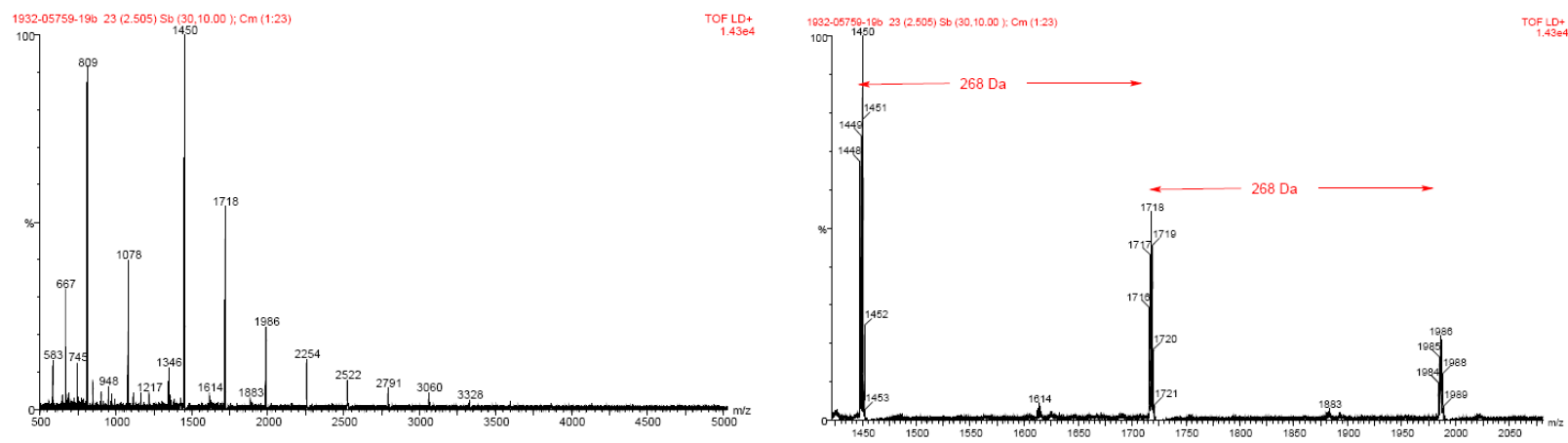

Figure S1. (A) Positive ion MALDI-TOF MS spectrum of mixtures of cyclic oligomers (structure B in Scheme 1) using Dithranol as the matrix and silver triflate as the cationization reagent. The spectrum represents the summation of scans from $>100$ laser shots collected by rastering various positions of the sample. (B) Expanded region of the positive ion MALDI-TOF MS spectrum showing that the polymeric distribution series' have a repeating unit of $268 \mathrm{Da}$. 


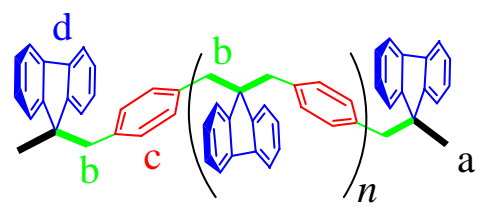

where $n=0-8$
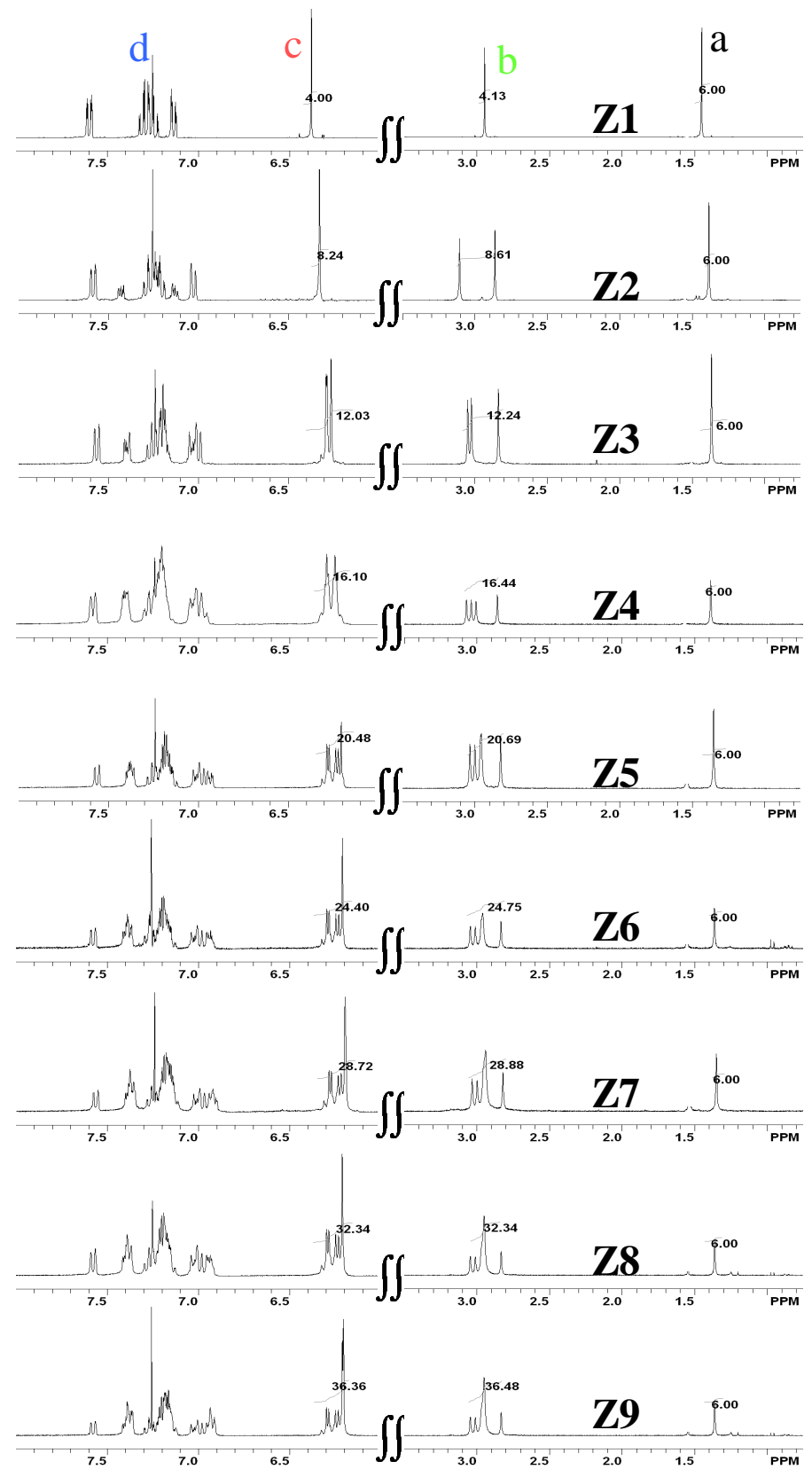

Figure S2. A comparison of the ${ }^{1} \mathrm{H}$ NMR spectra of $\mathbf{Z 1 - Z 9}$ oligomers. 

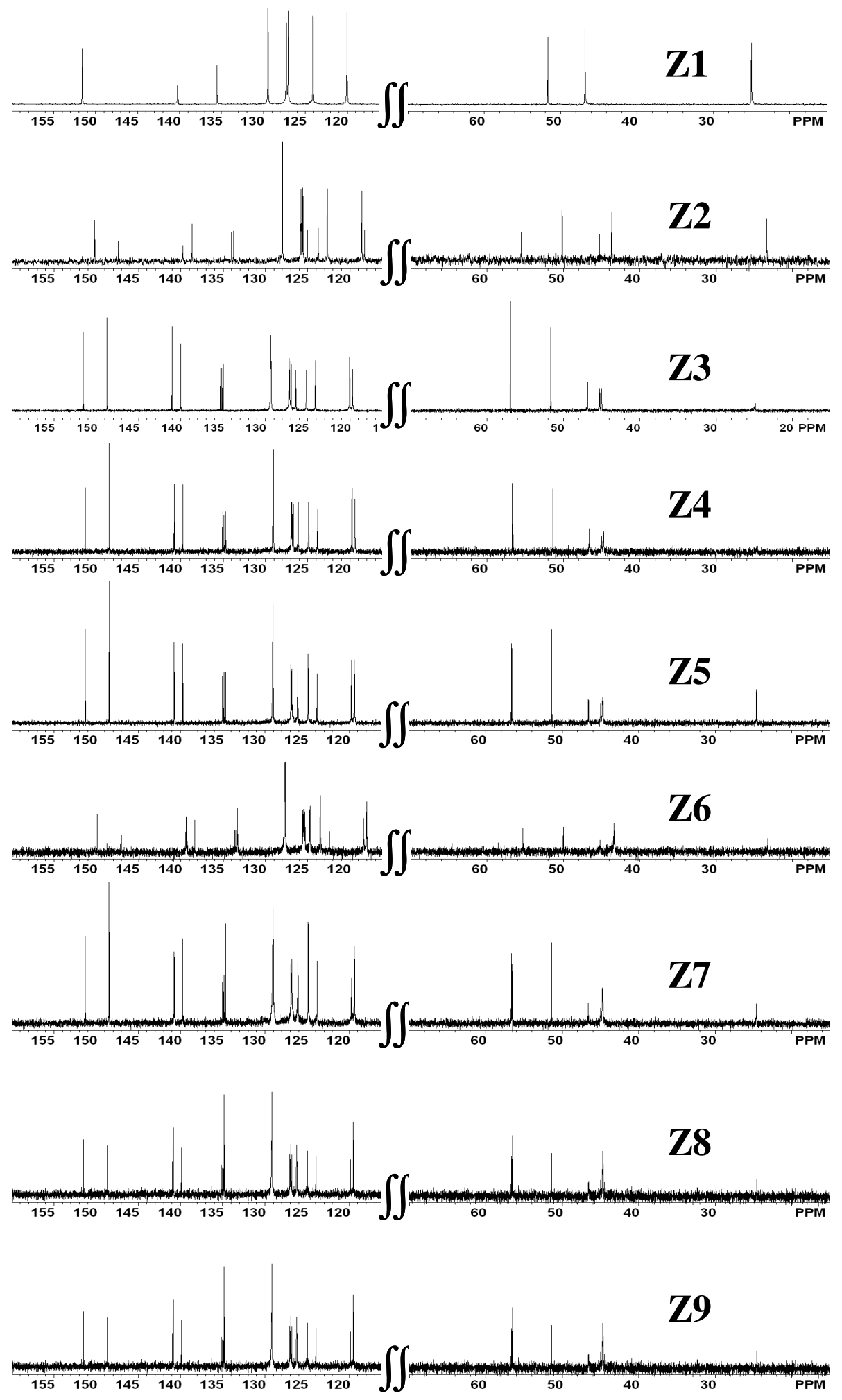

Figure S3. A comparison of the ${ }^{13} \mathrm{C}$ NMR spectra of Z1-Z9 oligomers. 


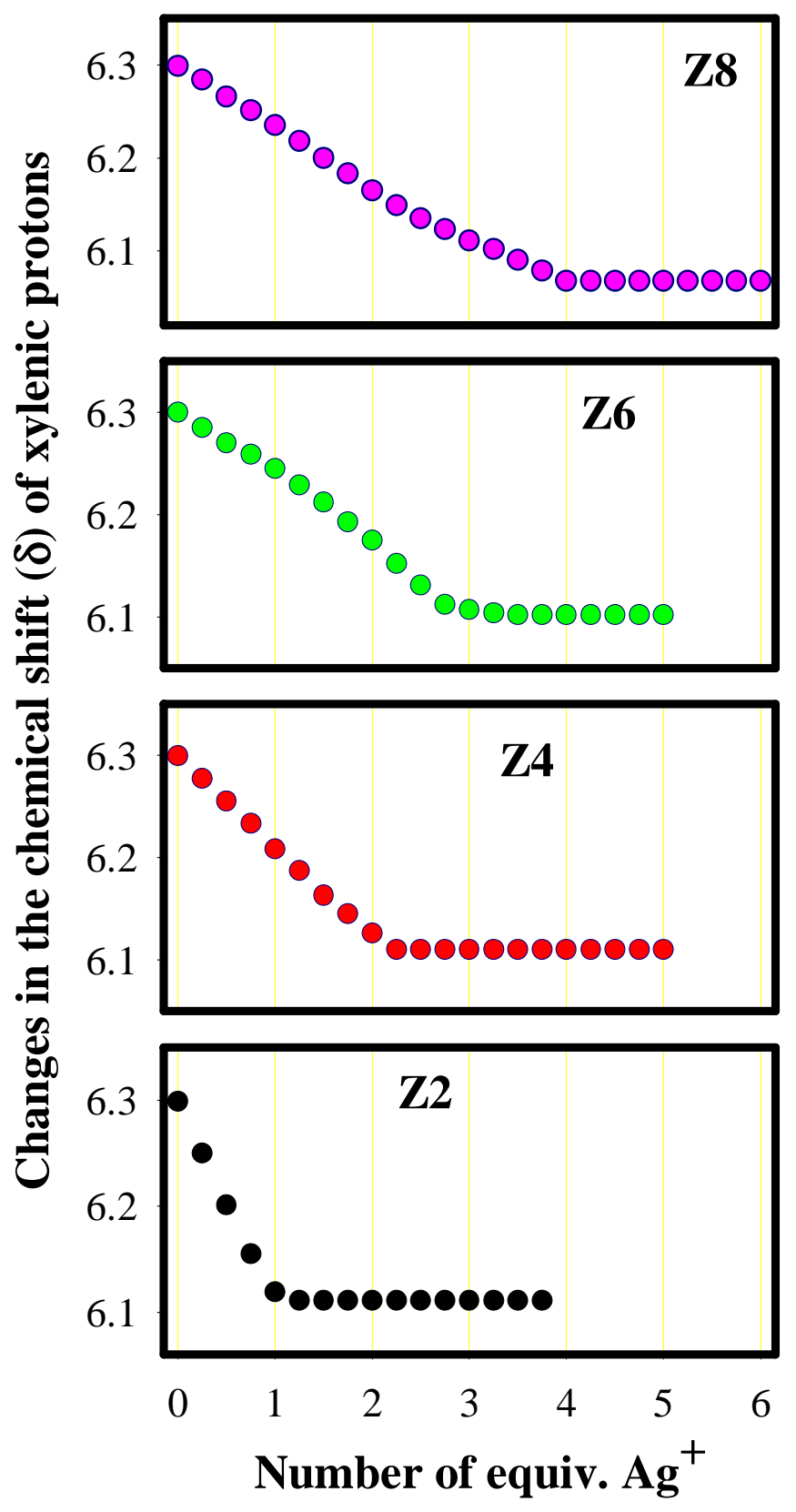

Figure S4. Plots of ${ }^{1} \mathrm{H}$ NMR chemical shift changes (in the xylenic protons) attendant upon the incremental addition of a $0.2-0.5 \mathrm{mM}$ solution of $\mathrm{Ag}^{+} \mathrm{CF}_{3} \mathrm{SO}_{3}{ }^{-}$ in 1:1 $\mathrm{CDCl}_{3} / \mathrm{CD}_{3} \mathrm{OD}$ to a $0.02-0.05 \mathrm{mM}$ solution of oligomers $\mathbf{Z 2}, \mathbf{Z 4}, \mathbf{Z 6}$, and $\mathbf{Z 8}$, containing odd numbers of fluoranyl moieties, in $\mathrm{CDCl}_{3} 22{ }^{\circ} \mathrm{C}$. For the actual ${ }^{1} \mathrm{H}$ NMR spectra obtained upon incremental addition of $\mathrm{Ag}^{+}$solution, see Figures S7, S9, S11, and S13 in the Supporting Information Section. 


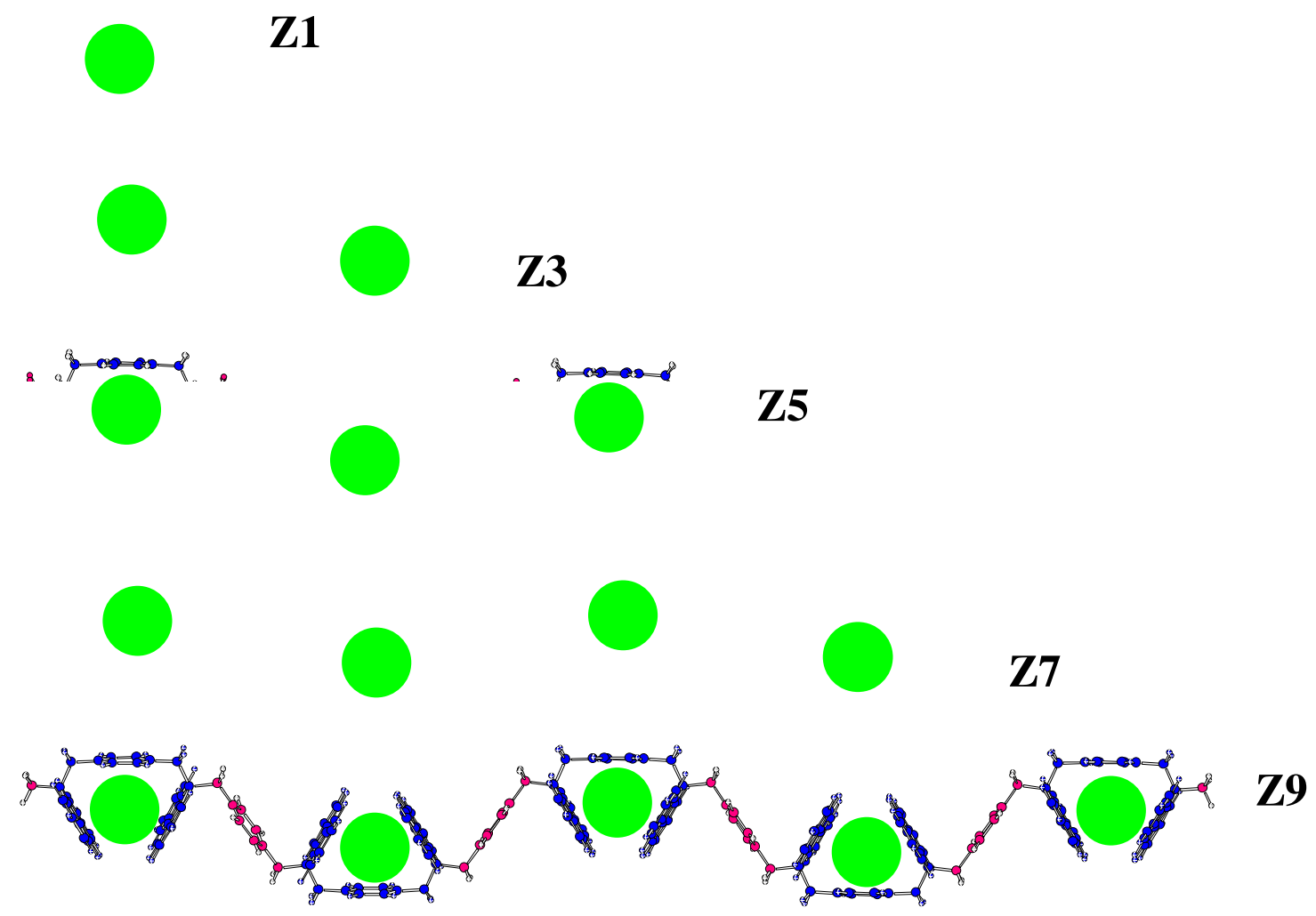

Figure S5. The calculated molecular structures of Z1, Z3, Z5, Z7, and Z9 using Spartan (AM1) showing the formation of multiple $\pi$-prismand-like cavities for the binding of $\mathrm{Ag}^{+}$cations. 

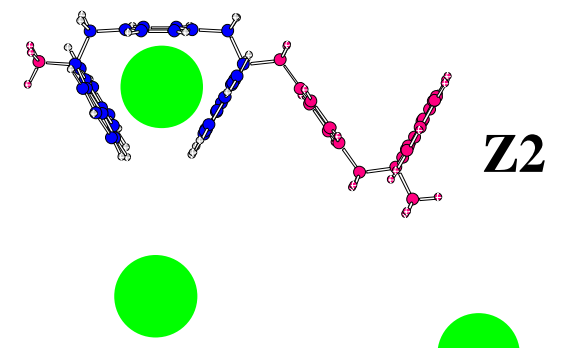

\section{$\mathbf{Z 4}$}
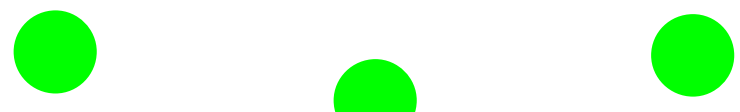

\section{Z6}

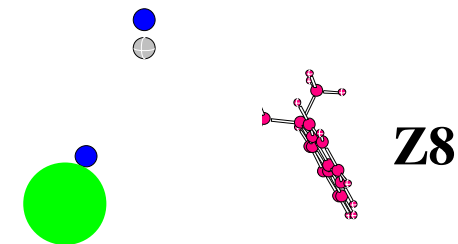

Figure S6. The calculated molecular structures of $\mathbf{Z 2}, \mathbf{Z 4}, \mathbf{Z 6}$, and $\mathbf{Z 8}$ using Spartan (AM1) showing the formation of multiple deltaphane-like cavities for the binding of $\mathrm{Ag}^{+}$cations as well as a single fluorene/xylene pair in an extended conformation. 
${ }^{1}$ H NMR Spectroscopic Titrations of Z1-Z9 with Silver Trifluoromethanesulfonate. The Stock solutions $(0.2-0.5 \mathrm{mM})$ of silver trifluoromethanesulfonate were prepared using $\mathrm{CDCl}_{3^{-}}$ $\mathrm{CD}_{3} \mathrm{OD}(1: 1, \mathrm{v} / \mathrm{v})$. Stock solutions of $\mathbf{Z 1 - Z 9}(0.02-0.05 \mathrm{mM})$ were prepared in $\mathrm{CDCl}_{3}$ in a medium wall NMR tube. ${ }^{1} \mathrm{H}$ NMR spectra were recorded after each addition of an appropriate aliquot of the stock solution of $\mathrm{Ag}^{+} \mathrm{CF}_{3} \mathrm{SO}_{3}{ }^{-}$, after vigorous shaking at $22{ }^{\circ} \mathrm{C}$. The spectra obtained for various oligomers are shown below in Figures S7-S14.

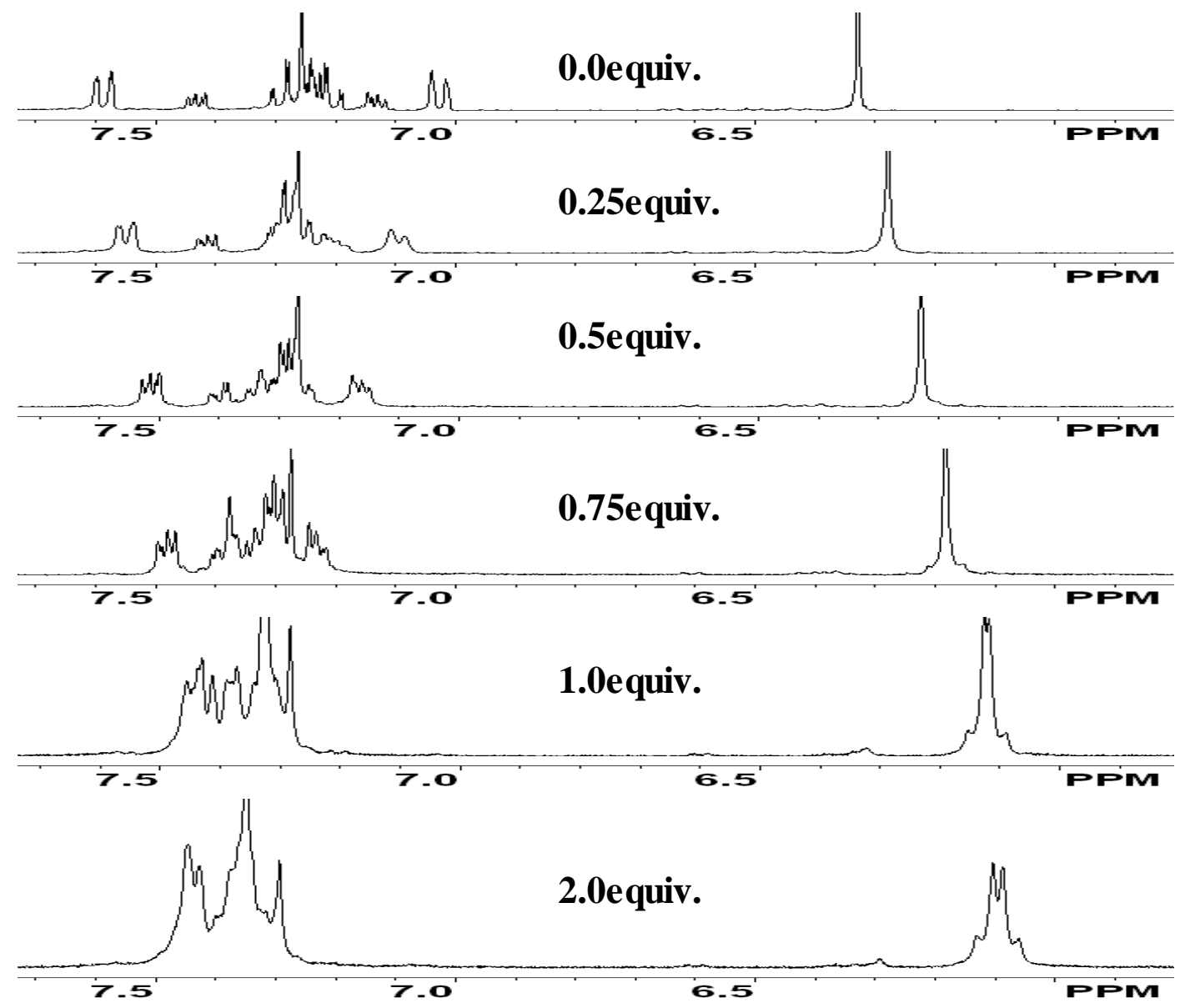

Figure S7. Partial ${ }^{1} \mathrm{H}$ NMR spectra of $\mathbf{Z 2}$ in $\mathrm{CDCl}_{3}(0.05 \mathrm{mM})$ obtained upon an incremental addition of $\mathrm{CF}_{3} \mathrm{SO}_{3} \mathrm{Ag}(0.5 \mathrm{mM})$ in $1: 1 \mathrm{CDCl}_{3}-\mathrm{CD}_{3} \mathrm{OD}$ at $22{ }^{\circ} \mathrm{C}$. 

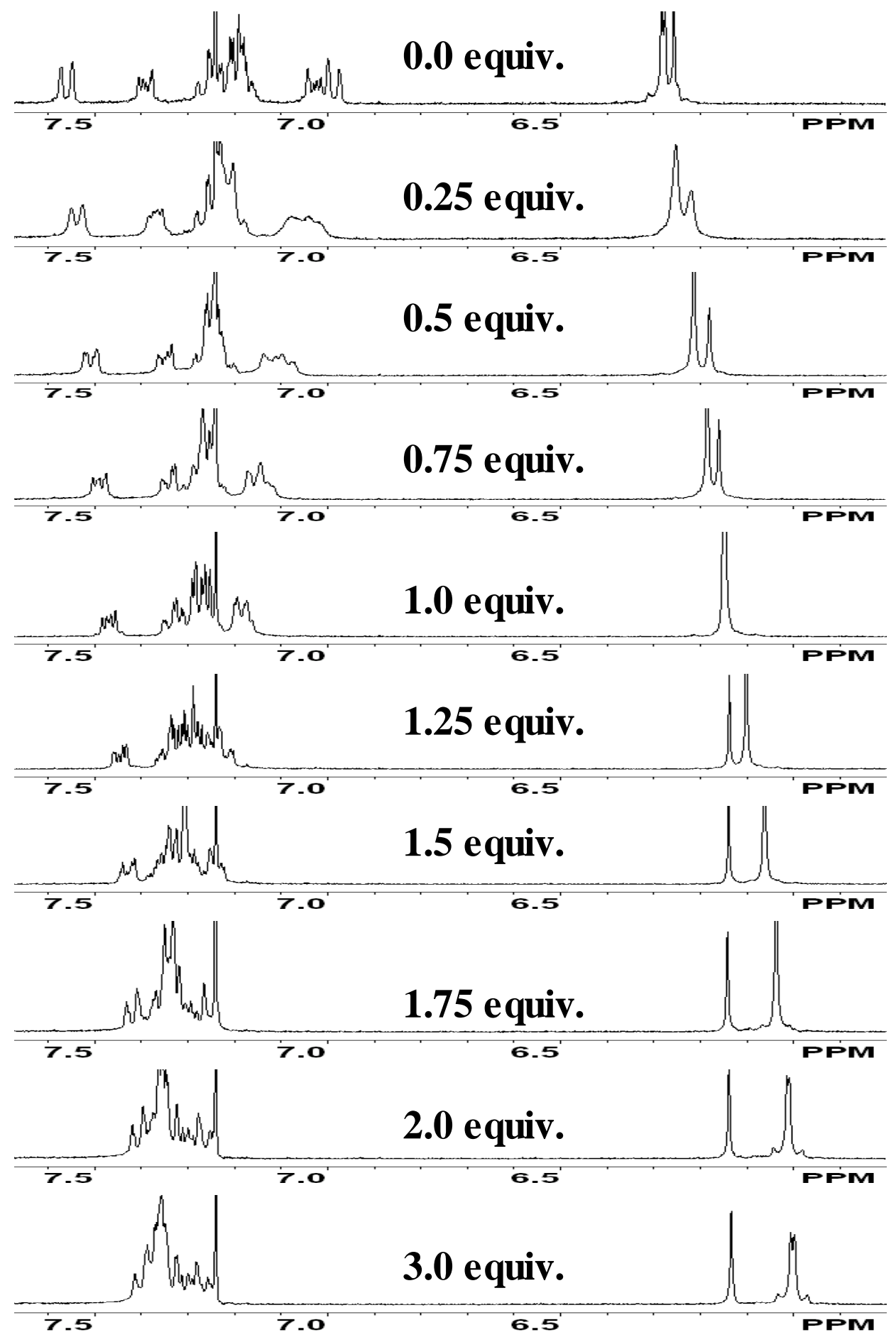

Figure S8. Partial ${ }^{1} \mathrm{H}$ NMR spectra of $\mathbf{Z 3}$ in $\mathrm{CDCl}_{3}(0.05 \mathrm{mM})$ obtained upon an incremental addition of $\mathrm{CF}_{3} \mathrm{SO}_{3} \mathrm{Ag}(0.5 \mathrm{mM})$ in $1: 1 \mathrm{CDCl}_{3}-\mathrm{CD}_{3} \mathrm{OD}$ at $22{ }^{\circ} \mathrm{C}$. 

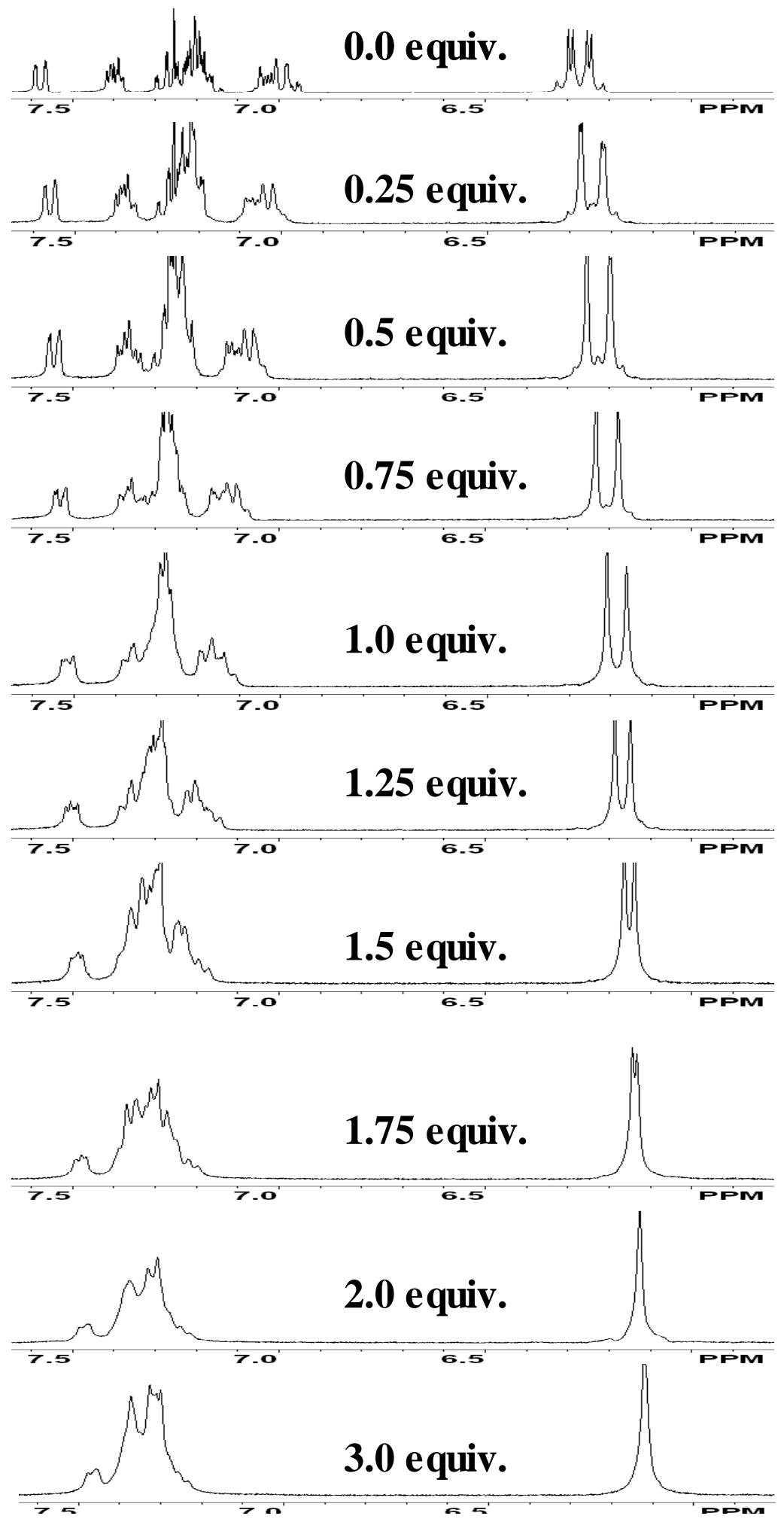

Figure S9. Partial ${ }^{1} \mathrm{H}$ NMR spectra of $\mathbf{Z 4}$ in $\mathrm{CDCl}_{3}(0.04 \mathrm{mM})$ obtained upon an incremental addition of $\mathrm{CF}_{3} \mathrm{SO}_{3} \mathrm{Ag}(0.4 \mathrm{mM})$ in $1: 1 \mathrm{CDCl}_{3}-\mathrm{CD}_{3} \mathrm{OD}$ at $22{ }^{\circ} \mathrm{C}$. 


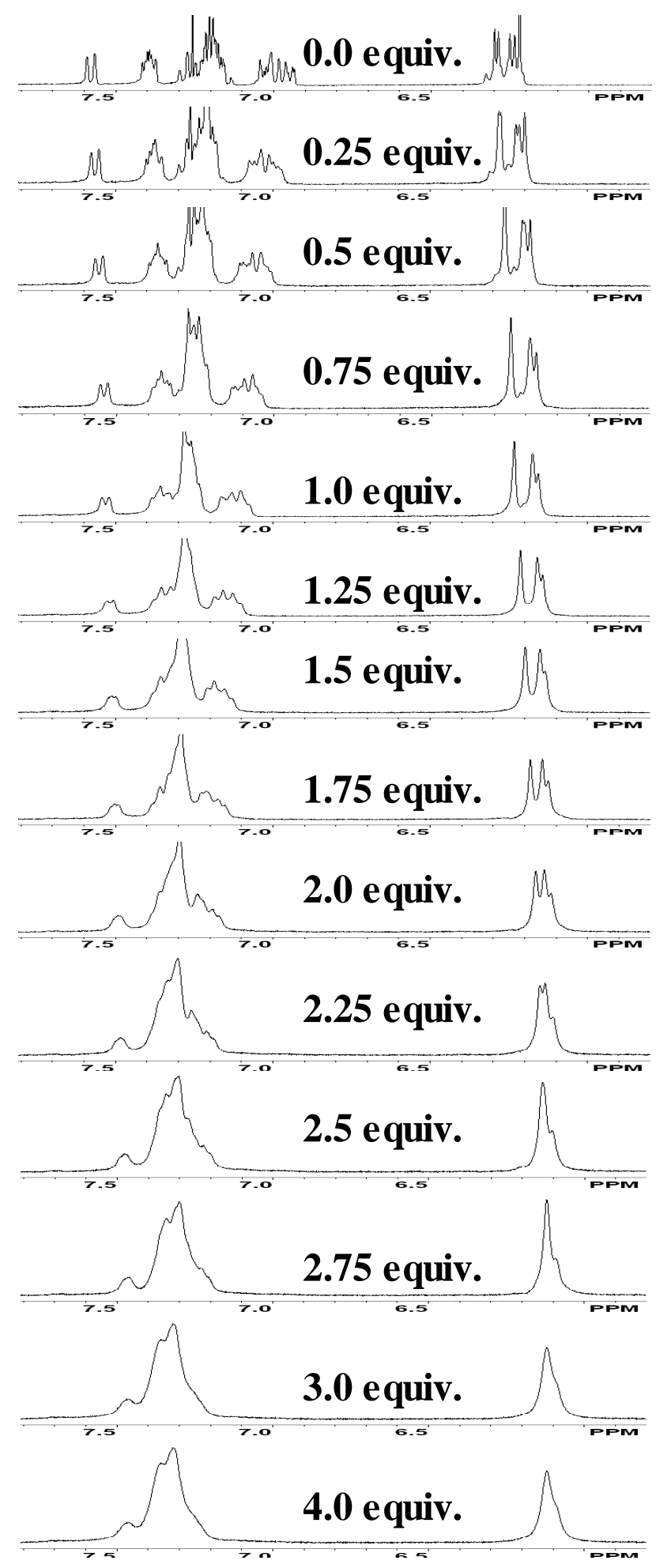

Figure S10. Partial ${ }^{1} \mathrm{H}$ NMR spectra of $\mathbf{Z 5}$ in $\mathrm{CDCl}_{3}(0.03 \mathrm{mM})$ obtained upon an incremental addition of $\mathrm{CF}_{3} \mathrm{SO}_{3} \mathrm{Ag}(0.3 \mathrm{mM})$ in $1: 1 \mathrm{CDCl}_{3}-\mathrm{CD}_{3} \mathrm{OD}$ at $22^{\circ} \mathrm{C}$. 

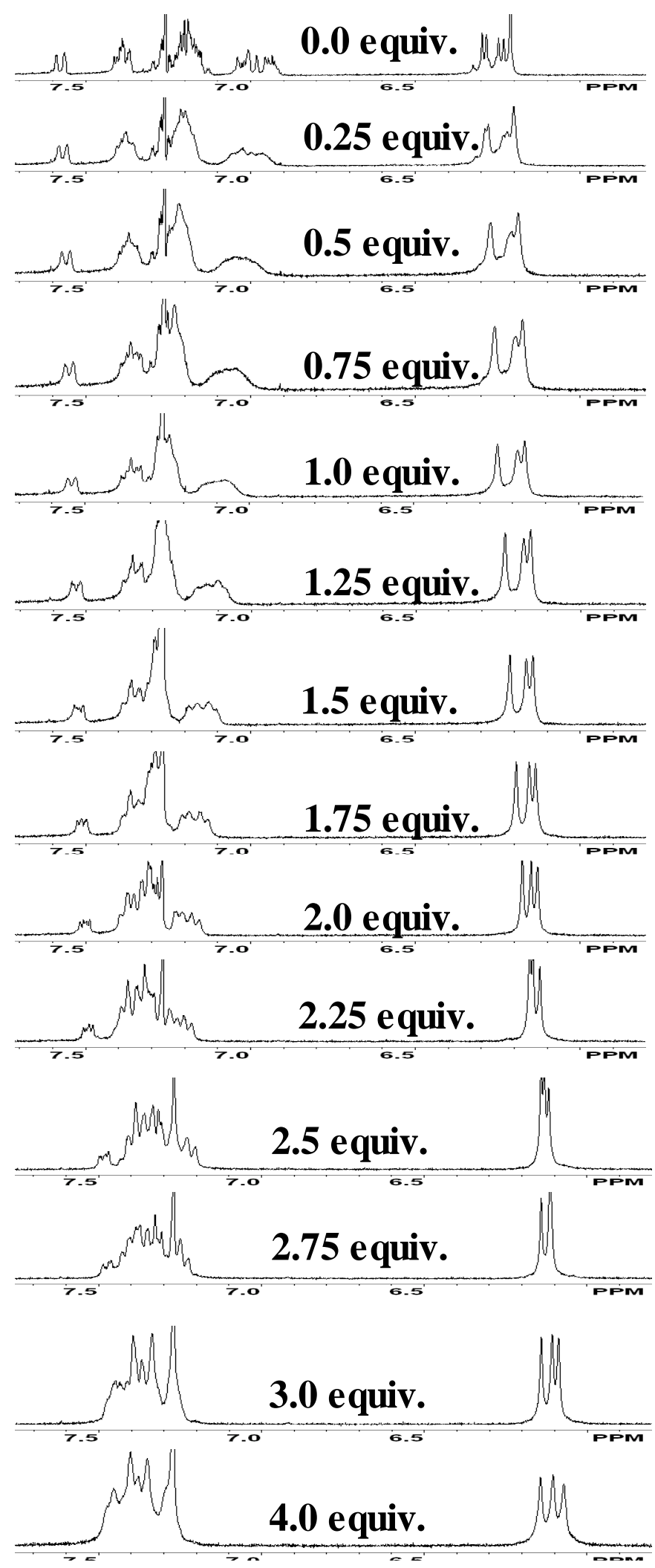

Figure S11. Partial ${ }^{1} \mathrm{H}$ NMR spectra of $\mathbf{Z 6}$ in $\mathrm{CDCl}_{3}(0.03 \mathrm{mM})$ obtained upon an incremental addition of $\mathrm{CF}_{3} \mathrm{SO}_{3} \mathrm{Ag}(0.3 \mathrm{mM})$ in $1: 1 \mathrm{CDCl}_{3}-\mathrm{CD}_{3} \mathrm{OD}$ at $22^{\circ} \mathrm{C}$. 


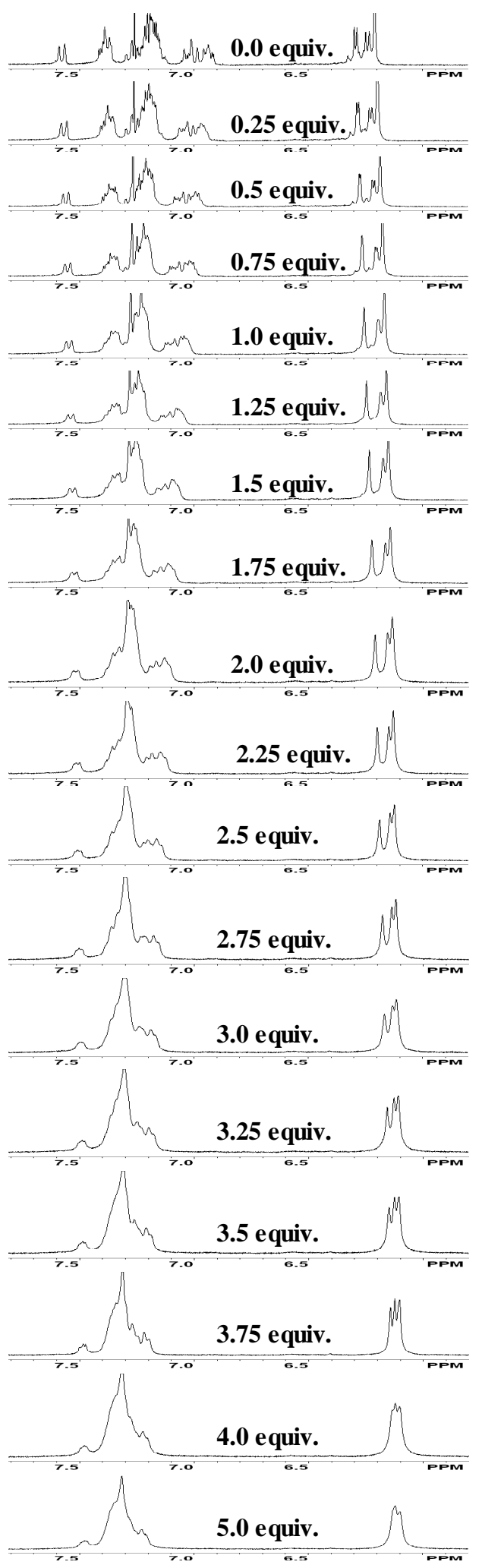

Figure S12. Partial ${ }^{1} \mathrm{H}$ NMR spectra of $\mathbf{Z 7}$ in $\mathrm{CDCl}_{3}(0.02 \mathrm{mM})$ obtained upon an incremental addition of $\mathrm{CF}_{3} \mathrm{SO}_{3} \mathrm{Ag}(0.2 \mathrm{mM})$ in $1: 1 \mathrm{CDCl}_{3}-\mathrm{CD}_{3} \mathrm{OD}$ at $22{ }^{\circ} \mathrm{C}$. 


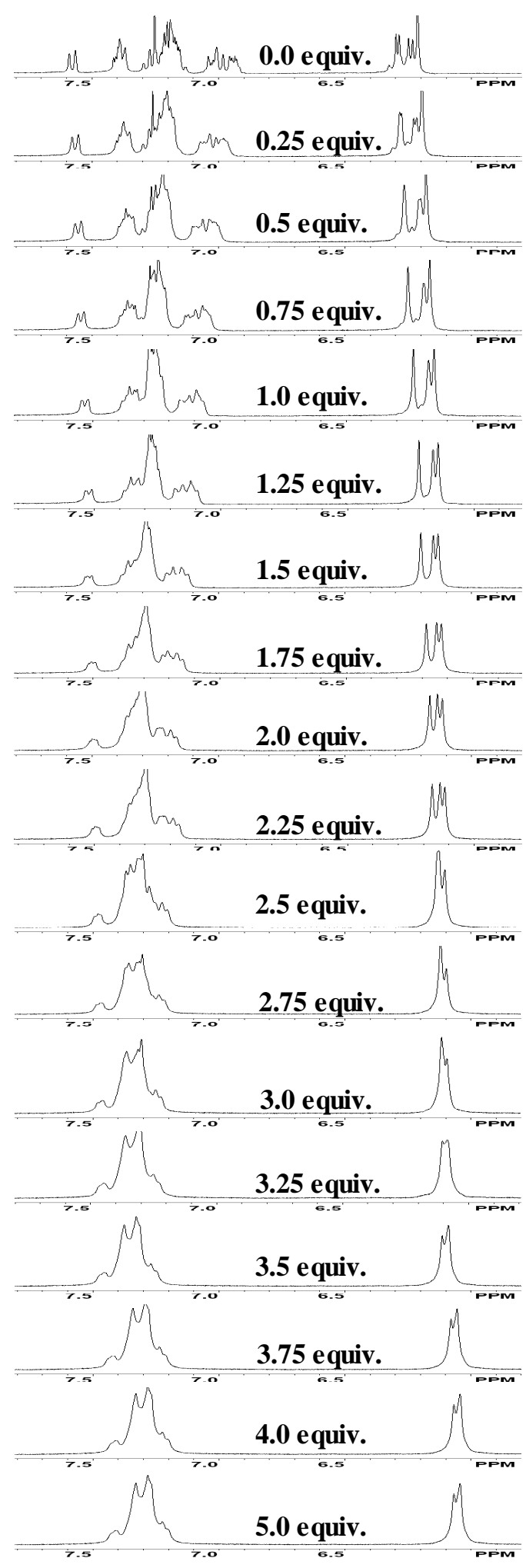

Figure S13. Partial ${ }^{1} \mathrm{H}$ NMR spectra of $\mathbf{Z 8}$ in $\mathrm{CDCl}_{3}(0.02 \mathrm{mM})$ obtained upon an incremental addition of $\mathrm{CF}_{3} \mathrm{SO}_{3} \mathrm{Ag}(0.2 \mathrm{mM})$ in $1: 1 \mathrm{CDCl}_{3}-\mathrm{CD}_{3} \mathrm{OD}$ at $22{ }^{\circ} \mathrm{C}$. 


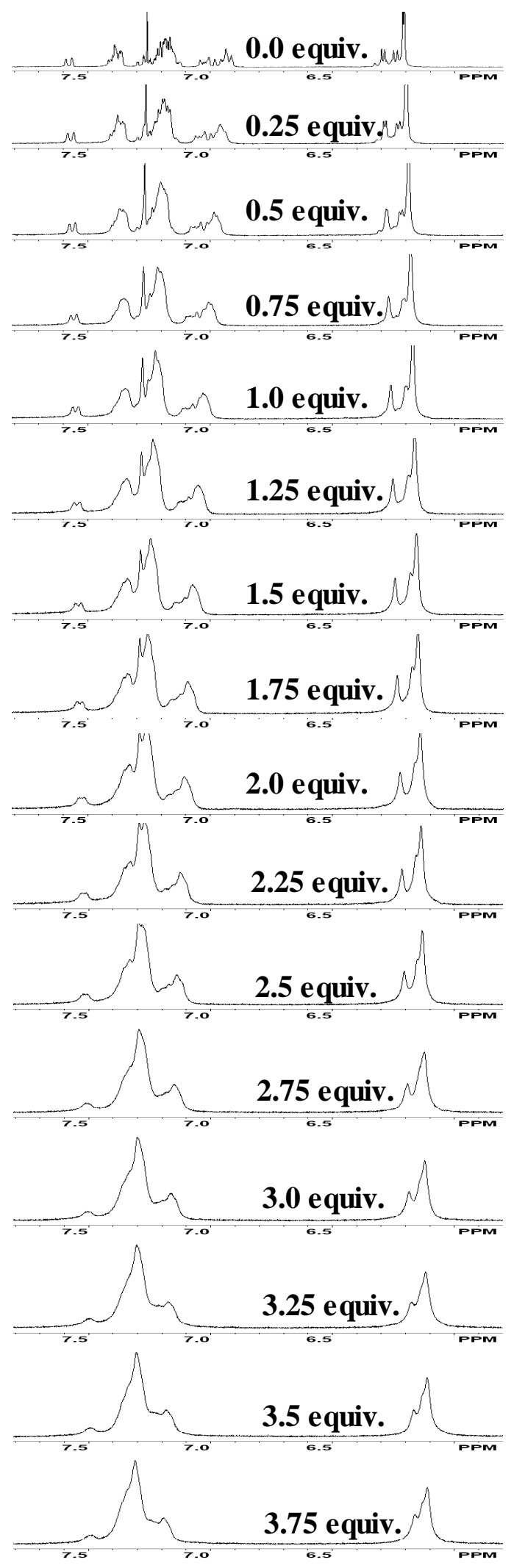

spectra continued on the next page........ 

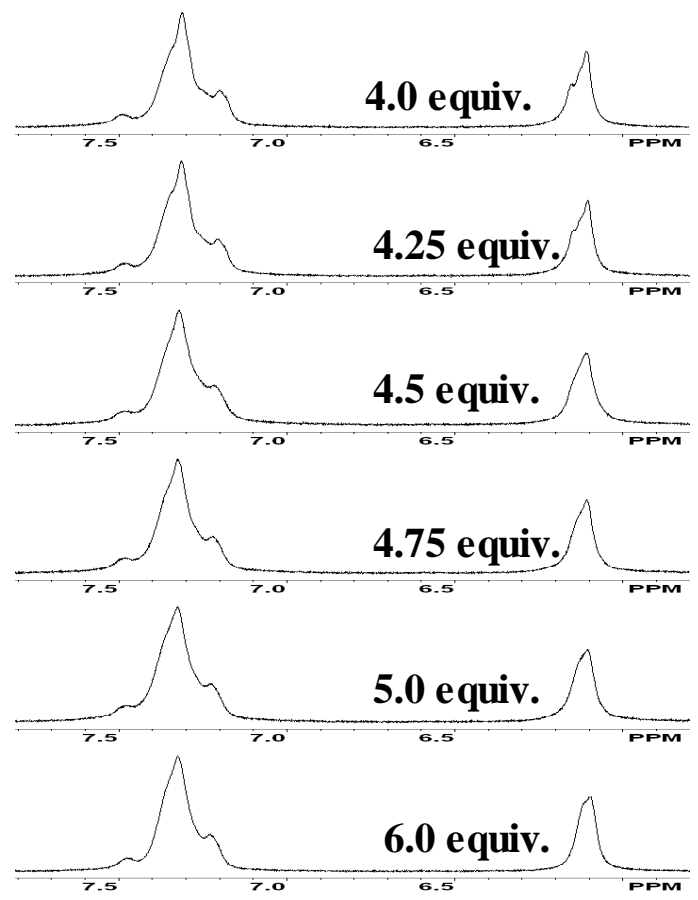

Figure S14. A plot of changes in the chemical shifts of the xylenic protons of $\mathbf{Z 9}$ in $\mathrm{CDCl}_{3}(0.02 \mathrm{mM})$ against the added equivalents of a solution of $\mathrm{CF}_{3} \mathrm{SO}_{3} \mathrm{Ag}$ $(0.2 \mathrm{mM})$ in $1: 1 \mathrm{CDCl}_{3}-\mathrm{CD}_{3} \mathrm{OD}$ at $22{ }^{\circ} \mathrm{C}$. 
Table 1. Crystal data and structure refinement for $\mathbf{Z 1}$ (raj03).

\begin{tabular}{|c|c|c|}
\hline Identification code & \multicolumn{2}{|l|}{ raj03 } \\
\hline Empirical formula & \multicolumn{2}{|l|}{$\mathrm{C}_{18} \mathrm{H}_{15}$} \\
\hline Formula weight & \multicolumn{2}{|l|}{231.30} \\
\hline Temperature & \multicolumn{2}{|l|}{$100(2) \mathrm{K}$} \\
\hline Wavelength & \multicolumn{2}{|l|}{$0.71073 \AA$} \\
\hline Crystal system & \multicolumn{2}{|l|}{ Monoclinic } \\
\hline Space group & \multicolumn{2}{|l|}{$\mathrm{P} 2{ }_{1} / \mathrm{c}$} \\
\hline \multirow[t]{3}{*}{ Unit cell dimensions } & $\mathrm{a}=8.7700(6) \AA$ & $\alpha=90^{\circ}$ \\
\hline & $\mathrm{b}=20.3186(13) \AA$ & $\beta=110.552(1)^{\circ}$. \\
\hline & $c=7.8056(5) \AA$ & $\gamma=90^{\circ}$ \\
\hline Volume & \multicolumn{2}{|l|}{$1302.39(15) \AA^{3}$} \\
\hline $\mathrm{Z}$ & \multicolumn{2}{|l|}{4} \\
\hline Density (calculated) & \multicolumn{2}{|l|}{$1.180 \mathrm{Mg} / \mathrm{m}^{3}$} \\
\hline Absorption coefficient & \multicolumn{2}{|l|}{$0.066 \mathrm{~mm}^{-1}$} \\
\hline $\mathrm{F}(000)$ & \multicolumn{2}{|l|}{492} \\
\hline Crystal size & \multicolumn{2}{|c|}{$0.50 \times 0.43 \times 0.32 \mathrm{~mm}^{3}$} \\
\hline Theta range for data collection & \multicolumn{2}{|l|}{2.00 to $26.37^{\circ}$} \\
\hline Index ranges & \multicolumn{2}{|c|}{$-10<=\mathrm{h}<=10,-25<=\mathrm{k}<=25,-9<=1<=9$} \\
\hline Reflections collected & \multicolumn{2}{|c|}{10595} \\
\hline Independent reflections & \multicolumn{2}{|c|}{$2658[\mathrm{R}($ int $)=0.0302]$} \\
\hline Completeness to theta $=26.37^{\circ}$ & \multicolumn{2}{|l|}{$99.9 \%$} \\
\hline Absorption correction & \multicolumn{2}{|c|}{ Empirical with SADABS } \\
\hline Max. and min. transmission & \multicolumn{2}{|l|}{0.9790 and 0.9675} \\
\hline Refinement method & \multicolumn{2}{|c|}{ Full-matrix least-squares on $\mathrm{F}^{2}$} \\
\hline Data / restraints / parameters & \multicolumn{2}{|l|}{$2658 / 0 / 164$} \\
\hline Goodness-of-fit on $\mathrm{F}^{2}$ & \multicolumn{2}{|l|}{1.012} \\
\hline Final $R$ indices $[\mathrm{I}>2 \operatorname{sigma}(\mathrm{I})]$ & \multicolumn{2}{|c|}{$\mathrm{R} 1=0.0404, \mathrm{wR} 2=0.0974$} \\
\hline $\mathrm{R}$ indices (all data) & \multicolumn{2}{|c|}{$\mathrm{R} 1=0.0454, \mathrm{wR} 2=0.1009$} \\
\hline Largest diff. peak and hole & \multicolumn{2}{|c|}{0.279 and -0.161 e. $\AA^{-3}$} \\
\hline
\end{tabular}

Table 2. Atomic coordinates ( $\left.\mathrm{x} 10^{4}\right)$ and equivalent isotropic displacement parameters $\left(\AA^{2} \times 10^{3}\right)$ for raj03. $U(e q)$ is defined as one third of the trace of the orthogonalized $U^{i j}$ tensor.

\begin{tabular}{|c|c|c|c|c|}
\hline & $\mathrm{X}$ & $\mathrm{y}$ & $\mathrm{z}$ & $\mathrm{U}(\mathrm{eq})$ \\
\hline$\overline{C(1)}$ & $2202(1)$ & $6269(1)$ & $2732(2)$ & $23(1)$ \\
\hline $\mathrm{C}(2)$ & $3835(2)$ & $6116(1)$ & $3199(2)$ & $29(1)$ \\
\hline $\mathrm{C}(3)$ & $4317(2)$ & $5716(1)$ & $2030(2)$ & $35(1)$ \\
\hline $\mathrm{C}(4)$ & $3174(2)$ & $5464(1)$ & $442(2)$ & $36(1)$ \\
\hline $\mathrm{C}(5)$ & $1535(2)$ & $5616(1)$ & $-38(2)$ & $31(1)$ \\
\hline $\mathrm{C}(6)$ & 1059(1) & $6030(1)$ & $1101(2)$ & $24(1)$ \\
\hline $\mathrm{C}(7)$ & $-540(1)$ & $6312(1)$ & $893(2)$ & $25(1)$ \\
\hline $\mathrm{C}(8)$ & $-2052(2)$ & $6241(1)$ & $-489(2)$ & $34(1)$ \\
\hline $\mathrm{C}(9)$ & $-3364(2)$ & $6590(1)$ & $-345(2)$ & $43(1)$ \\
\hline $\mathrm{C}(10)$ & $-3172(2)$ & $7002(1)$ & $1135(2)$ & $44(1)$ \\
\hline $\mathrm{C}(11)$ & $-1668(2)$ & $7070(1)$ & $2525(2)$ & $36(1)$ \\
\hline$C(12)$ & $-356(1)$ & $6721(1)$ & $2397(2)$ & $26(1)$ \\
\hline $\mathrm{C}(13)$ & $1375(1)$ & $6686(1)$ & $3768(2)$ & $24(1)$ \\
\hline $\mathrm{C}(14)$ & 2171(2) & $7364(1)$ & $4314(2)$ & $31(1)$ \\
\hline$C(15)$ & $1381(2)$ & $6326(1)$ & $5541(2)$ & $27(1)$ \\
\hline$C(16)$ & $662(2)$ & $5641(1)$ & $5245(2)$ & $25(1)$ \\
\hline
\end{tabular}




\begin{tabular}{lllll}
$\mathrm{C}(17)$ & $-976(2)$ & $5536(1)$ & $4973(2)$ & $27(1)$ \\
$\mathrm{C}(18)$ & $1625(2)$ & $5095(1)$ & $5262(2)$ & $27(1)$ \\
\hline
\end{tabular}

Table 3. Bond lengths $[\AA]$ and angles $\left[{ }^{\circ}\right]$ for raj03.

\begin{tabular}{|c|c|c|c|}
\hline$C(1)-C(2)$ & $1.3834(16)$ & $C(9)-C(10)$ & $1.388(2)$ \\
\hline$C(1)-C(6)$ & $1.4021(16)$ & $C(10)-C(11)$ & $1.390(2)$ \\
\hline$C(1)-C(13)$ & $1.5202(16)$ & $\mathrm{C}(11)-\mathrm{C}(12)$ & $1.3852(17)$ \\
\hline$C(2)-C(3)$ & $1.3937(19)$ & $\mathrm{C}(12)-\mathrm{C}(13)$ & $1.5209(16)$ \\
\hline$C(3)-C(4)$ & $1.390(2)$ & $C(13)-C(14)$ & $1.5345(16)$ \\
\hline$C(4)-C(5)$ & $1.3868(19)$ & $C(13)-C(15)$ & $1.5640(15)$ \\
\hline$C(5)-C(6)$ & $1.3904(17)$ & $\mathrm{C}(15)-\mathrm{C}(16)$ & $1.5116(16)$ \\
\hline$C(6)-C(7)$ & $1.4700(16)$ & $C(16)-C(18)$ & $1.3921(17)$ \\
\hline$C(7)-C(8)$ & $1.3919(17)$ & $C(16)-C(17)$ & $1.3928(17)$ \\
\hline$C(7)-C(12)$ & $1.4014(17)$ & $\mathrm{C}(17)-\mathrm{C}(18) \# 1$ & $1.3881(17)$ \\
\hline $\mathrm{C}(8)-\mathrm{C}(9)$ & $1.389(2)$ & $\mathrm{C}(18)-\mathrm{C}(17) \# 1$ & $1.3881(17)$ \\
\hline$C(2)-C(1)-C(6)$ & $120.34(11)$ & & \\
\hline $\mathrm{C}(2)-\mathrm{C}(1)-\mathrm{C}(13)$ & $128.94(11)$ & & \\
\hline$C(6)-C(1)-C(13)$ & $110.72(10)$ & & \\
\hline$C(1)-C(2)-C(3)$ & $118.87(12)$ & & \\
\hline$C(4)-C(3)-C(2)$ & $120.60(12)$ & & \\
\hline$C(5)-C(4)-C(3)$ & 120.91(12) & & \\
\hline$C(4)-C(5)-C(6)$ & $118.52(12)$ & & \\
\hline$C(5)-C(6)-C(1)$ & $120.72(11)$ & & \\
\hline$C(5)-C(6)-C(7)$ & $130.84(11)$ & & \\
\hline$C(1)-C(6)-C(7)$ & $108.39(10)$ & & \\
\hline$C(8)-C(7)-C(12)$ & $120.56(11)$ & & \\
\hline$C(8)-C(7)-C(6)$ & 131.15(12) & & \\
\hline$C(12)-C(7)-C(6)$ & $108.28(10)$ & & \\
\hline$C(9)-C(8)-C(7)$ & 118.41(13) & & \\
\hline $\mathrm{C}(10)-\mathrm{C}(9)-\mathrm{C}(8)$ & $120.87(13)$ & & \\
\hline $\mathrm{C}(9)-\mathrm{C}(10)-\mathrm{C}(11)$ & 120.96(13) & & \\
\hline $\mathrm{C}(12)-\mathrm{C}(11)-\mathrm{C}(10)$ & $118.50(13)$ & & \\
\hline$C(11)-C(12)-C(7)$ & $120.68(12)$ & & \\
\hline $\mathrm{C}(11)-\mathrm{C}(12)-\mathrm{C}(13)$ & $128.47(12)$ & & \\
\hline $\mathrm{C}(7)-\mathrm{C}(12)-\mathrm{C}(13)$ & $110.80(10)$ & & \\
\hline$C(1)-C(13)-C(12)$ & $101.32(9)$ & & \\
\hline $\mathrm{C}(1)-\mathrm{C}(13)-\mathrm{C}(14)$ & 113.09(10) & & \\
\hline $\mathrm{C}(12)-\mathrm{C}(13)-\mathrm{C}(14)$ & 113.51(10) & & \\
\hline $\mathrm{C}(1)-\mathrm{C}(13)-\mathrm{C}(15)$ & 110.83(9) & & \\
\hline $\mathrm{C}(12)-\mathrm{C}(13)-\mathrm{C}(15)$ & $109.66(9)$ & & \\
\hline $\mathrm{C}(14)-\mathrm{C}(13)-\mathrm{C}(15)$ & $108.31(9)$ & & \\
\hline $\mathrm{C}(16)-\mathrm{C}(15)-\mathrm{C}(13)$ & $115.09(9)$ & & \\
\hline $\mathrm{C}(18)-\mathrm{C}(16)-\mathrm{C}(17)$ & $117.92(11)$ & & \\
\hline $\mathrm{C}(18)-\mathrm{C}(16)-\mathrm{C}(15)$ & 120.91(11) & & \\
\hline $\mathrm{C}(17)-\mathrm{C}(16)-\mathrm{C}(15)$ & 121.16(11) & & \\
\hline $\mathrm{C}(18) \# 1-\mathrm{C}(17)-\mathrm{C}(16)$ & 121.03(11) & & \\
\hline $\mathrm{C}(17) \# 1-\mathrm{C}(18)-\mathrm{C}(16)$ & 121.04(11) & & \\
\hline
\end{tabular}


Symmetry transformations used to generate equivalent atoms: \#1 -x,-y+1,-z+1

Table 4. Anisotropic displacement parameters $\left(\AA^{2} \times 10^{3}\right)$ for raj03. The anisotropic displacement factor exponent takes the form: $-2 \pi^{2}\left[h^{2} a^{* 2} U^{11}+\ldots+2 h k a^{*} b^{*} U^{12}\right]$

\begin{tabular}{lcccccc}
\hline & $\mathrm{U}^{11}$ & $\mathrm{U}^{22}$ & $\mathrm{U}^{33}$ & $\mathrm{U}^{23}$ & $\mathrm{U}^{13}$ & $\mathrm{U}^{12}$ \\
\hline $\mathrm{C}(1)$ & $27(1)$ & $19(1)$ & $25(1)$ & $4(1)$ & $10(1)$ & $-1(1)$ \\
$\mathrm{C}(2)$ & $26(1)$ & $29(1)$ & $31(1)$ & $4(1)$ & $8(1)$ & $0(1)$ \\
$\mathrm{C}(3)$ & $30(1)$ & $35(1)$ & $45(1)$ & $9(1)$ & $19(1)$ & $8(1)$ \\
$\mathrm{C}(4)$ & $45(1)$ & $32(1)$ & $39(1)$ & $-1(1)$ & $25(1)$ & $5(1)$ \\
$\mathrm{C}(5)$ & $39(1)$ & $29(1)$ & $26(1)$ & $-1(1)$ & $14(1)$ & $-3(1)$ \\
$\mathrm{C}(6)$ & $28(1)$ & $21(1)$ & $24(1)$ & $4(1)$ & $10(1)$ & $-2(1)$ \\
$\mathrm{C}(7)$ & $26(1)$ & $22(1)$ & $28(1)$ & $6(1)$ & $9(1)$ & $-3(1)$ \\
$\mathrm{C}(8)$ & $30(1)$ & $34(1)$ & $34(1)$ & $8(1)$ & $5(1)$ & $-6(1)$ \\
$\mathrm{C}(9)$ & $24(1)$ & $46(1)$ & $51(1)$ & $16(1)$ & $3(1)$ & $-3(1)$ \\
$\mathrm{C}(10)$ & $28(1)$ & $41(1)$ & $67(1)$ & $15(1)$ & $20(1)$ & $9(1)$ \\
$\mathrm{C}(11)$ & $35(1)$ & $29(1)$ & $49(1)$ & $6(1)$ & $21(1)$ & $6(1)$ \\
$\mathrm{C}(12)$ & $27(1)$ & $21(1)$ & $31(1)$ & $5(1)$ & $12(1)$ & $0(1)$ \\
$\mathrm{C}(13)$ & $27(1)$ & $21(1)$ & $26(1)$ & $0(1)$ & $10(1)$ & $-1(1)$ \\
$\mathrm{C}(14)$ & $39(1)$ & $23(1)$ & $32(1)$ & $-1(1)$ & $13(1)$ & $-5(1)$ \\
$\mathrm{C}(15)$ & $33(1)$ & $25(1)$ & $24(1)$ & $-1(1)$ & $10(1)$ & $-4(1)$ \\
$\mathrm{C}(16)$ & $32(1)$ & $24(1)$ & $17(1)$ & $1(1)$ & $8(1)$ & $-4(1)$ \\
$\mathrm{C}(17)$ & $32(1)$ & $24(1)$ & $27(1)$ & $2(1)$ & $12(1)$ & $2(1)$ \\
$\mathrm{C}(18)$ & $27(1)$ & $29(1)$ & $25(1)$ & $3(1)$ & $9(1)$ & $-2(1)$ \\
& & & & & \\
\hline
\end{tabular}

Table 5. Hydrogen coordinates ( $\left.\mathrm{x} 10^{4}\right)$ and isotropic displacement parameters $\left(\AA^{2} \times 10^{3}\right)$ for raj03.

\begin{tabular}{lrrrr}
\hline & $\mathrm{x}$ & $\mathrm{y}$ & $\mathrm{z}$ & $\mathrm{U}(\mathrm{eq})$ \\
\hline & & & & \\
$\mathrm{H}(2)$ & 4614 & 6281 & 4297 & 35 \\
$\mathrm{H}(3)$ & 5437 & 5614 & 2323 & 42 \\
$\mathrm{H}(4)$ & 3520 & 5184 & -325 & 43 \\
$\mathrm{H}(5)$ & 756 & 5441 & -1122 & 37 \\
$\mathrm{H}(8)$ & -2183 & 5962 & -1507 & 41 \\
$\mathrm{H}(9)$ & -4406 & 6546 & -1272 & 51 \\
$\mathrm{H}(10)$ & -4082 & 7241 & 1199 & 53 \\
$\mathrm{H}(11)$ & -1542 & 7351 & 3540 & 43 \\
$\mathrm{H}(14 \mathrm{~A})$ & 3256 & 7309 & 5246 & 46 \\
$\mathrm{H}(14 \mathrm{~B})$ & 1497 & 7634 & 4808 & 46 \\
$\mathrm{H}(14 \mathrm{C})$ & 2270 & 7581 & 3236 & 46 \\
$\mathrm{H}(15 \mathrm{~A})$ & 2519 & 6298 & 6402 & 33 \\
$\mathrm{H}(15 \mathrm{~B})$ & 766 & 6597 & 6130 & 33 \\
$\mathrm{H}(17)$ & -1659 & 5901 & 4949 & 33 \\
$\mathrm{H}(18)$ & 2744 & 5154 & 5437 & 32 \\
\end{tabular}


Table 6. Torsion angles $\left[{ }^{\circ}\right]$ for raj03.

\begin{tabular}{|c|c|}
\hline $\mathrm{C}(6)-\mathrm{C}(1)-\mathrm{C}(2)-\mathrm{C}(3)$ & $-0.56(17)$ \\
\hline $\mathrm{C}(13)-\mathrm{C}(1)-\mathrm{C}(2)-\mathrm{C}(3)$ & $179.50(11)$ \\
\hline $\mathrm{C}(1)-\mathrm{C}(2)-\mathrm{C}(3)-\mathrm{C}(4)$ & $-1.27(19)$ \\
\hline $\mathrm{C}(2)-\mathrm{C}(3)-\mathrm{C}(4)-\mathrm{C}(5)$ & $1.4(2)$ \\
\hline$C(3)-C(4)-C(5)-C(6)$ & $0.32(19)$ \\
\hline$C(4)-C(5)-C(6)-C(1)$ & $-2.16(17)$ \\
\hline$C(4)-C(5)-C(6)-C(7)$ & $175.13(12)$ \\
\hline $\mathrm{C}(2)-\mathrm{C}(1)-\mathrm{C}(6)-\mathrm{C}(5)$ & $2.31(17)$ \\
\hline$C(13)-C(1)-C(6)-C(5)$ & $-177.74(10)$ \\
\hline$C(2)-C(1)-C(6)-C(7)$ & $-175.53(10)$ \\
\hline$C(13)-C(1)-C(6)-C(7)$ & $4.42(12)$ \\
\hline$C(5)-C(6)-C(7)-C(8)$ & $1.1(2)$ \\
\hline $\mathrm{C}(1)-\mathrm{C}(6)-\mathrm{C}(7)-\mathrm{C}(8)$ & $178.60(12)$ \\
\hline$C(5)-C(6)-C(7)-C(12)$ & $-177.51(12)$ \\
\hline $\mathrm{C}(1)-\mathrm{C}(6)-\mathrm{C}(7)-\mathrm{C}(12)$ & $0.04(12)$ \\
\hline $\mathrm{C}(12)-\mathrm{C}(7)-\mathrm{C}(8)-\mathrm{C}(9)$ & $0.48(18)$ \\
\hline $\mathrm{C}(6)-\mathrm{C}(7)-\mathrm{C}(8)-\mathrm{C}(9)$ & $-177.94(12)$ \\
\hline $\mathrm{C}(7)-\mathrm{C}(8)-\mathrm{C}(9)-\mathrm{C}(10)$ & $0.4(2)$ \\
\hline $\mathrm{C}(8)-\mathrm{C}(9)-\mathrm{C}(10)-\mathrm{C}(11)$ & $-0.9(2)$ \\
\hline $\mathrm{C}(9)-\mathrm{C}(10)-\mathrm{C}(11)-\mathrm{C}(12)$ & $0.4(2)$ \\
\hline $\mathrm{C}(10)-\mathrm{C}(11)-\mathrm{C}(12)-\mathrm{C}(7)$ & $0.51(18)$ \\
\hline $\mathrm{C}(10)-\mathrm{C}(11)-\mathrm{C}(12)-\mathrm{C}(13)$ & $-176.78(12)$ \\
\hline $\mathrm{C}(8)-\mathrm{C}(7)-\mathrm{C}(12)-\mathrm{C}(11)$ & $-0.96(17)$ \\
\hline $\mathrm{C}(6)-\mathrm{C}(7)-\mathrm{C}(12)-\mathrm{C}(11)$ & $177.79(11)$ \\
\hline $\mathrm{C}(8)-\mathrm{C}(7)-\mathrm{C}(12)-\mathrm{C}(13)$ & 176.77(10) \\
\hline $\mathrm{C}(6)-\mathrm{C}(7)-\mathrm{C}(12)-\mathrm{C}(13)$ & $-4.48(12)$ \\
\hline$C(2)-C(1)-C(13)-C(12)$ & $173.29(11)$ \\
\hline$C(6)-C(1)-C(13)-C(12)$ & $-6.65(11)$ \\
\hline $\mathrm{C}(2)-\mathrm{C}(1)-\mathrm{C}(13)-\mathrm{C}(14)$ & $51.44(16)$ \\
\hline $\mathrm{C}(6)-\mathrm{C}(1)-\mathrm{C}(13)-\mathrm{C}(14)$ & $-128.50(10)$ \\
\hline$C(2)-C(1)-C(13)-C(15)$ & $-70.40(15)$ \\
\hline$C(6)-C(1)-C(13)-C(15)$ & $109.65(11)$ \\
\hline $\mathrm{C}(11)-\mathrm{C}(12)-\mathrm{C}(13)-\mathrm{C}(1)$ & $-175.80(12)$ \\
\hline $\mathrm{C}(7)-\mathrm{C}(12)-\mathrm{C}(13)-\mathrm{C}(1)$ & $6.69(12)$ \\
\hline $\mathrm{C}(11)-\mathrm{C}(12)-\mathrm{C}(13)-\mathrm{C}(14)$ & $-54.25(16)$ \\
\hline $\mathrm{C}(7)-\mathrm{C}(12)-\mathrm{C}(13)-\mathrm{C}(14)$ & $128.24(11)$ \\
\hline $\mathrm{C}(11)-\mathrm{C}(12)-\mathrm{C}(13)-\mathrm{C}(15)$ & $67.03(15)$ \\
\hline $\mathrm{C}(7)-\mathrm{C}(12)-\mathrm{C}(13)-\mathrm{C}(15)$ & $-110.48(11)$ \\
\hline $\mathrm{C}(1)-\mathrm{C}(13)-\mathrm{C}(15)-\mathrm{C}(16)$ & $-54.28(13)$ \\
\hline $\mathrm{C}(12)-\mathrm{C}(13)-\mathrm{C}(15)-\mathrm{C}(16)$ & $56.75(13)$ \\
\hline$C(14)-C(13)-C(15)-C(16)$ & $-178.89(10)$ \\
\hline $\mathrm{C}(13)-\mathrm{C}(15)-\mathrm{C}(16)-\mathrm{C}(18)$ & $88.36(13)$ \\
\hline $\mathrm{C}(13)-\mathrm{C}(15)-\mathrm{C}(16)-\mathrm{C}(17)$ & $-92.58(13)$ \\
\hline $\mathrm{C}(18)-\mathrm{C}(16)-\mathrm{C}(17)-\mathrm{C}(18) \# 1$ & $0.38(18)$ \\
\hline $\mathrm{C}(15)-\mathrm{C}(16)-\mathrm{C}(17)-\mathrm{C}(18) \# 1$ & $-178.71(11)$ \\
\hline $\mathrm{C}(17)-\mathrm{C}(16)-\mathrm{C}(18)-\mathrm{C}(17) \# 1$ & $-0.38(18)$ \\
\hline $\mathrm{C}(15)-\mathrm{C}(16)-\mathrm{C}(18)-\mathrm{C}(17) \# 1$ & 178.71(10) \\
\hline
\end{tabular}

Symmetry transformations used to generate equivalent atoms:

$\# 1-\mathrm{x},-\mathrm{y}+1,-\mathrm{z}+1$ 